\title{
Scoping review of chronic rhinosinusitis proteomics*
}

\section{Stephen Shih-Teng Kao', Ahmed Bassiouni', Mahnaz Ramezanpour', Nusha Chegeni², Alex D. Colella², Timothy K. Chataway², Peter-John Wormald', Sarah Vreugde', Alkis James Psaltis ${ }^{1}$}

Rhinology 58: 5, 418 - 470, 2020

https://doi.org/10.4193/Rhin20.034

*Received for publication:

February 2, 2020

Accepted: March 29, 2020

\begin{abstract}
Background: Progressive advances in proteomic technology has improved our understanding of the chronic rhinosinusitis (CRS) pathogenesis and endotypes. This scoping review aims to present a comprehensive and descriptive analysis of nasal mucosa and mucus proteome of CRS patients.
\end{abstract}

Methodology: Studies investigating the proteome of nasal mucosa and mucus from healthy and CRS patients via mass spectrometry were included. Critical appraisal of methodological quality was conducted with extraction of protein lists. Gene set enrichment analysis (GSEA) was performed on studies including CRS patients.

Results: 2962 proteins were identified in the 21 studies included in this review. Eleven studies investigated the nasal mucus proteome and ten studies investigated the nasal mucosa proteome. Studies demonstrated heterogeneity in patients, sampling and mass spectrometry methodology. Samples from CRS patients suggested a trend in enrichment of immune system and programmed cell death pathways. Increased expression of proteins involved in cellular components including the cytoskeleton and adherens junctions was also present in CRS.

Conclusions: Alterations in the healthy sinonasal proteome may lead to the increased immunological, metabolic and tissue remodeling processes observed in CRS. However, it is difficult to draw significant conclusions from the GSEA due to the heterogeneity present in the limited literature available. These findings allow us to direct further research to better understand CRS pathogenesis and its endotypes.

Key words: chronic rhinosinusitis, mucus, proteomics, scoping review, mass spectrometry

\section{Introduction}

Chronic rhinosinusitis (CRS) is characterised by persisting inflammation of the mucosa in the nasal cavity and paranasal sinuses. The current phenotypic classification of CRS is divided into CRS without nasal polyps (CRSsNP) or CRS with nasal polyps (CRSwNP). These phenotypes, however, do not cover the diverse cellular pathways involved in the complex pathogenesis of CRS. Advances in laboratory techniques has led to investigating CRS endotypes, enabling a better understanding of upstream pathogenic factors that lead to CRS disease manifestation and recalcitrance. Ultimately, CRS endotypes may allow clinicians to predict disease prognosis and develop personalised treatment regimes.

The mucosal barrier, in concert with an effective mucociliary transport system and a protective mucus layer interact together with the innate and humoral immune system to maintain homeostasis in health. Tight junctions located on the apical aspects of cell membranes inhibit the flow of solutes and water into the paracellular space in addition to forming cell polarity $^{(1-3)}$. Studies have demonstrated down regulation of tight junction transmembrane and associated proteins with reduced transmembrane electrical resistance in patients with CRS (4-6). Furthermore various inflammatory cytokines including IL-17, 
IL-33, TLSP have been implicated in the pathogenesis of CRS and nasal polyp formation ${ }^{(7-10)}$. The heterogeneity of immune responses in CRS endotypes certainly plays a significant role in the manifestation of the disease.

Nasal mucus, a vital component of the mucociliary clearance system, is produced by seromucinous glands, goblet cells and the transudation of plasma. It is composed of glycoproteins and polysaccharide chains organised into two layers; the lower periciliary layer and the superficial viscous layer ${ }^{(11,12)}$. The antimicrobial effects of nasal mucus have been well studied ${ }^{(11,12)}$. The overproduction of viscous nasal mucus is commonly reported amongst CRS patients manifested as rhinorrhoea and postnasal drip. This tenacious mucus adversely affects ciliary function, leading to stasis that may result in a nidus for bacterial growth ${ }^{(13-15)}$.

The proteomic analysis of nasal mucus and mucosa is a rapidly evolving field exploring the physiological and pathological mechanisms involved in CRS. In this form of analysis, mucus and tissue samples collected from patients undergo gel electrophoresis or liquid chromatography to separate the proteins ${ }^{(16)}$. Proteins are subjected to mass spectrometry (MS) for identification of peptide sequences, which are compared against known protein databases. Previous studies have provided insight into the proteins involved in various immune responses from mucus and mucosa collected from CRS patients ${ }^{(16-18)}$. The aim of this scoping review was to perform a comprehensive review of the current literature on the CRS proteome and to ascertain differences between CRS and healthy patients. Ultimately, improving the knowledge of CRS endotypes through cellular pathways present will guide prognostication and treatment regimens for CRS patients.

\section{Methods}

The search strategy aimed to capture all English language studies published in Pubmed, CINAHL, Embase and Cochrane CENTRAL databases since their creation. A preliminary initial search of the Pubmed database using the keywords "Chronic rhinosinusitis", "Mucus", and "Proteomics" was initially performed to identify further relevant key words to be included in the final search strategy of all databases (Supplement 1). A second search using all identified keywords and medical subject headings were applied across all databases. This review adhered to the PRISMA guidelines for reporting systematic reviews. The reference lists of all selected studies were searched for additional studies.

Studies conducting proteomic analysis on patients 18 years or older via mass spectrometry on mucus samples or nasal mucosa collected from healthy or CRS patients were included. Studies conducting bottom up proteomics were included, with studies investigating select proteins were excluded. Patients diagnosed with acute sinusitis or allergic rhinitis or exposure to chemicals or fumes were excluded. Studies utilizing tissue collected from the olfactory bulb, olfactory epithelium, nerve tissue, or tissue grown in vitro were excluded. Proteins mentioned in systemic reviews, meta-analyses, literature reviews and conferences abstracts were excluded to prevent duplication of data.

Papers retrieved that met all inclusion criteria were assessed by two independent reviewers (SSK, AB) for methodological validity prior to inclusion in the review. Differences in assessments between reviewers were resolved through discussion with a third reviewer (TC).

Data extraction focused on proteins identified through mass spectrometry in healthy and CRS patients. Key data extracted included disease status (Healthy or CRS), tissue collection method, tissue specimen examined (Mucus or Mucosa), analytical platform and protein composition. Proteins identified were stratified by mucus or mucosa origin and disease status.

\section{Statistical analysis}

Statistical analysis was conducted with R statistical software (R Core team, Vienna, Austria) ${ }^{(19)}$. All proteins identified from studies were matched manually with their Uniprot accession numbers, protein name and gene. Proteins not in the Uniprot database or not from Homo Sapiens genus were excluded from the analysis. Proteins were stratified into their presence in either CRS, healthy groups or both for mucus and mucosa samples. Mucus and mucosa protein lists were processed separately, due to their differing constituents. Gene set enrichment analysis (GSEA) was conducted, clustering the available differentially expressed genes for analysis. This was performed by uploading protein lists to Enrichr' ${ }^{\mathrm{TM}}$ (Ma'ayan Laboratory, USA), a web-based software ${ }^{(20,21)}$. Reactome 2016 pathway database identified cellular pathways, and the Gene Ontology database identified biological processes, cellular components and molecular functions. Only significant results (Adjusted P-value $<0.05$ calculated by Enrichr $^{\mathrm{TM}}$ ) were included for further analysis. Mucus and mucosa GSEA results were combined for analysis. No further analysis was possible due to the lack of available numeric quantifiable data from the studies included in the review.

The cellular pathways generated from CRS mucus and mucosa were then combined. This was also performed for the biological processes, cellular components and molecular functions. The same was performed for proteins unique to the healthy group, and proteins present in both healthy and CRS groups.

\section{Results}

The search identified a total of 591 studies in English, with 526 remaining after duplicates were removed. Titles and abstracts 
Table 1. Studies investigating proteomics of nasal mucus.

\begin{tabular}{|c|c|c|c|c|c|c|}
\hline Author & $\begin{array}{l}\text { Disease } \\
\text { Status }\end{array}$ & Patients & $\begin{array}{l}\text { Collection } \\
\text { Method }\end{array}$ & MS Method & MS Equipment & $\begin{array}{l}\text { Proteins } \\
\text { Identified }\end{array}$ \\
\hline $\begin{array}{l}\text { Benson } \\
\text { et al. }{ }^{38}\end{array}$ & CRSsNP & 6 & NLF & Nano-LC-MS/MS & $\begin{array}{l}\text { LC (Michrom BioResources Inc; CA, USA) } \\
\text { LTQ Linear ion trap MS (ThermoFinnigan; CA, USA) }\end{array}$ & 129 \\
\hline $\begin{array}{l}\text { Casado } \\
\text { et al. }{ }^{39}\end{array}$ & Healthy & 10 & NLF & $\begin{array}{l}\text { Micro-Capillary LC } \\
\text { ESI-Q-TOF MS }\end{array}$ & $\begin{array}{l}\text { CapLC (Waters; Milford, USA) } \\
\text { ESI-Q-TOF MS (Micromass; Manchester, UK) }\end{array}$ & 111 \\
\hline $\begin{array}{l}\text { Debat } \\
\text { et al. }{ }^{26}\end{array}$ & Healthy & 16 & $\begin{array}{l}\text { Suction } \\
\text { (Olfactory } \\
\text { cleft) }\end{array}$ & MALDI-TOF-MS & Voyager DE STR+ TOF-MS (Applied Biosystems; CA, USA) & 75 \\
\hline $\begin{array}{l}\text { Ghafouri } \\
\text { et al. }{ }^{33}\end{array}$ & Healthy & 5 & NLF & $\begin{array}{l}\text { MALDI-TOF MS } \\
\text { Nanoelectrospray } \\
\text { MS/MS }\end{array}$ & $\begin{array}{l}\text { Voyager DE PRO MS (Applied Biosystems; CA, USA) } \\
\text { API Q-STAR Pulzer i (Applied biosystems; CA, USA) with } \\
\text { nanoelectrospray ion source (MDS-Protana; Odense, } \\
\text { Denmark) }\end{array}$ & 20 \\
\hline $\begin{array}{l}\text { Lindahl } \\
\text { et al. }{ }^{34}\end{array}$ & Healthy & 7 & NLF & MALDI-TOF-MS & $\begin{array}{l}\text { Voyager DE STR MS (PE-Biosystems; CA, USA) or } \\
\text { Bruker Reflex (Bruker Daltonics; Bremen, Germany) }\end{array}$ & 12 \\
\hline $\begin{array}{l}\text { Mortstedt } \\
\text { et al. }{ }^{35}\end{array}$ & Healthy & 8 & NLF & $\begin{array}{l}\text { Micro-LC-MS/MS } \\
\text { Nano-LC-Q-TOF MS }\end{array}$ & $\begin{array}{l}\text { LC (UFLCXR, Shimadzu Corporation; Kyoto, Japan) } \\
\text { QTRAP } 5500 \text { hybrid triple quadrupole/ linear ion trap MS } \\
\text { (Applied Biosystems/ MDS Sciex; MA, USA) } \\
\text { LC (Agilent } 1100 \text { series; CA, USA) } \\
\text { QSTAR pulsar hybrid quadrupole TOF MS (Applied Biosys- } \\
\text { tems/MDS Sciex; MA, USA) }\end{array}$ & 331 \\
\hline $\begin{array}{l}\text { Schoenebeck } \\
\text { et al. }{ }^{27}\end{array}$ & Healthy & N/A & NLF & $\begin{array}{l}\text { Nano-LC-MS/MS } \\
\text { Micro-LC-MS/MS }\end{array}$ & $\begin{array}{l}\text { LC (Ultimate HPLC system, Dionex; Idstein, Germany) } \\
\text { HCT ultra PTM analysis system (Bruker Daltonic; Bremen, } \\
\text { Germany) } \\
\text { LC (HP Ultimate } 300 \text { system, Dionex; Idstein, Germany) } \\
\text { LTQ OrbiTrap Velos MS (Thermofisher Scientific; MA, USA) }\end{array}$ & 34 \\
\hline $\begin{array}{l}\text { Tewfik } \\
\text { et al. }{ }^{16}\end{array}$ & $\begin{array}{l}\text { Healthy } \\
\text { CRSwNP }\end{array}$ & $\begin{array}{l}4 \\
4\end{array}$ & Suction & Micro-LC-MS/MS & $\begin{array}{l}\text { LC (Agilent Technologies; Ontario, Canada) } \\
\text { QTRAP } 4000 \text { (Sciex-Applied Biosystems; Ontario, Canada) }\end{array}$ & 35 \\
\hline $\begin{array}{l}\text { Tomazic } \\
\text { et al. }{ }^{22}\end{array}$ & Healthy & 29 & $\begin{array}{l}\text { Suction } \\
\text { (Middle } \\
\text { meatus) }\end{array}$ & Nano-LC-MS/MS & $\begin{array}{l}\text { Nano-HPLC (Agilent } 1200 \text { series; Vienna, Austria) } \\
\text { LTQ-FT MS (Thermo Scientific; Vienna, Austria) }\end{array}$ & 247 \\
\hline $\begin{array}{l}\text { Tomazic } \\
\text { et al. }\end{array}$ & Healthy & 12 & $\begin{array}{l}\text { Suction } \\
\text { (Middle } \\
\text { meatus) }\end{array}$ & Nano-LC-MS/MS & $\begin{array}{l}\text { Nano-HPLC (Agilent } 1200 \text { series; Vienna, Austria) } \\
\text { LTQ-FT MS (Thermo Scientific; Vienna, Austria) }\end{array}$ & 366 \\
\hline $\begin{array}{l}\text { Wahlen } \\
\text { et al. } .^{37}\end{array}$ & Healthy & 13 & NLF & MALDI-TOF MS & Voyager DE PRO (Applied Biosystems; CA, USA) & 48 \\
\hline
\end{tabular}

HPLC: High performance liquid chromatography; LC: Liquid Chromatography; NLF: Nasal lavage fluid; MS: Mass spectrometry; LC-MS/MS: Liquid chromatography-tandem mass spectrometry; ESI-Q-TOF: Electrospray-ionisation quadrupole time-of-flight mass spectrometry; LC-Q-TOF: Liquid chromatography/ quadrupole time-of-flight mass spectrometry; MALDI-TOF MS: Matrix Assisted Laser Desorption/ lonisation mass spectrometry.

were reviewed against the inclusion criteria yielding a total of 52 studies for full text analysis. 21 studies met the inclusion criteria (Supplement 2).

Studies included were published between 2004 to 2018 originating from Austria ${ }^{(22,23)}$, Canada ${ }^{(16)}$, China $^{(24)}$, Finland ${ }^{(25)}$, France ${ }^{(26)}$, Germany $^{(18,27)}$, Italy ${ }^{(28)}$, Portugal ${ }^{(29,30)}$, South Korea ${ }^{(31,32)}$, Sweden ${ }^{(33-37)}$ and USA ${ }^{(17,38,39)}$.

From the 21 studies, a total of 345 patients were included, consisting of 302 healthy control patients and 43 CRS patients (CRSwNP 34 and CRSsNP 9). Eleven studies involved mucus samples; seven collected by nasal lavage fluid washes and four by suction. Ten studies involved sampling of the nasal mucosa, five by mucosal brushing and five by intraoperative biopsies.

A total of 2962 proteins were identified after removal of duplicates in the 21 studies. 549 proteins were identified in mucus samples, consisting of 45 unique to CRS samples, 398 unique to healthy and 106 common in both groups. 2829 proteins were identified in mucosa samples, including six unique to CRS samples, 2771 unique to healthy and 52 common in both groups (Supplement 3).

\section{Critical analysis of included studies}

All 21 studies included in this review focused on the identification and analysis of the nasal mucus and mucosa proteome and all included analysis of control donors and/or CRS patients. Only 
Table 2. Studies investigating proteomics of nasal mucosa.

\begin{tabular}{|c|c|c|c|c|c|c|}
\hline Author & $\begin{array}{l}\text { Disease } \\
\text { Status }\end{array}$ & Patients & Collection Method & MS Method & MS Equipment & $\begin{array}{l}\text { Proteins } \\
\text { Identified }\end{array}$ \\
\hline $\begin{array}{l}\text { Farajzadeh } \\
\text { Deroede } \\
\text { et al. }{ }^{18}\end{array}$ & CRSwNP & 3 & $\begin{array}{l}\text { Biopsy } \\
\text { (Polyp tissue) }\end{array}$ & MALDI-TOF-MS & $\begin{array}{l}\text { Proteome-Analyser } 4700 \text { (Applied Biosystems; } \\
\text { CA, USA) }\end{array}$ & 11 \\
\hline $\begin{array}{l}\text { Gelardi } \\
\text { et al. }{ }^{28}\end{array}$ & Healthy & 4 & $\begin{array}{l}\text { Nasal brushing } \\
\text { (Inferior turbinate) }\end{array}$ & MALDI-TOF-MS & $\begin{array}{l}\text { Voyager DE PRO MS (Applied Biosystems; CA, } \\
\text { USA) }\end{array}$ & 18 \\
\hline $\begin{array}{l}\text { Kim } \\
\text { et al. }{ }^{31}\end{array}$ & CRSwNP & 13 & $\begin{array}{l}\text { Biopsy } \\
\text { (Polyp tissue) }\end{array}$ & Nano-LC-MS/MS & $\begin{array}{l}\text { Agilent } 1100 \text { Series LC/MSD Trap XCT MS } \\
\text { (Agilent Technologies; Ontario, Canada) }\end{array}$ & 15 \\
\hline $\begin{array}{l}\text { Lee } \\
\text { et al. }{ }^{32}\end{array}$ & Healthy & 10 & $\begin{array}{l}\text { Biopsy } \\
\text { (Inferior turbinate) }\end{array}$ & MALDI-TOF MS & $\begin{array}{l}\text { Voyager DE STR MS (Applied biosystems; CA, } \\
\text { USA) }\end{array}$ & 78 \\
\hline $\begin{array}{l}\text { Min-Man } \\
\text { et al. }{ }^{24}\end{array}$ & $\begin{array}{l}\text { Healthy } \\
\text { CRSsNP } \\
\text { CRSwNP }\end{array}$ & $\begin{array}{l}7 \\
7 \\
7\end{array}$ & $\begin{array}{l}\text { Biopsy } \\
\text { (Ethmoid sinuses - CRS, } \\
\text { Middle turbinate - Healthy) }\end{array}$ & $\begin{array}{l}\text { MALDI-TOF MS } \\
\text { ESI-Q-TOF MS }\end{array}$ & N/A & 30 \\
\hline $\begin{array}{l}\text { Ndika } \\
\text { et al. }{ }^{36}\end{array}$ & Healthy & 10 & $\begin{array}{l}\text { Nasal Brushing } \\
\text { (Middle meatus) }\end{array}$ & Nano-LC-MS/MS & $\begin{array}{l}\text { LC (EASY nano LC } 1000 \text { (Proxeon, Thermo Fischer } \\
\text { Scientific; CA, USA) } \\
\text { Electrospray ionization quadrapole-orbitrap MS } \\
\text { (Q Exactive, Thermo Fisher Scientific; CA, USA) }\end{array}$ & $\begin{array}{l}\text { Spring } \\
2090 \\
\text { Autumn } \\
2107\end{array}$ \\
\hline $\begin{array}{l}\text { Roxo-Rosa } \\
\text { et al. }{ }^{29}\end{array}$ & Healthy & 8 & $\begin{array}{l}\text { Nasal Brushing } \\
\text { (Middle meatus) }\end{array}$ & MALDI-TOF MS & $\begin{array}{l}\text { Biflex III (Bruker Daltonik; Bremen, Germany) } \\
\text { Voyager DE STR MS (Applied Biosystems; Ontario, } \\
\text { Canada) }\end{array}$ & 65 \\
\hline $\begin{array}{l}\text { Simoes } \\
\text { et al. }{ }^{30}\end{array}$ & Healthy & 129 & $\begin{array}{l}\text { Nasal Brushing } \\
\text { (Inferior turbinate) }\end{array}$ & Micro-LC-MS/MS & $\begin{array}{l}\text { Thermo LTQ linear ion trap spectrometer } \\
\text { (Thermo Scientific, Waltham, MA) }\end{array}$ & 1482 \\
\hline $\begin{array}{l}\text { Suojalehto } \\
\text { et al. }{ }^{25}\end{array}$ & Healthy & 27 & $\begin{array}{l}\text { Nasal Brushing } \\
\text { (Inferior turbinate) }\end{array}$ & ESI-Q-TOF MS & $\begin{array}{l}\text { LC (EASY nano LC } 1000 \text { (Proxeon, Thermo Fischer } \\
\text { Scientific; CA, USA) } \\
\text { Electrospray ionization quadrapole-orbitrap MS } \\
\text { (Q Exactive, Thermo Fisher Scientific; CA, USA) }\end{array}$ & 77 \\
\hline $\begin{array}{l}\text { Upton DC } \\
\text { et al. }{ }^{17}\end{array}$ & $\begin{array}{l}\text { Healthy } \\
\text { CRSwNP }\end{array}$ & $\begin{array}{l}3 \\
3\end{array}$ & $\begin{array}{l}\text { Biopsy } \\
\text { (Ethmoid, Sphenoid } \\
\text { sinuses) }\end{array}$ & MALDI-TOF MS & $\begin{array}{l}\text { Voyager DE Pro mass spectrometer } \\
\text { (Protein Core Facility) }\end{array}$ & 15 \\
\hline
\end{tabular}

HPLC: High performance liquid chromatography; LC: Liquid Chromatography; MS: Mass spectrometry; LC-MS/MS: Liquid chromatography-tandem mass spectrometry; ESI-Q-TOF MS: Electrospray-ionisation quadrupole time-of-flight mass spectrometry; LC-Q-TOF MS: Liquid chromatography/ quadrupole time-of-flight mass spectrometry; MALDI-TOF MS: Matrix Assisted Laser Desorption/ lonisation mass spectrometry; Spring: Number of proteins identified in spring; Autumn: Number of proteins identified in autumn.

data from healthy and CRS patients was extracted and included in this review. The focus of the included studies was to identify and ascertain differences in the nasal mucus or tissue proteome between CRS patients and controls, however, there was considerable variability in patient demographics and clinical status. Four studies obtained samples from healthy and allergic rhinitis patients before and after pollen seasons to compare changes in proteome $(23,25,33,36)$. The allergic rhinitis protein content was excluded from this review, and only the healthy proteome was included in the overall protein list. One study did not document the number of patients included ${ }^{(27)}$, and two studies did not include patient age ${ }^{(33,35)}$. These three studies consisted of only healthy patients, and the proteins identified were included in the catalogue of proteins in this review (Supplement 2). CRS was diagnosed via nasoendoscopic examination in all cases, with the addition of CT imaging in three studies ${ }^{(17,24,31)}$. Minimal details were given across the included studies regarding polyp grades and CT scores with one study grading polyp severity in CRS patients ${ }^{(18)}$. Studies defined healthy patients who demonstrated no clinical or CT evidence of allergic rhinitis or CRS. One study included two smokers ${ }^{(31)}$ and two studies utilized oral steroids ${ }^{(17,18)}$. These details are summarised in Supplement 4.

There was heterogeneity between studies regarding sample collection and preparation prior to analysis with the mass spectrometer. Nasal mucus was collected via nasal lavage fluid (NLF) in seven studies and suction in four studies (Table 1). Nasal tissue samples were collected as nasal brushings in five studies and biopsy of nasal mucosa (inferior turbinate, polyp, ethmoid or sphenoid sinuses) in five studies (Table 2). Heterogeneity in sample collection method and site of tissue extraction is a potential for confounding bias. Seven studies performed additional immunochemistry on cells to identify cellular changes in healthy and CRS patients $(17,24,28,29,31,32,34)$. These studies identified a range 


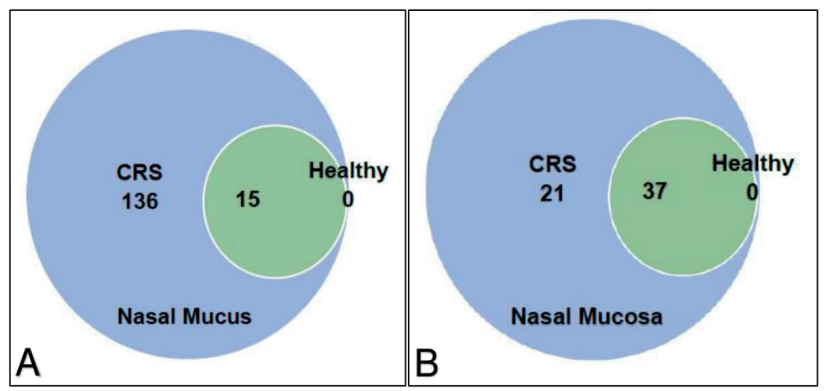

Figure 1. Venn diagram demonstrating total proteins identified in CRS and healthy patients in nasal mucus (A) and nasal mucosa (B).

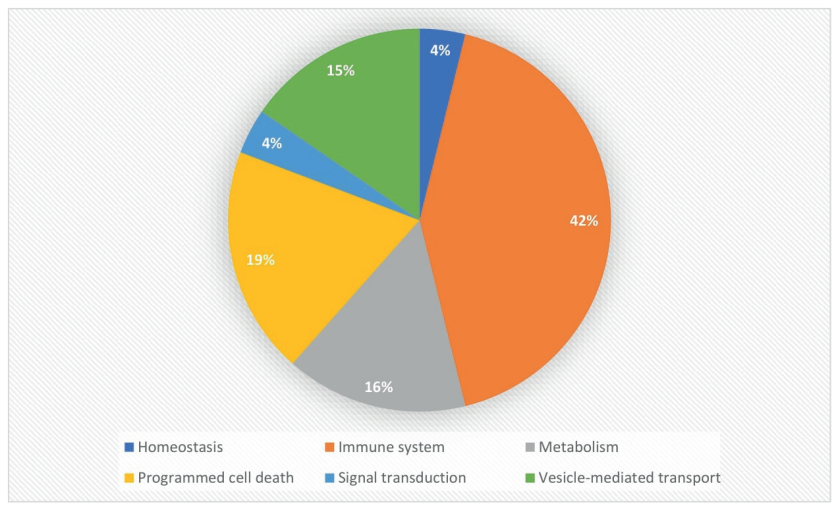

Figure 2. CRS cellular pathways. Twenty-six cellular pathways identified, classified into six common groups. (\%) Percentage of all identified cellular pathways in CRS samples.

of nasal mucosal and inflammatory cells, however, there is limited reporting of which specific cell types were tested during the proteomic experiments.

Nasal tissue sample preparation prior to mass spectrometry analysis was variable, however, followed the same principles across all the studies. Nasal mucus samples were run through $2 \mathrm{D}$ Gel electrophoresis in seven studies ${ }^{(22,23,26,27,33,34,37)}$ Protein spots of interest were cut and underwent trypsin digestion prior to mass spectrometry analysis. Nasal tissue samples all underwent tissue lysis through combinations of lysis buffers and mechanical disruption via sonification. Protein was collected from these lysed cells and run through 2D gel electrophoresis in eight of the studies $(17,18,24,25,28,29,31,32)$.

Various techniques of mass spectrometry were employed across the 21 included studies, where some studies used two different techniques. Ion trap LC-MS/MS was used in ten studies, matrix assisted laser desorption/ ionisation mass spectrometry (MALDI-TOF) was used in ten studies, electrospray-ionisation quadrupole time-of-flight mass spectrometry (ESI-Q-TOF) was used in three studies and liquid chromatography/ quadrupole time-of-flight mass spectrometry (LC-Q-TOF) was used in one

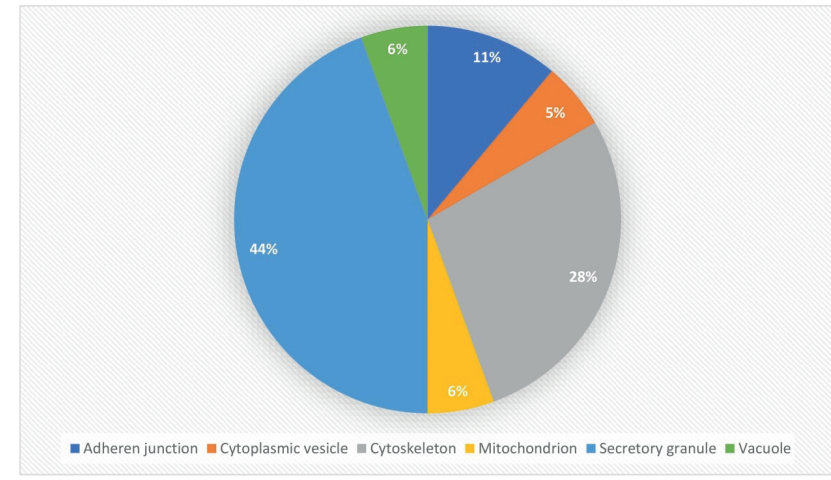

Figure 3. CRS cellular components. Eighteen cellular components classified in six common groups. (\%) Percentage of all identified cellular components in CRS samples.

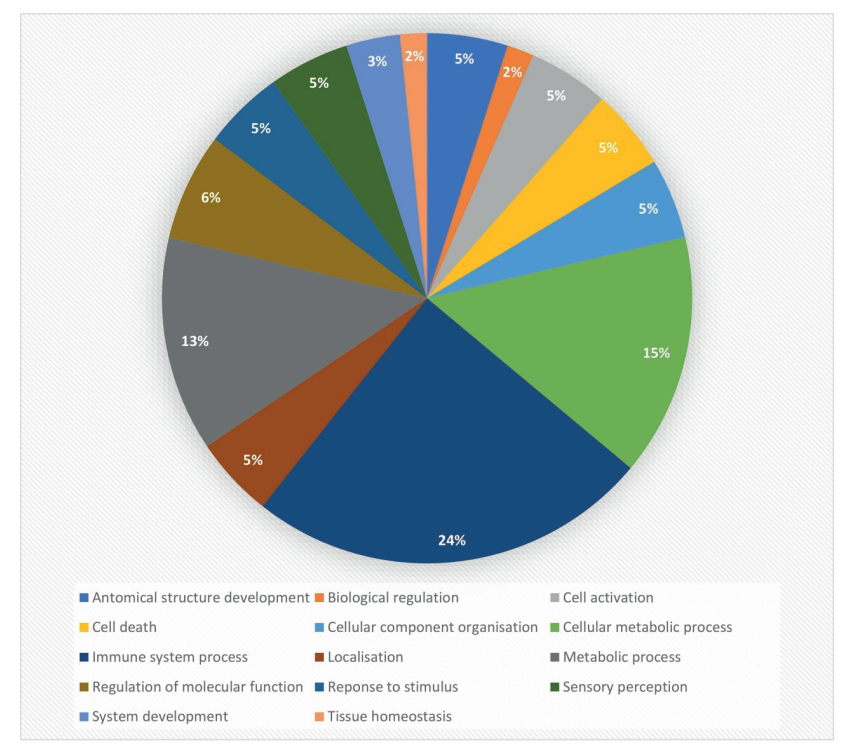

Figure 4. CRS biological processes. Sixty-one biological processes classified into 14 common groups. (\%) Percentage of all identified biological processes in CRS samples.

study (Table 1 and Table 2). Notably, the most recent of these studies was published 8 years ago, and since then, there have been considerable advances in MS equipment speed, resolution and sensitivity. Furthermore, various protein identification criteria were employed, including 2-4 peptides present, 10-20\% sequence coverage and false discovery rates of less than $5 \%$.

\section{Nasal mucus and mucosa proteome gene set enrichment analysis}

There was substantial skew of studies investigating healthy mucus and mucosa samples compared to CRS samples. Thus, GSEA was only performed on the proteins identified in the six studies that sampled CRS patients. Two of these studies investigated nasal mucus ${ }^{(16,38)}$ and four studies examined the nasal mucosa ${ }^{(17,18,}$ 24,31). Three of these six studies directly compared the proteomes of CRS and healthy patients $(16,17,24)$. This was to reduce methodo- 


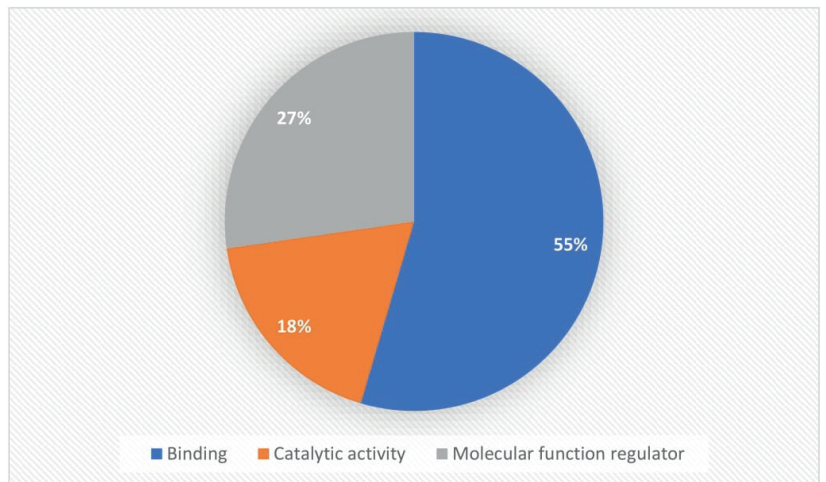

Figure 5. CRS molecular functions. Eleven molecular functions classified into three common groups. (\%) Percentage of all identified molecular functions in CRS samples.

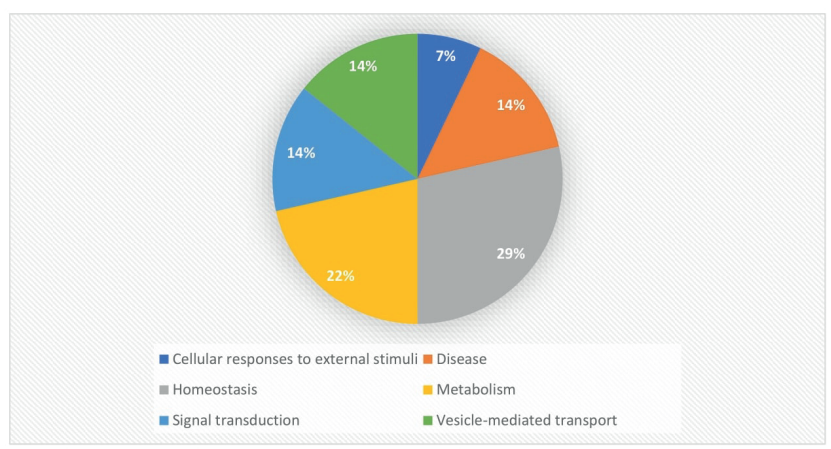

Figure 6. Healthy and CRS cellular pathways. Thirteen cellular pathways identified and stratified into six common pathways. (\%) Percentage of all identified cellular pathways in both healthy and CRS samples.

logical bias in the analysis.

151 proteins were identified in the two studies investigating the nasal mucus proteome (Supplement 5 and Figure 1) (16,38) $^{(136}$ proteins were uniquely expressed in CRS mucus and 15 were found in both CRS and healthy mucus samples whilst no proteins were found to be unique to the healthy mucus proteome. 58 proteins were identified across four studies investigating the proteomics of nasal mucosa ${ }^{(17,18,24,31)} .21$ proteins were uniquely expressed in CRS mucosa, 37 proteins were present in both healthy and CRS mucosa and no proteins were unique to the healthy mucosa proteome (Figure 1).

\section{CRS proteome}

Twenty-six cellular pathways were identified in the 136 uniquely expressed CRS mucus proteins and classified into six common groups (Supplement 5). The pathways included immune system (42\%), programmed cell death (19\%), metabolism (16\%), vesicle mediated transport (15\%), homeostasis (4\%) and signal transduction (4\%) (Figure 2). No significant cellular pathways were identified in CRS mucosa samples.

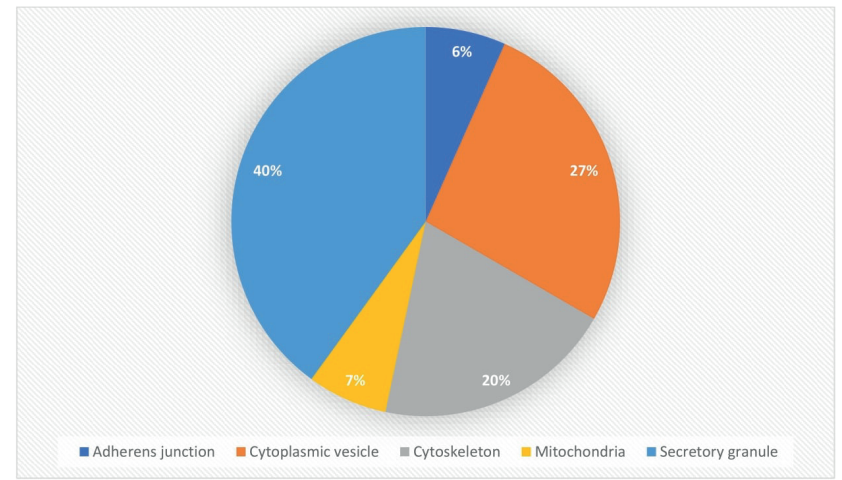

Figure 7. Healthy and CRS cellular components. Fifteen cellular components identified and classified in five common groups. (\%) Percentage of all identified cellular components in both healthy and CRS samples.

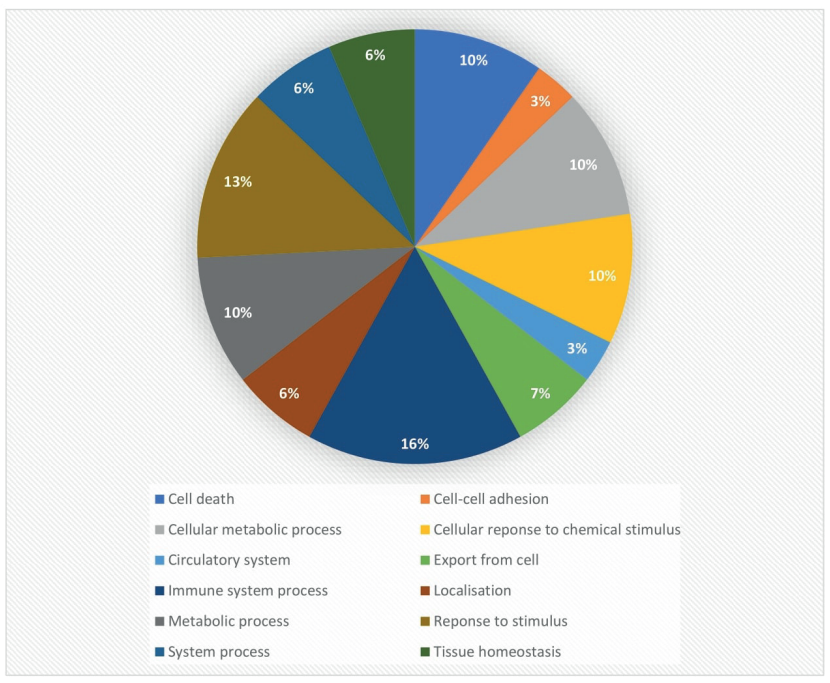

Figure 8. Healthy and CRS biological processes. Thirty-one biological processes classified in twelve common biological processes. (\%) Percentage of all identified biological processes.

Eighteen cellular components were uniquely identified in CRS mucus and mucosa samples, classified into six common groups consisting of secretory granules (44\%), cytoskeleton (28\%), adherens junction (11\%), vacuoles (6\%), mitochondrion (6\%) and cytoplasmic vesicles (5\%) (Supplement 5 and Figure 3).

Sixty-one biological processes were identified in CRS mucus and mucosa samples, stratified into 14 common groups (Supplement 5). These processes included mainly immune system process $(24 \%)$, cellular metabolic process (15\%), metabolic process (13\%), regulation of molecular function (6\%) as well as anatomical structure development, biological regulation, cell activation, cellular component organisation, localisation, function, response to stimulus, sensory perception, system development and tissue homeostasis (all $\leq 5 \%$ ) (Figure 4). 
Eleven molecular functions were significantly present in CRS mucus, classified into three common functions consisting of binding (55\%), molecular function regulation (27\%) and catalytic activity (18\%) (Supplement 5 and Figure 5). No molecular functions were identified in CRS mucosa samples.

\section{Common proteome in CRS and healthy}

All proteins identified in healthy mucus ${ }^{(15)}$ and mucosa ${ }^{(37)}$ samples were also present in CRS mucus and mucosa samples, respectively.

Thirteen cellular pathways were identified in both healthy and CRS mucus and mucosa samples were classifed into six common pathways: homeostasis (29\%), metabolism (22\%), vesicle-mediated transport (14\%), signal transduction (14\%), disease (14\%) and cellular responses to external stimuli (7\%) (Supplement 5 and Figure 6).

Fifteen cellular components were found to be present in both healthy and CRS mucus and mucosa samples. These were grouped into five common groups consisting of secretory granules $(40 \%)$, cytoplasmic vesicles $(27 \%)$, cytoskeleton $(20 \%)$, mitochondrion (7\%) and adherens junctions (6\%) (Supplement 5 and Figure 7).

Thirty-one biological processes were present in both healthy and CRS mucus and mucosa samples (Supplement 5). These were grouped into twelve common biological processes consisting mainly of immune processes (16\%), response to stimulus (13\%), metabolic process (10\%), cellular metabolic process (10\%), cellular response to chemical stimulus (10\%), cell death (10\%), cellular metabolic process (10\%), export from cell (7\%), as well as localisation, system processes, tissue homeostasis and tissue homeostasis, cell-cell adhesion and circulatory system (all $\leq 6 \%$ ) (Figure 8).

Signal recognition particle binding (GO:0005047) was the only significant molecular function present in both CRS and healthy mucus samples (Supplement 5). No other molecular functions were identified in either the healthy or CRS mucosa samples.

\section{Discussion}

Chronic rhinosinusitis is a multifactorial disease believed to be secondary to a complex interplay between pathogens, the immune system and environmental factors ${ }^{(40)}$. This results in chronic mucosal inflammation and recurrent infections. The currently used phenotypic classification which is based on the presence or absence of polyps has been shown to be inadequate in defining the numerous inflammatory subtypes within CRS. To better define the heterogeneous disease process in CRS, there have been numerous studies investigating the pathophysiology and classification of CRS endotypes ${ }^{(7,40)}$. This scoping review has identified 21 studies investigating the nasal mucus and mucosa proteome of healthy and CRS patients that may give insights into the protein composition and down-stream alterations that occur in sinus disease. The various methodologies of all included studies were summarised and critically appraised. There was significant heterogeneity in methodology and patients across the current available literature. Gene set enrichment analysis (GSEA) was conducted on six proteomic studies that had data comparing the proteome of CRS and healthy cohorts ${ }^{(16-18,24,31,38)}$. These results suggest an increased proportion of both innate and adaptive immunologic pathways including complement, phagocytosis and B cell functions, with increases to tissue remodelling pathways, apoptosis and metabolic processes in CRS patients compared to the common proteome group. These differences as well as their associated imbalances in cellular pathways may account for the disease manifestation observed in CRS, however, further studies are required to confirm these findings.

Dysregulation of the innate and adaptive immune system response has been proposed as a contributing factor to the underlying chronic inflammatory state observed in CRS ${ }^{(41-43)}$. Using GSEA this review demonstrated an increased presence of complement activation processes (GO:0006958) and phagocyte activity (GO:0006909) in the CRS group. Both these factors are part of the innate immune system which is vital against inhaled pathogens in the airway. Studies have identified upregulation of complement components and deposition along the basement membrane in CRS nasal polyp tissue ${ }^{(44,45)}$. This deposition is associated with epithelial-to-mesenchymal transition, a form of tissue remodelling observed in CRS mucosa ${ }^{(46,47)}$. Additionally, C5a has been found to induce production of Oncostatin $\mathrm{M}$, a potent cytokine, known to disrupt epithelial barrier integrity in CRSwNP ${ }^{(48)}$. Macrophages and neutrophils are prominent phagocytic cells in CRS, with their dysregulation potentially accounting for the epithelial injury and increased paracellular permeability observed in CRS ${ }^{(41,49,50)}$. The increased expression of these innate immune system pathways in CRS patients may account for the barrier dysfunction associated with the sinonasal inflammatory response. In turn, differences in pathway expression can used to endotype CRS and aid in prognostication and treatment.

Secretory granules are important constituents in phagocyte function in the innate immune response, with similar compositions found between the CRS and common proteome groups following GSEA. Elevated levels of neutrophils observed in CRS patients may lead to over production of neutrophil granules and serine proteases, which may lead to barrier disruption as collateral damage ${ }^{(50)}$. This review identified differences in endopeptidase (GO:0052548) and peptidase (GO:0052547) activity in CRS mucus which was not present in the common group. Neutrophil 
elastase, an endopeptidase, is vital in the fight against pathogens has been associated with epithelial barrier disruption ${ }^{(50)}$. The imbalance between proteases and protease inhibitors at the epithelial barrier is hypothesised to be an initiating and perpetuating factor in inflammatory airway disease ${ }^{(51,52)}$. Further targeted studies are required to determine if differences in protein expression of these inflammatory mediators reflect the increased infiltration of inflammatory cells with consequent barrier disruption.

The adaptive immune response is also implicated in the pathogenesis of CRS. Our GSEA confirms this with increased proportion of B cell activation (GO:0050871) and function (GO:0050853) pathways in CRS mucus samples. These findings correlate with previous studies identifying elevated levels of B cells and plasma infiltrates in CRS ${ }^{(53-55)}$. B cells are fundamental in the adaptive immune response, by evolving into plasma cells or memory B cells upon activation. From research by our department, we have also recently demonstrated immune cell infiltrates clustering into germinal centres called tertiary lymphoid organs (TLO). We proposed that the presence of TLO in nasal polyp samples in recalcitrant CRS patients may be secondary to chronic antigen presentation ${ }^{(56)}$. Studies have speculated the role of TLO in the activation of autoreactive $B$ and $T$ cell clones in chronic inflammation and autoimmunity ${ }^{(42,57,58)}$. Further research is required to understand the contribution of the innate and adaptive immune response in CRS endotypes, and its role of tissue remodelling and the development of polyps.

GSEA also demonstrated an impairment in immune response against pathogen invasion including the defence response biological processes against bacterium (GO:0042742) and fungus (GO:0050832), that were only found to be present in the CRS group. These pathways need to be explored to determine if the proteins involved are downregulated or upregulated in CRS patients. Furthermore, three sensory perception processes identified in CRS mucus were involved with bitter taste. The bitter taste receptor gene family, particularly $T 2 R 38$, has been associated with activation of the local innate immune response through increased mucociliary clearance and bactericidal activity ${ }^{(59,60)}$. It has been proposed that polymorphisms in bitter taste receptors play a role in sinonasal immunity in CRS ${ }^{(61)}$. The impairment in immunological pathways present may account for the impaired innate and adaptive immune response with secondary bacterial dysbiosis observed in CRS ${ }^{(62,63)}$.

Tissue remodelling with associated barrier dysfunction secondary to inflammation is a hallmark feature of CRS ${ }^{(64)}$. Epithelialto-mesenchymal transition (EMT) is a remodelling process where epithelial cells lose their normal morphology, polarity and junctional attachments to neighbouring cells becoming spindle shaped mesenchymal cells in response to chronic inflammation ${ }^{(65)}$. These cellular changes with loss of tight and adherens junctions from CRS mucosa are well documented ${ }^{(66,67)}$. This review identified increased proportion of cytoskeletal and adherens junction components in the CRS group compared to the common proteome group. Furthermore, proteins involved in cell-cell binding played the largest role in molecular functions of the CRS group. These differences may reflect the cellular morphological changes observed in CRS described in the barrier disruption hypothesis of CRS pathogenesis. Lastly, the increased proportion of actin-filaments (GO:0005884) and intermediate filament cytoskeleton (GO:0045111) present in the CRS group may account for increased activity during tissue remodelling. Further research is required to identify differences in expression of barrier and cytoskeletal structure proteins that reflect the changes observed in CRS.

Interestingly, our review also showed an increased expression of programmed cell death pathways and biological processes to be present in CRS patients. Apoptosis has a fundamental role in regulating tissue homeostasis via the elimination of unwanted cells. The apoptotic cleavage pathway of cell adhesion proteins leads to rearrangements of tight junctions, adherens junctions and desmosomes. Immune-mediated apoptosis of intestinal epithelial cells demonstrated increased permeability with associated restructuring of epithelial cells ${ }^{(68)}$. Caspases released during apoptosis causes cleavage of desmosomal proteins leading to disruption of cell-cell contacts. This is followed by remodelling of the intermediate filament cytoskeleton, as a homeostatic mechanism observed in colonic epithelial cells to maintain barrier function ${ }^{(69)}$. These findings correlate with cytokine-mediated changes in tight junctions with increased paracellular permeability present in inflammatory bowel disease (70-72). This pathway may account for the increased paracellular permeability without epithelial cell cytotoxicity observed in human nasal epithelial cells exposed to CRS mucus ${ }^{(6)}$. Further research is required to determine differences in the upstream regulation of apoptotic pathways present in CRS, followed by clarifying if apoptosis-induced morphological changes in nasal epithelial is associated with barrier dysfunction and paracellular permeability.

The dysregulation of the coagulation cascade has been hypothesised to play a role in the pathogenesis of many inflammatory conditions including asthma, rheumatoid arthritis and Crohn's disease ${ }^{(73)}$. This review identified the platelet degranulation pathways with similar distributions in both groups. Elevated expression of platelet-derived growth factor (PDGF) has been identified in nasal polyps suggesting involvement in epithelial proliferation observed in CRS ${ }^{(74)}$. PDGF is chemotactic and mitogenic for fibroblasts, and involved in the pathogenesis of 
fibrosis ${ }^{(75)}$. Additionally, factor XIII-A in the coagulation cascade, present in platelets and macrophages, is also elevated in nasal polyp tissues of CRSwNP patients ${ }^{(76)}$. It was hypothesised that overproduction of factor XIII-A may lead to an accelerated coagulation cascade with subsequent excessive fibrin deposition, leading to tissue remodelling and oedema of the submucosa within nasal polyp tissue ${ }^{(73)}$. Further research is required to ascertain the differences in protein regulation upstream in platelet pathways which may be responsible for the increased fibrin deposition in tissue remodelling.

The maintenance of cellular metabolism is vital in healthy tissue homeostasis. This review identified increased expression of metabolic processes, particularly with carbohydrate metabolism, was present in the CRS-only group. Persistent exposure of nasal epithelial cells to external pathogens and irritants leads to injury with chronic healing and remodelling. Reactive oxygen species released in response to pathogens further add to the oxidative stress in chronic disease ${ }^{(77)}$. This process is counterbalanced by a range of antioxidant pathways identified in both healthy and CRS mucus. Previous studies have demonstrated reduced levels of antioxidant enzymes in CRS patients compared to healthy individuals ${ }^{(78,79)}$. Consequently, the increased energy demand from their epithelial cells require a constant glucose reservoir for the maintenance of cell function and tissue integrity ${ }^{(30)}$. Elevated breath glucose has been identified in patients with cystic fibrosis without diabetes with inflammatory lung changes. It was hypothesised the elevated glucose in airway fluid was due to increased paracellular permeability and glucose leakage into respiratory fluid or impaired glucose removal ${ }^{\left({ }^{80}\right)}$. Further studies are required to investigate imbalances between oxidant and antioxidant processes likely contributing to the inflammation of the epithelium observed in CRS patients ${ }^{\left({ }^{811}\right.}$. Additionally, further research is required to determine if elevated levels of glucose are present in the nasal secretions of CRS patients, and its association with disease severity and barrier dysfunction.

\section{Limitations}

The proteomic analysis of nasal mucosa and mucus samples is a powerful technique in understanding CRS pathogenesis and its endotypes. Heterogeneity in methods of sample collection and location of tissue collected are potential areas of bias. Studies are required to determine if the proteome collected from nasal brushings are as representative as mucosal biopsy specimens. Furthermore, future research is required to determine differences between the nasal mucosa and mucus proteome samples from the same patients. Cellular lysates obtained from nasal mucosa are more challenging to process due to the magnitude of proteins present compared to mucus. Additionally, the protein composition between healthy and CRS cells are likely similar as all cells require similar organelle to survive. The lack of cellular pathways identified from the GSEA of CRS mucosa is likely due to the decreased number of unique proteins ( 21 vs 136) identified and inputted into the analysis. Therefore secretions, such as mucus, may be more representative of the disease process.

Researchers should aim for a standardised mucus and mucosal collection method to reduce sample variability. Targeted methods of sample collection from specific subsites of the nasal cavity, such as with an absorbable sponge or suctioning, will provide more detailed information. Further studies should determine if samples collected from different subsites contain similar quantities of inflammatory cytokines and cells. Furthermore, differing sample processing methods, in conjunction with differing mass spectrometry methods adds confounding bias. Advances in mass spectrometry resolution and sensitivity will allow for the detection of lower abundance proteins present in mucus and mucosa samples to improve our understanding of CRS. GSEA was unable to be performed on all 21 studies due the skew of studies containing only healthy patients. This factor in conjunction with the heterogeneity in sample preparation and analysis across the studies was a potential for cumulative methodological bias. Therefore, this is a potential explanation for the lack of unique healthy proteins identified. The included studies reporting protein qualification was limited and thus this review was only able to comment on the presence of certain proteins and not the quantity. Due to this lack of standardized reporting of up-regulated and/or down-regulated proteins across the studies, we could not collate the data and express it in a meaningful and semi-quantitative way. This is a potential avenue for future research. Thus, GSEA conducted was only capable of presenting pathways present, and not which pathways were up- or down-regulated. Ultimately, more studies with direct comparisons between healthy and CRS proteomes are required. This includes quantitative studies to determine differences in protein and cellular pathway expressions. Lastly, future studies require standardised reporting and methodology to allow improved comparisons in future reviews. Despite these limitations this review has been able to collate a comprehensive list of the nasal proteome, with a descriptive analysis of different cellular processes between healthy and CRS patients.

\section{Conclusion}

Proteomic analysis of nasal mucus and mucosa is a vital step in better understanding the pathophysiology of CRS and its endotypes. This descriptive scoping review has identified 2962 proteins from healthy and CRS patients in the current literature. Preliminary GSEA from the current limited and heterogenous literature suggests a trend of increased presence of immunological, metabolic, tissue remodelling and apoptotic pathways in CRS. Furthermore, the lack of standardisation in methodology across the current literature has been identified. We hope this 
review provides a reference resource of the current literature to improve standardisation of methodology and direct further research to improve the knowledge of CRS pathogenesis and endotypes.

\section{Acknowledgements}

The project was supported by the Adelaide University Research Training Program Scholarship, Bertha Sudholz Scholarship and Garnett Passe Rodney Williams Memorial Foundation Scholarship.

\section{Authorship contribution}

SSK: Study inception, study selection, data collection and analysis, manuscript writing; $A B$ : study selection, data collection and analysis; MR: study selection, data analysis, manuscript writing; NC: data collection and analysis, manuscript writing; ADC: data analysis, manuscript writing; TKC: study selection, data analysis, manuscript writing; PJW: study inception, manuscript writing; SV: study inception, manuscript writing; AJP: study inception, manuscript writing.

\section{Conflict of interest}

The authors declare no conflicts of interest.

\section{References}

1. van Meer $G$, Simons $K$. The function of tight junctions in maintaining differences in lipid composition between the apical and the basolateral cell surface domains of MDCK cells. EMBO journal. 1986;5(7):1455-64.

2. Cereijido M, Valdes J, Shoshani L, Contreras RG. Role of tight junctions in establishing and maintaining cell polarity. Annu Rev Physiol. 1998;60:161-77.

3. Kojima T, Go M, Takano K, Kurose M, Ohkuni T, Koizumi J, et al. Regulation of tight junctions in upper airway epithelium. Biomed Res Int. 2013;2013:947072.

4. Soyka MB, Wawrzyniak P, Eiwegger T, Holzmann D, Treis A, Wanke K, et al. Defective epithelial barrier in chronic rhinosinusitis: the regulation of tight junctions by IFN-gamma and IL-4. J Allergy Clin Immunol. 2012;130(5):1087-96.e10.

5. Tieu DD, Kern RC, Schleimer RP. Alterations in epithelial barrier function and host defense responses in chronic rhinosinusitis. J Allergy Clin Immunol. 2009;124(1):37-42.

6. Kao SS, Ramezanpour M, Bassiouni A, Finnie J, Wormald PJ, Vreugde S, et al. Barrier disruptive effects of mucus isolated from chronic rhinosinusitis patients. Allergy. 2019.

7. Yip J, Monteiro E, Chan Y. Endotypes of chronic rhinosinusitis. Current opinion in otolaryngology \& head and neck surgery. 2019;27(1):14-9.

8. Ramezanpour M, Moraitis S, Smith JLP, Wormald PJ, Vreugde S. Th17 Cytokines Disrupt the Airway Mucosal Barrier in Chronic Rhinosinusitis. Mediators Inflamm. 2016;2016:9798206-.

9. Kim DK, Jin HR, Eun KM, Mo JH, Cho SH, Oh $\mathrm{S}$, et al. The role of interleukin-33 in chronic rhinosinusitis. Thorax. 2017;72(7):635-45.

10. Song W, Wang C, Zhou J, Pan S, Lin S. IL-33 Expression in Chronic Rhinosinusitis with Nasal Polyps and Its Relationship with Clinical Severity. ORL; journal for oto-rhino-laryngology and its related specialties. 2017;79(6):323-30.

11. Beule AG. Physiology and pathophysiology of respiratory mucosa of the nose and the paranasal sinuses. GMS Current Topics in Otorhinolaryngology, Head and Neck
Surgery. 2010;9:Doc07.

12. Fahy JV, Dickey BF. Airway mucus function and dysfunction. N Engl J Med. 2010;363(23):2233-47.

13. Mahdavinia M, Keshavarzian A, Tobin MC Landay AL, Schleimer RP. A comprehensive review of the nasal microbiome in chronic rhinosinusitis (CRS). Clinical and experimental allergy. 2016;46(1):21-41.

14. Stressmann FA, Rogers GB, Chan SW, Howarth PH, Harries PG, Bruce KD, et al. Characterization of bacterial community diversity in chronic rhinosinusitis infections using novel culture-independent techniques. Am J Rhinol Allergy. 2011;25(4):e133-40.

15. Bucher S, Schmid-Grendelmeier P, Soyka MB. Altered Viscosity of Nasal Secretions in Postnasal Drip. Chest. 2019;156(4):659-66.

16. Tewfik MA, Latterich M, DiFalco MR, Samaha M. Proteomics of nasal mucus in chronic rhinosinusitis. Am J Rhinol. 2007;21(6):680-5.

17. Upton DC, Welham NV, Kuo JS, Walker JW, Pasic TR. Chronic Rhinosinusitis With Nasal Polyps: A Proteomic Analysis. Annals of Otology, Rhinology \& Laryngology. 2011;120(12):780-6.

18. Farajzadeh Deroee $A$, Oweinah J, Naraghi M, Hosemann W, Athari B, Volker U, et al. Regression of polypoid nasal mucosa after systemic corticosteroid therapy: a proteomics study. American journal of rhinology \& allergy. 2009;23(5):480-5.

19. R. Development Core Team. R: A Language and Environment for Statistical Computing [Internet]. Vienna AAhwR-po.

20. Kuleshov MV, Jones $M R$, Rouillard $A D$ Fernandez NF, Duan Q, Wang Z, et al. Enrichr: a comprehensive gene set enrichment analysis web server 2016 update. Nucleic acids research. 2016:44(W1):W90-7.

21. Chen EY, Tan CM, Kou Y, Duan Q, Wang Z, Meirelles GV, et al. Enrichr: interactive and collaborative HTML5 gene list enrichment analysis tool. BMC bioinformatics. 2013;14:128.

22. Tomazic PV, Birner-Gruenberger $R$, Leitner A, Obrist B, Spoerk S, Lang-Loidolt D. Nasal mucus proteomic changes reflect altered immune responses and epithelial perme- ability in patients with allergic rhinitis. Journal of Allergy and Clinical Immunology. 2014;133(3):741-50.

23. Tomazic PV, Birner-Gruenberger R, Leitner A, Spoerk S, Lang-Loidolt D. Seasonal proteome changes of nasal mucus reflect perennial inflammatory response and reduced defence mechanisms and plasticity in allergic rhinitis. Journal of proteomics. 2016;133:153-60.

24. Min-Man W, Hong S, Zhi-Qiang X, XuePing F, Chang-Qi L, Dan L. Differential proteomic analysis of nasal polyps, chronic sinusitis, and normal nasal mucosa tissues. Otolaryngology-Head \& Neck Surgery. 2009;141(3):364-8.

25. Suojalehto $H$, Lindström I, Wolff $H$, Puustinen A. Nasal protein profiles in workrelated asthma caused by different exposures. Allergy: European Journal of Allergy and Clinical Immunology. 2018;73(3):65363.

26. Debat H, Eloit C, Blon F, Sarazin B, Henry C, Huet JC, et al. Identification of human olfactory cleft mucus proteins using proteomic analysis. Journal of proteome research. 2007;6(5):1985-96

27. Schoenebeck B, May C, Guldner C, Respondek G, Mollenhauer B, Hoeglinger $\mathrm{G}$, et al. Improved preparation of nasal lavage fluid (NLF) as a noninvasive sample for proteomic biomarker discovery. Biochimica et biophysica acta. 2015;1854(7):741-5.

28. Gelardi M, Siciliano RA, Papa F, Mazzeo MF, De Nitto E, Quaranta N, et al. Proteomic analysis of human nasal mucosa: different expression profile in rhino-pathologic states. European annals of allergy and clinical immunology. 2014;46(5):164-71.

29. Roxo-Rosa M, da Costa G, Luider TM, Scholte BJ, Coelho AV, Amaral MD, et al. Proteomic analysis of nasal cells from cystic fibrosis patients and non-cystic fibrosis control individuals: search for novel biomarkers of cystic fibrosis lung disease. Proteomics. 2006;6(7):2314-25.

30. Simões T, Charro N, Blonder J, Faria D, Couto FM, Chan KC, et al. Molecular profiling of the human nasal epithelium: A proteomics approach. Journal of proteomics. 
2011;75(1):56-69.

31. Kim T, Lee S, Park J, Park S, Jang A, Lee J, et al. Fatty acid binding protein 1 is related with development of aspirin-exacerbated respiratory disease. Allergy: European Journal of Allergy and Clinical Immunology. 2011;66:466-7.

32. Lee JY, Byun JY, Lee SH. Proteomic analysis of normal human nasal mucosa: Establishment of a two-dimensional electrophoresis reference map. Clinical biochemistry. 2009;42(7-8):692-700.

33. Ghafouri B, Irander K, Lindbom J, Tagesson C, Lindahl M. Comparative proteomics of nasal fluid in seasonal allergic rhinitis. Journal of proteome research. 2006:5(2):330-8

34. Lindahl M, Irander K, Tagesson C, Stahlbom B. Nasal lavage fluid and proteomics as means to identify the effects of the irritating epoxy chemical dimethylbenzylamine. Biomarkers 2004;9(1):56-70.

35. Mortstedt H, Karedal MH, Jonsson BA, Lindh $\mathrm{CH}$. Screening method using selected reaction monitoring for targeted proteomics studies of nasal lavage fluid. Journal of proteome research. 2013;12(1):234-47.

36. Ndika J, Airaksinen L, Suojalehto H, Karisola P, Fyhrquist N, Puustinen A, et al. Epithelial proteome profiling suggests the essential role of interferon-inducible proteins in patients with allergic rhinitis. Journal of Allergy and Clinical Immunology. 2017;140(5):1288-98.

37. 37. Wahlen $K$, Fornander $L$, Olausson $P$ Ydreborg K, Flodin U, Graff $P$, et al. Protein profiles of nasal lavage fluid from individuals with work-related upper airway symptoms associated with moldy and damp buildings. Indoor air. 2016;26(5):743-54.

38. Benson LM, Mason CJ, Friedman O, Kita $H$, Bergen HR, 3rd, Plager DA. Extensive fractionation and identification of proteins within nasal lavage fluids from allergic rhinitis and asthmatic chronic rhinosinusitis patients. J Sep Sci. 2009;32(1):44-56.

39. Casado B, Pannell LK, ladarola P, Baraniuk $\mathrm{JN}$. Identification of human nasal mucous proteins using proteomics. Proteomics. 2005;5(11):2949-59.

40. Fokkens WJ. EPOS2020: a major step forward. Rhinology. 2020;58(1):1

41. Lane AP. The role of innate immunity in the pathogenesis of chronic rhinosinusitis. Current allergy and asthma reports 2009;9(3):205-12.

42. Tan BK, Peters AT, Schleimer RP, Hulse KE. Pathogenic and protective roles of $B$ cells and antibodies in patients with chronic rhinosinusitis. The Journal of allergy and clinical immunology. 2018;141(5):1553-60.

43. Schleimer RP. Immunopathogenesis of Chronic Rhinosinusitis and Nasal Polyposis Annual review of pathology. 2017;12:33157.

44. Van Roey GA, Vanison CC, Wu J, Huang JH, Suh LA, Carter RG, et al. Classical complement pathway activation in the nasal tissue of patients with chronic rhinosinusitis. The
Journal of allergy and clinical immunology. 2017;140(1):89-100.e2.

45. Schlosser RJ, Mulligan RM, Casey SE, Varela JC, Harvey RJ, Atkinson C. Alterations in gene expression of complement components in chronic rhinosinusitis. American journal of rhinology \& allergy. 2010;24(1):215.

46. Tang Z, Lu B, Hatch E, Sacks SH, Sheerin NS. C3a mediates epithelial-to-mesenchymal transition in proteinuric nephropathy. Journal of the American Society of Nephrology: JASN. 2009;20(3):593-603.

47. Shin HW, Cho K, Kim DW, Han DH, Khalmuratova R, Kim SW, et al. Hypoxiainducible factor 1 mediates nasal polypogenesis by inducing epithelial-to-mesenchymal transition. Am J Respir Crit Care Med. 2012;185(9):944-54.

48. Pothoven $\mathrm{KL}$, Norton JE, Hulse KE, Suh $L A$, Carter RG, Rocci E, et al. Oncostatin M promotes mucosal epithelial barrier dysfunction, and its expression is increased in patients with eosinophilic mucosal disease. The Journal of allergy and clinical immunology. 2015;136(3):737-46.e4.

49. Van Drunen CM, Mjösberg JM, Segboer $\mathrm{CL}$, Cornet ME, Fokkens WJ. Role of innate immunity in the pathogenesis of chronic rhinosinusitis: Progress and new avenues. Current allergy and asthma reports. 2012;12(2):120-6.

50. Kao SS, Ramezanpour M, Bassiouni A, Wormald PJ, Psaltis AJ, Vreugde S. The effect of neutrophil serine proteases on human nasal epithelial cell barrier function. International forum of allergy \& rhinology. 2019.

51. Kouzaki H, Matsumoto K, Kikuoka H, Kato T, Tojima I, Shimizu S, et al. Endogenous Protease Inhibitors in Airway Epithelial Cells Contribute to Eosinophilic Chronic Rhinosinusitis. Am J Respir Crit Care Med. 2017:195(6):737-47

52. Pfeffer PE, Corrigan CJ. An Imbalance between Proteases and Endogenous Protease Inhibitors in Eosinophilic Airway Disease. Am J Respir Crit Care Med. 2017;195(6):707-8

53. Stoop $A E$, van der Heijden HA, Biewenga J, van der Baan S. Lymphocytes and nonlymphoid cells in human nasal polyps. The Journal of allergy and clinical immunology. 1991;87(2):470-5.

54. Psaltis AJ, Schlosser RJ, Yawn JR, Henriquez $\mathrm{O}$, Mulligan JK. Characterization of B-cell subpopulations in patients with chronic rhinosinusitis. International forum of allergy \& rhinology. 2013;3(8):621-9.

55. Miljkovic D, Psaltis A, Wormald P-J, Vreugde S. Naive and effector B-cell subtypes are increased in chronic rhinosinusitis with polyps. American journal of rhinology \& allergy. 2018;32(1):3-6.

56. Paramasivan S, Lester S, Lau A, Ou J, Psaltis AJ, Wormald P-J, et al. Tertiary lymphoid organs: A\&\#xa0;novel target in patients with chronic rhinosinusitis. Journal of Allergy and Clinical Immunology. 2018;142(5):1673-6.
57. Carragher DM, Rangel-Moreno J, Randall TD. Ectopic lymphoid tissues and local immunity. Semin Immunol. 2008;20(1):26-42.

58. Aloisi F, Pujol-Borrell R. Lymphoid neogenesis in chronic inflammatory diseases. Nat Rev Immunol. 2006;6(3):205-17.

59. Cohen NA. The genetics of the bitter taste receptor T2R38 in upper airway innate immunity and implications for chronic rhinosinusitis. The Laryngoscope. 2017;127(1):44-51

60. Lee RJ, Cohen NA. Sinonasal solitary chemosensory cells "taste" the upper respiratory environment to regulate innate immunity. American journal of rhinology \& allergy. 2014;28(5):366-73

61. Maina IW, Workman AD, Cohen NA. The role of bitter and sweet taste receptors in upper airway innate immunity: Recent advances and future directions. World Journal of Otorhinolaryngology - Head and Neck Surgery. 2018;4(3):200-8.

62. Vickery TW, Ramakrishnan VR. Bacterial Pathogens and the Microbiome. Otolaryngol Clin North Am. 2017;50(1):2947.

63. Sivasubramaniam R, Douglas R. The microbiome and chronic rhinosinusitis. World journal of otorhinolaryngology - head and neck surgery. 2018;4(3):216-21.

64. Van Bruaene N, Bachert C. Tissue remodeling in chronic rhinosinusitis. Current opinion in allergy and clinical immunology. 2011;11(1):8-11.

65. Zhang N, Van Crombruggen K, Gevaert $\mathrm{E}$, Bachert C. Barrier function of the nasal mucosa in health and type-2 biased airway diseases. Allergy. 2016;71(3):295-307.

66. Hupin C, Gohy S, Bouzin C, Lecocq M, Polette $M$, Pilette $C$. Features of mesenchymal transition in the airway epithelium from chronic rhinosinusitis. Allergy. 2014;69(11):1540-9.

67. Rogers GA, Den Beste K, Parkos CA, Nusrat A, Delgaudio JM, Wise SK. Epithelial tight junction alterations in nasal polyposis. International forum of allergy \& rhinology. 2011;1(1):50-4.

68. Abreu MT, Palladino AA, Arnold ET, Kwon RS, McRoberts JA. Modulation of barrier function during Fas-mediated apoptosis in human intestinal epithelial cells. Gastroenterology. 2000;119(6):1524-36.

69. Weiske J, Schoneberg T, Schroder W Hatzfeld M, Tauber R, Huber O. The fate of desmosomal proteins in apoptotic cells. The Journal of biological chemistry. 2001;276(44):41175-81.

70. Colgan SP, Resnick MB, Parkos CA, DelpArcher C, McGuirk D, Bacarra AE, et al. IL-4 directly modulates function of a model human intestinal epithelium. Journal of immunology. 1994;153(5):2122-9.

71. Schmitz H, Fromm M, Bentzel CJ, Scholz P, Detjen K, Mankertz J, et al. Tumor necrosis factor-alpha (TNFalpha) regulates the epithelial barrier in the human intestinal cell line HT-29/B6. Journal of cell science. 1999;112 ( Pt 1):137-46. 
72. Youakim A, Ahdieh M. Interferon-gamma decreases barrier function in T84 cells by reducing ZO-1 levels and disrupting apica actin. The American journal of physiology. 1999;276(5):G1279-88.

73. Kim DY, Cho SH, Takabayashi T, Schleimer RP. Chronic Rhinosinusitis and the Coagulation System. Allergy Asthma Immunol Res. 2015;7(5):421-30

74. Coste A, Wang Q-P, Roudot-Thoraval F, Chapelin C, Bedbeder P, Poron F, et al. Epithelial Cell Proliferation in Nasal Polyps Could Be Up-Regulated by PlateletDerived Growth Factor. The Laryngoscope. 1996;106(5):578-83

75. Antoniades HN, Bravo MA, Avila RE, Galanopoulos T, Neville-Golden J, Maxwell $M$, et al. Platelet-derived growth factor in idiopathic pulmonary fibrosis. The Journa of clinical investigation. 1990;86(4):1055-64.

76. Muszbek L, Bereczky Z, Bagoly Z, Komaromi I, Katona E. Factor XIII: a coagulation factor with multiple plasmatic and cellular functions. Physiological reviews. 2011;91(3):931-
72.

77. Kirkham P, Rahman I. Oxidative stress in asthma and COPD: antioxidants as a therapeutic strategy. Pharmacology \& therapeutics. 2006;111(2):476-94

78. Westerveld GJ, Dekker I, Voss HP, Bast A Scheeren RA. Antioxidant levels in the nasal mucosa of patients with chronic sinusitis and healthy controls. Archives of otolaryngology--head \& neck surgery. 1997;123(2):201-4.

79. Kassim SK, Elbeigermy M, Nasr GF, Khalil R, Nassar M. The role of interleukin-12, and tissue antioxidants in chronic sinusitis. Clinical biochemistry. 2002;35(5):369-75.

80. Baker EH, Clark N, Brennan AL, Fisher DA Gyi KM, Hodson ME, et al. Hyperglycemia and cystic fibrosis alter respiratory fluid glucose concentrations estimated by breath condensate analysis. Journal of Applied Physiology. 2007;102(5):1969-75

81. Fordham MT, Mulligan JK, Casey SE Mulligan RM, Wang EW, Sansoni ER, et al. Reactive oxygen species in chronic rhinosi- nusitis and secondhand smoke exposure. Otolaryngology--head and neck surgery : official journal of American Academy of Otolaryngology-Head and Neck Surgery. 2013;149(4):633.

Alkis Psaltis, MBBS PhD FRACS

Head of Department Otolaryngology

Head and Neck Surgery

The Queen Elizabeth Hospital

28 Woodville Road

Woodville South

South Australia 5011

Australia

E-mail: alkis.psaltis@adelaide.edu.au

This paper contains supplementary materials online: at www.rhinologyjournal.org 


\section{SUPPLEMENTARY INFORMATION}

\section{Supplement 1: Database search.}

\section{Pubmed 26/03/2019}

Sinusitis[mh] OR Sinus*[tw] OR Sinusitides[tw] OR Chronic rhinosinusitis[tw] OR CRS[tw] OR CRSwNP[tw] OR CRSsNP[tw] OR Nasal polyps[mh] OR Papillom*[tw] Polyps[mh] OR Polyp*[tw] OR Nose[mh] OR Nose[tw] OR Nasal*[tw]

AND

Mucus [mh] OR Mucus [tw] OR Bodily secretions [mh] OR Secret* [tw] OR Fluids and Secretions [mh] OR Fluid* [tw] OR Mucus membrane [mh] OR Mucus membrane [tw] OR Respiratory mucosa [mh] OR Respiratory mucosa [tw] OR Nasal mucosa [mh] OR Nasal mucosa [tw] OR Mucosa* [tw] OR Epitheli*[tw] OR Tissue* [tw]

AND

Proteomics [mh] OR Proteom* [tw] OR Proteogenomic [mh] OR

Proteogenomic [tw] OR Secretome[tw]

\section{Embase 26/03/2019}

Sinusitis/syn OR Sinusitis/exp OR Sinus*:ti,ab OR "Chronic rhinosinusitis":ti,ab OR CRS:ti,ab OR CRSsNP:ti,ab OR "Nose polyp"/syn OR "Nose polyp"/exp OR "Nose polyp*":ti,ab OR "Nasal polyp*":ti,ab OR Papillom*:ti,ab Polyp/syn OR Polyp/exp OR Polyp*:ti,ab OR Nose/syn OR Nose/exp OR Nos*:ti,ab

AND

Mucus/syn OR Mucus/exp OR Mucus:ti,ab OR Mucosa*:ti,ab OR "Nose mucus"/syn OR "Nose mucus"/exp OR "Nose fluid":ti,ab OR "Nasal fluid":ti,ab OR Bodily secretions/syn OR Bodily secretions/exp OR Bodily secretion*:ti,ab OR Secret*:ti,ab OR Bodily fluid/syn OR Bodily fluid/exp OR Fluid ${ }^{*}: t i, a b$ OR Mucosa/syn OR Mucosa/exp OR Nose mucosa/syn OR Nose mucosa/exp OR Paranasal sinus mucosa/syn OR Paranasal sinus mucosa/exp OR Nasal tissue/syn OR Nasal tissue/exp OR Tissue ${ }^{*}$ :ti,ab OR Nose epithelium/syn OR Nose epithelium/exp OR Epitheli*:ti,ab AND

Proteomics/syn OR Proteomics/exp OR Proteom*:ti,ab OR Proteogenomics/syn OR Proteogenomics/exp OR Proteogenomic*ti,ab OR Secretome:ti,ab

\section{CINAHL 26/03/2019}

MH Sinusitis OR TI Sinus* OR AB Sinus* ORTA Chronic rhinosinusitis OR AB Chronic rhinosinusitis OR TI CRS OR AB CRS OR TI CRSsNP OR AB CRSsNP OR MH Nasal polyps OR TI Nasal polyp* OR AB Nasal polyp* OR TI Nose polyp* OR AB Nose polyp* OR TI Papillom* OR AB Papillom* OR MH Polyps OR TI Polyp* OR AB Polyp*

AND

MH Mucus OR TI Muc* OR AB Muc* ORTI Nasal muc* OR AB Nasal muc* OR TI Nasal fluid* OR AB Nasal fluid* OR MH Secretions OR MH Fluids and secretions OR TI Secret* OR AB Secret* OR TI Fluid* OR AB Fluid OR MH Mucus membrane OR MH Nasal mucosa OR MH Paranasal sinuses OR MH Tissue OR TI Tissue* OR AB Tissue* OR MH Epithelium OR TI Epitheli*

\author{
OR AB Epitheli*
}

AND

MH Proteomics OR TI Proteomic* OR AB Proteomic* OR TI

Proteogenomic* OR AB Proteogenomic* OR TI Secretome OR AB Secretome

\section{Cochrane 26/03/2019}

Sinus* OR Chronic rhinosinusitis OR CRS OR CRSsNP OR Nose polyp* OR Nasal polyp* OR Papillom* OR Polyp* AND

Mucus* OR Muc* OR Secret* OR Fluid* OR Epitheli* OR Tissue* AND

Proteom* OR Proteogenomic OR Secretome

Supplement 2. PRISMA Flow Diagram. Illustration of the systematic review process of study inclusion and exclusion.
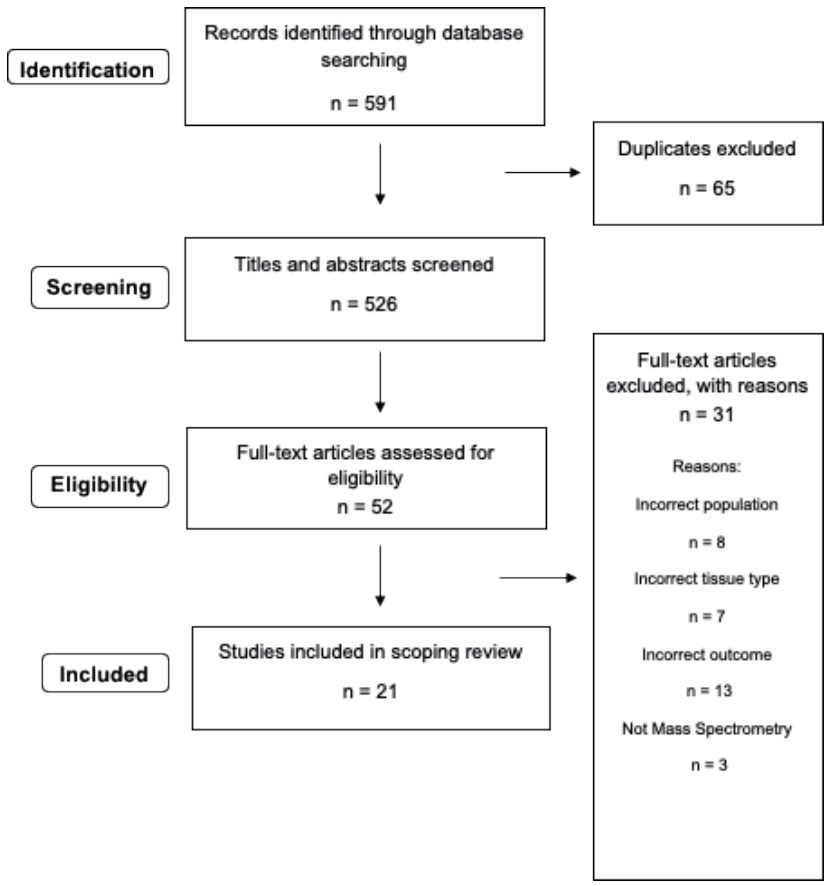


\begin{tabular}{|c|c|c|c|c|c|c|c|}
\hline & Gene & $\begin{array}{l}\text { UniProt Acces- } \\
\text { sion Number }\end{array}$ & Studies & & Gene & $\begin{array}{l}\text { UniProt Acces- } \\
\text { sion Number }\end{array}$ & Studies \\
\hline 1 & $\mathrm{~A} 1 \mathrm{BG}$ & M0R009 & $\mathrm{I}, \mathrm{s}, \mathrm{r}$ & 45 & АСОТ9 & Q9Y305 & I \\
\hline 2 & $\mathrm{~A} 2 \mathrm{M}$ & P01023 & $\mathrm{i}, \mathrm{b}, \mathrm{s}, \mathrm{r}, \mathrm{l}, \mathrm{p}, \mathrm{o}$ & 46 & ACOX3 & 015254 & I \\
\hline 3 & AAAS & Q9NRG9 & o & 47 & ACP1 & P24666 & I \\
\hline 4 & AAMDC & E9PJP1 & I & 48 & ACP2 & P11117 & 0,1 \\
\hline 5 & AARS & P49588 & $\mathrm{s}, \mathrm{l}$ & 49 & ACP6 & Q9NPHO & I \\
\hline 6 & ABCA13 & Q86UQ4 & o & 50 & ACSF2 & Q96CM8 & I \\
\hline 7 & ABCA7 & Q8IZY2 & o & 51 & ACSL1 & P33121 & 0 \\
\hline 8 & ABCB10 & Q9NRK6 & 0 & 52 & ACSL3 & 095573 & 0,1 \\
\hline 9 & ABCB6 & Q9NP58 & o & 53 & ACSL5 & Q9ULC5 & o \\
\hline 10 & $\mathrm{ABCB7}$ & O75027 & o & 54 & ACSS1 & Q9NUB1 & 1 \\
\hline 11 & ABCB8 & Q9NUT2 & o & 55 & ACTA2 & P62736 & 0,1 \\
\hline 12 & $\mathrm{ABCC} 1$ & P33527 & o & 56 & ACTB & P60709 & $n, s, k, c, b, o, t, p, m, a, t, g$ \\
\hline 13 & $\mathrm{ABCC} 3$ & 015438 & o & 57 & ACTBL2 & Q562R1 & $\mathrm{s}$ \\
\hline 14 & $\mathrm{ABCC} 4$ & 015439 & 0 & 58 & ACTC1 & P68032 & $\mathrm{s}$ \\
\hline 15 & $\mathrm{ABCD} 1$ & P33897 & o & 59 & ACTG1 & P63261 & $\mathrm{n}, \mathrm{r}, \mathrm{c}, \mathrm{t}, \mathrm{l}, \mathrm{t}$ \\
\hline 16 & $\mathrm{ABCD} 3$ & P28288 & $\mathrm{I}, \mathrm{O}$ & 60 & ACTN1 & P12814 & $\mathrm{s}, \mathrm{O}, \mathrm{I}$ \\
\hline 17 & $A B C E 1$ & P61221 & I & 61 & ACTN4 & O43707 & $\mathrm{s}, \mathrm{r}, \mathrm{O}, \mathrm{l}, \mathrm{p}$ \\
\hline 18 & ABHD10 & Q9NUJ1 & I & 62 & ACTR1A & P61163 & I \\
\hline 19 & ABHD11 & Q8NFV4 & I & 63 & ACTR1B & P42025 & I \\
\hline 20 & ABHD12 & Q8N2K0 & 0 & 64 & ACTR2 & P61160 & $r, o, l$ \\
\hline 21 & ABHD12B & Q7Z5M8 & $\mathrm{s}$ & 65 & ACTR3 & P61158 & $\mathrm{s}, \mathrm{r}, \mathrm{O}, \mathrm{l}, \mathrm{d}$ \\
\hline 22 & ABHD14B & Q96IU4 & I & 66 & ACY1 & F8WC59 & I \\
\hline 23 & ABHD16A & 095870 & 0 & 67 & ACYP1 & P07311 & I \\
\hline 24 & ABHD6 & Q9BV23 & 0 & 68 & ADAM10 & 014672 & o \\
\hline 25 & $\mathrm{ABI1}$ & Q8IZP0 & I & 69 & ADAR & P55265 & 0,1 \\
\hline 26 & ABRACL & Q9P1F3 & I & 70 & ADCYAP1 & P18509 & j, j \\
\hline 27 & ACAA 1 & P09110 & I & 71 & ADD1 & P35611 & I \\
\hline 28 & ACAD8 & Q9UKU7 & I & 72 & $\mathrm{ADH} 1 \mathrm{~A}$ & P07327 & 0 \\
\hline 29 & ACAD 9 & Q9H845 & 0 & 73 & $\mathrm{ADH} 1 \mathrm{~B}$ & P00325 & I \\
\hline 30 & ACADM & P11310 & $m, o, l$ & 74 & $\mathrm{ADH} 1 \mathrm{C}$ & P00326 & $\mathrm{s}, \mathrm{r}, \mathrm{l}, \mathrm{O}$ \\
\hline 31 & ACADS & P16219 & 0,1 & 75 & ADH5 & P11766 & 1 \\
\hline 32 & ACADSB & P45954 & 1 & 76 & $\mathrm{ADH7}$ & P40394 & $r, s, l$ \\
\hline 33 & ACADVL & P49748 & 0,1 & 77 & ADI1 & Q9BV57 & I \\
\hline 34 & ACAT1 & P24752 & 0,1 & 78 & ADIRF & Q15847 & $\mathrm{I}, \mathrm{O}$ \\
\hline 35 & ACAT2 & Q9BWD1 & I & 79 & ADK & P55263 & I \\
\hline 36 & ACBD3 & Q9H3P7 & 0,1 & 80 & ADPRHL2 & Q9NX46 & 1 \\
\hline 37 & ACBD7 & Q8N6N7 & I & 81 & ADRM1 & Q16186 & 1 \\
\hline 38 & ACIN1 & Q9UKV3 & 0,1 & 82 & ADSL & P30566 & 1 \\
\hline 39 & ACLY & P53396 & 1 & 83 & ADSS & P30520 & I \\
\hline 40 & ACO1 & P21399 & 0,1 & 84 & ADSSL1 & Q8N142 & 1 \\
\hline 41 & $\mathrm{ACO} 2$ & Q99798 & 0,1 & 85 & AFDN & P55196 & I \\
\hline 42 & АСОT13 & Q9NPJ3 & I & 86 & AFG3L2 & Q9Y4W6 & 0 \\
\hline 43 & ACOT2 & P49753 & I & 87 & AFM & P43652 & 1 \\
\hline 44 & ACOT7 & 000154 & 1 & 88 & AGK & Q53H12 & 1,0 \\
\hline
\end{tabular}




\begin{tabular}{|c|c|c|c|}
\hline & Gene & $\begin{array}{l}\text { UniProt Acces- } \\
\text { sion Number }\end{array}$ & Studies \\
\hline 89 & AGL & P35573 & I \\
\hline 90 & AGPAT3 & Q9NRZ7 & o \\
\hline 91 & AGPAT5 & Q9NUQ2 & o \\
\hline 92 & AGPS & 000116 & I \\
\hline 93 & AGR2 & 095994 & $\mathrm{~s}, \mathrm{r}, \mathrm{O}, \mathrm{l}$ \\
\hline 94 & AGR3 & Q8TD06 & $\mathrm{I}, \mathrm{O}$ \\
\hline 95 & AGT & P01019 & $\mathrm{s}, \mathrm{l}$ \\
\hline 96 & $\mathrm{AHCY}$ & P23526 & 1,0 \\
\hline 97 & AHNAK & Q09666 & 0,1 \\
\hline 98 & AHSA1 & 095433 & $s, 1$ \\
\hline 99 & AHSG & P02765 & $\mathrm{s}, \mathrm{r}, \mathrm{u}, \mathrm{l}, \mathrm{a}$ \\
\hline 100 & AIF1 & P55008 & I \\
\hline 101 & AIFM1 & 095831 & 1,0 \\
\hline 102 & AIFM2 & Q9BRQ8 & $b$ \\
\hline 103 & AIMP1 & Q12904 & I \\
\hline 104 & AIMP2 & Q13155 & I \\
\hline 105 & AIP & 000170 & I \\
\hline 106 & AK1 & P00568 & $\mathrm{s}, \mathrm{O}, \mathrm{I}$ \\
\hline 107 & AK2 & P54819 & 0,1 \\
\hline 108 & AK3 & Q9UIJ7 & 0,1 \\
\hline 109 & AK7 & Q96M32 & I \\
\hline 110 & AK8 & Q96MA6 & I \\
\hline 111 & AKAP4 & Q5JQC9 & $\mathrm{S}, \mathrm{O}$ \\
\hline 112 & AKR1A1 & P14550 & $\mathrm{s}, \mathrm{r}, \mathrm{c}, \mathrm{m}, \mathrm{p}, \mathrm{l}, \mathrm{o}$ \\
\hline 113 & AKR1B10 & O60218 & 0,1 \\
\hline 114 & AKR1C1 & Q04828 & $r, s, o, l$ \\
\hline 115 & AKR1C2 & P52895 & $\mathrm{s}, \mathrm{l}, \mathrm{o}, \mathrm{m}$ \\
\hline 116 & AKR1C3 & P42330 & I \\
\hline 117 & AKR7A2 & O43488 & 1,0 \\
\hline 118 & AKT1S1 & Q96B36 & I \\
\hline 119 & ALAD & P13716 & I \\
\hline 120 & ALB & H0YA55 & $\begin{array}{l}u, u, s, r, k, b, c, i, f, u, q, p, m, o \\
, e, q, a\end{array}$ \\
\hline 121 & ALCAM & Q13740 & 0,1 \\
\hline 122 & ALDH16A1 & Q8IZ83 & I \\
\hline 123 & ALDH18A1 & P54886 & 1,0 \\
\hline 124 & ALDH1A1 & P00352 & $c, s, r, o, m, t, e, l, p, t, d$ \\
\hline 125 & ALDH1A3 & P47895 & I \\
\hline 126 & ALDH1B1 & P30837 & I \\
\hline 127 & ALDH1L1 & 075891 & I \\
\hline 128 & ALDH2 & P05091 & $b, o, l, e$ \\
\hline 129 & ALDH3A 1 & P30838 & $r, s, c, p, e, o, l$ \\
\hline 130 & ALDH3A2 & P51648 & 0,1 \\
\hline 131 & ALDH3B1 & P43353 & 0,1 \\
\hline 132 & ALDH4A1 & P30038 & $\mathrm{g}$ \\
\hline 133 & ALDH5A 1 & P51649 & 0,1 \\
\hline 134 & ALDH7A1 & P49419 & 0,1 \\
\hline
\end{tabular}

\begin{tabular}{|c|c|c|c|}
\hline & Gene & $\begin{array}{l}\text { UniProt Acces- } \\
\text { sion Number }\end{array}$ & Studies \\
\hline 135 & ALDH8A 1 & Q9H2A2 & $\mathrm{m}$ \\
\hline 136 & ALDH9A 1 & P49189 & 1,0 \\
\hline 137 & ALDOA & P04075 & s,r,o,p,l,a \\
\hline 138 & ALDOC & P09972 & $\mathrm{s}, \mathrm{O}, \mathrm{l}$ \\
\hline 139 & ALG1 & Q9BT22 & 0,1 \\
\hline 140 & ALG14 & Q96F25 & 0 \\
\hline 141 & ALG2 & Q9H553 & 0 \\
\hline 142 & ALG5 & Q9Y673 & 0 \\
\hline 143 & ALG9 & Q9H6U8 & 0 \\
\hline 144 & ALOX15 & P16050 & $\mathrm{s}, \mathrm{r}, \mathrm{O}, \mathrm{I}$ \\
\hline 145 & ALOX5AP & P20292 & 0 \\
\hline 146 & ALPL & P05186 & 0 \\
\hline 147 & ALYREF & Q86V81 & I \\
\hline 148 & AMBN & Q9NP70 & s \\
\hline 149 & AMBP & P02760 & I \\
\hline 150 & AMDHD2 & Q9Y303 & I \\
\hline 151 & AMY1A & P04745 & $k, l$ \\
\hline 152 & AMY1B & P04745 & k,l \\
\hline 153 & AMY1C & P04745 & k,l \\
\hline 154 & AMY2B & P19961 & $\mathrm{a}$ \\
\hline 155 & ANG & P03950 & I \\
\hline 156 & ANK1 & P16157 & o \\
\hline 157 & ANK3 & Q12955 & 0,1 \\
\hline 158 & ANKEF1 & Q9NU02 & s \\
\hline 159 & ANKS1A & Q92625 & I \\
\hline 160 & ANP32A & P39687 & I \\
\hline 161 & ANP32B & Q92688 & I \\
\hline 162 & ANP32E & Q9BTT0 & I \\
\hline 163 & ANPEP & P15144 & 0,1 \\
\hline 164 & ANXA1 & P04083 & $n, s, r, k, c, l, p, m, t, o, a, t$ \\
\hline 165 & ANXA11 & P50995 & 0,1 \\
\hline 166 & ANXA2 & P07355 & $\mathrm{n}, \mathrm{s}, \mathrm{r}, \mathrm{b}, \mathrm{c}, \mathrm{p}, \mathrm{m}, \mathrm{l}, \mathrm{o}, \mathrm{a}$ \\
\hline 167 & ANXA3 & P12429 & $\mathrm{n}, \mathrm{s}, \mathrm{r}, \mathrm{c}, \mathrm{p}, \mathrm{l}, \mathrm{O}$ \\
\hline 168 & ANXA4 & P09525 & $\mathrm{I}, \mathrm{O}$ \\
\hline 169 & ANXA5 & P08758 & $s, r, p, m, l, o$ \\
\hline 170 & ANXA6 & P08133 & $\mathrm{I}, \mathrm{O}$ \\
\hline 171 & ANXA7 & P20073 & $c, O, I$ \\
\hline 172 & ANXA8 & P13928 & I \\
\hline 173 & AP1B1 & $\mathrm{H} 7 \mathrm{C034}$ & I \\
\hline 174 & AP1G1 & B4DGE1 & $\mathrm{I}, \mathrm{O}$ \\
\hline 175 & AP1M1 & Q9BXS5 & I \\
\hline 176 & $\mathrm{AP} 1 \mathrm{M} 2$ & Q9Y6Q5 & I \\
\hline 177 & $\mathrm{AP} 2 \mathrm{~A} 1$ & 095782 & $\mathrm{I}, \mathrm{O}$ \\
\hline 178 & AP2B1 & P63010 & I \\
\hline 179 & AP2M1 & Q96CW1 & I \\
\hline 180 & $\mathrm{AP} 2 \mathrm{~S} 1$ & P53680 & I \\
\hline 181 & AP3B1 & 000203 & $\mathrm{I}, \mathrm{O}$ \\
\hline
\end{tabular}




\begin{tabular}{|c|c|c|c|c|c|c|c|}
\hline & Gene & $\begin{array}{l}\text { UniProt Acces- } \\
\text { sion Number }\end{array}$ & Studies & & Gene & $\begin{array}{l}\text { UniProt Acces- } \\
\text { sion Number }\end{array}$ & Studies \\
\hline 182 & AP3D1 & 014617 & 1 & 229 & ARRB1 & P49407 & 1 \\
\hline 183 & APCS & P02743 & $p, l$ & 230 & ARSD & P51689 & o \\
\hline 184 & APEH & P13798 & I & 231 & ASAH1 & Q13510 & $\mathrm{s}, \mathrm{O}, \mathrm{l}$ \\
\hline 185 & APEX1 & P27695 & 0,1 & 232 & ASL & P04424 & 1 \\
\hline 186 & API5 & Q9BZZ5 & 1 & 233 & ASMT & P46597 & $\mathrm{I}, \mathrm{I}$ \\
\hline 187 & APMAP & Q9HDC9 & 0,1 & 234 & ASNA1 & O43681 & I \\
\hline 188 & APOA1 & P02647 & $r, f, s, k, k, u, b, c, o, t, l, t$ & 235 & ASPH & Q12797 & o \\
\hline 189 & APOA2 & P02652 & $\mathrm{r}, \mathrm{s}, \mathrm{l}$ & 236 & ASPRV1 & Q53RT3 & a \\
\hline 190 & APOA4 & P06727 & $c, l$ & 237 & ASRGL1 & Q7L266 & 1 \\
\hline 191 & APOB & P04114 & I & 238 & ASS1 & P00966 & $1, p, 0$ \\
\hline 192 & APOC3 & P02656 & $\mathrm{h}$ & 239 & ATAD3A & Q9NVI7 & $\mathrm{l}, \mathrm{O}$ \\
\hline 193 & APOD & P05090 & b & 240 & ATG3 & Q9NT62 & I \\
\hline 194 & $\mathrm{APOH}$ & P02749 & $\mathrm{r}, \mathrm{l}$ & 241 & ATG7 & 095352 & 1 \\
\hline 195 & APOL2 & Q9BQE5 & o & 242 & ATIC & P31939 & 0,1 \\
\hline 196 & APOOL & Q6UXV4 & o & 243 & ATL3 & F5H6I7 & I \\
\hline 197 & APPL2 & Q8NEU8 & I & 244 & ATOX1 & 000244 & I \\
\hline 198 & APRT & P07741 & $p, o, l$ & 245 & ATP12A & P54707 & o \\
\hline 199 & AQP5 & P55064 & o & 246 & ATP13A1 & Q9HD20 & o \\
\hline 200 & ARCN1 & P48444 & 0,1 & 247 & ATP1A1 & P05023 & 0,1 \\
\hline 201 & ARF1 & P84077 & $\mathrm{r}, \mathrm{O}$ & 248 & ATP1B1 & P05026 & 0,1 \\
\hline 202 & ARF3 & P61204 & $s, 1$ & 249 & ATP1B3 & P54709 & 0,1 \\
\hline 203 & ARF4 & P18085 & I & 250 & ATP2A1 & 014983 & o \\
\hline 204 & ARF6 & P62330 & I & 251 & ATP2A2 & P16615 & 0,1 \\
\hline 205 & ARFGAP3 & Q9NP61 & I & 252 & ATP2A3 & Q93084 & 0,1 \\
\hline 206 & ARFGEF1 & Q9Y6D6 & I & 253 & ATP2B1 & P20020 & o \\
\hline 207 & ARFGEF3 & Q5TH69 & o & 254 & ATP2B4 & P23634 & 0,1 \\
\hline 208 & ARFIP1 & P53367 & I & 255 & ATP2C1 & P98194 & o \\
\hline 209 & ARFIP2 & P53365 & I & 256 & ATP5F1A & P25705 & $\mathrm{s}, \mathrm{l}, \mathrm{O}$ \\
\hline 210 & ARHGAP1 & Q07960 & $\mathrm{l}, \mathrm{O}$ & 257 & ATP5F1B & P06576 & $\mathrm{r}, \mathrm{c}, \mathrm{s}, \mathrm{m}, \mathrm{l}, \mathrm{O}$ \\
\hline 211 & ARHGAP18 & Q8N392 & I & 258 & ATP5F1C & P36542 & $\mathrm{I}, \mathrm{O}$ \\
\hline 212 & ARHGDIA & P52565 & $r, s, o, j, l, m, j$ & 259 & ATP5MD & Q96IX5 & 1 \\
\hline 213 & ARHGDIB & P52566 & $n, r, s, o, t, l, j, j, t, j$ & 260 & ATP5ME & P56385 & $\mathrm{l}, \mathrm{O}$ \\
\hline 214 & ARHGEF16 & Q5VV41 & I & 261 & ATP5MF & P56134 & $\mathrm{l}, \mathrm{O}$ \\
\hline 215 & ARID3B & Q8IVW6 & s & 262 & ATP5MG & O75964 & $\mathrm{l}, \mathrm{O}$ \\
\hline 216 & ARL1 & P40616 & I & 263 & ATP5PB & P24539 & $\mathrm{l}, \mathrm{O}$ \\
\hline 217 & ARL3 & P36405 & I & 264 & ATP5PD & 075947 & $m, o, l, j$ \\
\hline 218 & ARL6IP5 & 075915 & 0,1 & 265 & ATP5PF & P18859 & I \\
\hline 219 & ARMCX3 & Q9UH62 & o & 266 & ATP5PO & P48047 & $\mathrm{I}, \mathrm{O}$ \\
\hline 220 & ARMT1 & Q9H993 & I & 267 & ATP6AP1 & Q15904 & o \\
\hline 221 & ARPC1A & Q92747 & I & 268 & ATP6V0A1 & Q93050 & o \\
\hline 222 & ARPC1B & 015143 & 1 & 269 & ATP6V0A2 & Q9Y487 & o \\
\hline 223 & ARPC2 & 015144 & 0,1 & 270 & ATP6V0A4 & Q9HBG4 & o \\
\hline 224 & ARPC3 & 015145 & 0,1 & 271 & ATP6V0C & P27449 & o \\
\hline 225 & ARPC4 & P59998 & $r, o, l$ & 272 & ATP6V0D1 & P61421 & $\mathrm{h}$ \\
\hline 226 & ARPC5 & 015511 & $\mathrm{~s}, \mathrm{l}$ & 273 & ATP6V1A & P38606 & $\mathrm{o}, \mathrm{l,h}$ \\
\hline 227 & ARPC5L & Q9BPX5 & I & 274 & ATP6V1B2 & P21281 & 1 \\
\hline 228 & ARPP19 & P56211 & 1 & 275 & ATP6V1C1 & P21283 & 1 \\
\hline
\end{tabular}




\begin{tabular}{|c|c|c|c|c|c|c|c|}
\hline & Gene & $\begin{array}{l}\text { UniProt Acces- } \\
\text { sion Number }\end{array}$ & Studies & & Gene & $\begin{array}{l}\text { UniProt Acces- } \\
\text { sion Number }\end{array}$ & Studies \\
\hline 276 & ATP6V1D & Q9Y5K8 & I & 323 & BRCC3 & P46736 & I \\
\hline 277 & ATP6V1E1 & P36543 & 0 & 324 & BRI3BP & Q8WY22 & 0 \\
\hline 278 & ATP6V1F & Q16864 & I & 325 & BROX & Q5VW32 & I \\
\hline 279 & ATP6V1H & Q9UI12 & I & 326 & BSG & P35613 & 0 \\
\hline 280 & ATP7B & P35670 & 0 & 327 & BTF3 & P20290 & I \\
\hline 281 & ATP8B1 & 043520 & 0 & 328 & BUB3 & 043684 & I \\
\hline 282 & ATP9A & 075110 & 0 & 329 & BUD31 & P41223 & 1 \\
\hline 283 & AZGP1 & P25311 & b,u,r,k,s,l,p,a & 330 & BZW2 & Q9Y6E2 & I \\
\hline 284 & AZU1 & P20160 & s & 331 & C11orf54 & Q9HOW9 & I \\
\hline 285 & $\mathrm{~B} 2 \mathrm{M}$ & P61769 & $\mathrm{f}, \mathrm{b}, \mathrm{s}, \mathrm{r}, \mathrm{k}, \mathrm{l}, \mathrm{a}$ & 332 & C12orf10 & Q9HB07 & I \\
\hline 286 & B3GAT3 & 094766 & 0 & 333 & C1QB & P02746 & 1 \\
\hline 287 & B3GNT3 & Q9Y2A9 & 0 & 334 & C1QBP & Q07021 & $\mathrm{I}, \mathrm{O}$ \\
\hline 288 & B4GALT4 & 060513 & 0 & 335 & C1QC & P02747 & $\mathrm{I}, \mathrm{p}$ \\
\hline 289 & BAG1 & Q99933 & I & 336 & $\mathrm{C} 1 \mathrm{R}$ & P00736 & 1 \\
\hline 290 & BAG3 & 095817 & I & 337 & C1orf87 & Q8N0U7 & 1 \\
\hline 291 & BAG5 & G3V274 & I & 338 & C21orf59- & H7BZW1 & I \\
\hline 292 & BAG6 & P46379 & I & & TCP10L & & \\
\hline 293 & BAIAP2 & Q9UQB8 & I & 339 & $\mathrm{C} 2 \mathrm{CD} 2 \mathrm{~L}$ & 014523 & 0 \\
\hline 294 & BANF1 & 075531 & I & 340 & $\mathrm{C} 3$ & P01024 & $s, r, k, b, p, l, o$ \\
\hline 295 & BAP18 & Q8IXM2 & I & 341 & C4A & POCOL4 & $b, l, o$ \\
\hline 296 & BASP1 & P80723 & $r, k, o, I$ & 342 & $\mathrm{C} 4 \mathrm{~B}$ & P0C0L5 & $s, r, l$ \\
\hline 297 & BBS1 & Q8NFJ9 & I & 343 & C4BPA & P04003 & $\mathrm{I}, \mathrm{O}$ \\
\hline 298 & BCAM & P50895 & 0 & 344 & C4BPB & P20851 & $\mathrm{h}$ \\
\hline 299 & BCAP29 & Q9UHQ4 & 0 & 345 & C4B_2 & P0C0L5 & $s, r, l$ \\
\hline 300 & BCAP31 & P51572 & $\mathrm{I}, \mathrm{O}$ & 346 & C5 & P01031 & $s, I$ \\
\hline 301 & BCAS1 & 075363 & $\mathrm{I}, \mathrm{O}$ & 347 & C6 & P13671 & 1 \\
\hline 302 & BCAT2 & 015382 & I & 348 & $\mathrm{C7}$ & P10643 & 1 \\
\hline 303 & BCCIP & Q9P287 & I & 349 & $\mathrm{C} 8 \mathrm{~B}$ & P07358 & 1 \\
\hline 304 & $\mathrm{BDH} 1$ & Q02338 & 0,1 & 350 & C8G & P07360 & 1 \\
\hline 305 & $\mathrm{BDH} 2$ & Q9BUT1 & I & 351 & C9 & P02748 & 1 \\
\hline 306 & BFSP1 & Q12934 & $\mathrm{h}$ & 352 & C9orf64 & Q5T6V5 & 1 \\
\hline 307 & BHMT & E5RJH0 & $\mathrm{g}$ & 353 & $\mathrm{CA} 1$ & P00915 & $c, t, l, o, t$ \\
\hline 308 & BID & P55957 & 1 & 354 & CA13 & Q8N1Q1 & I \\
\hline 309 & BLMH & Q13867 & I & 355 & CA2 & P00918 & $b, l, o$ \\
\hline 310 & BLVRA & P53004 & I & 356 & CAB39 & Q9Y376 & 1 \\
\hline 311 & BLVRB & P30043 & 0,1 & 357 & CACYBP & Q9HB71 & I \\
\hline 312 & BOLA2 & Q9H3K6 & I & 358 & CAD & P27708 & $\mathrm{I}, \mathrm{O}$ \\
\hline 313 & BOLA2B & Q9H3K6 & I & 359 & CALM1 & P0DP23 & $\mathrm{s}, \mathrm{r}, \mathrm{l}, \mathrm{a}$ \\
\hline 314 & BPGM & P07738 & s & 360 & CALM2 & P0DP24 & o \\
\hline 315 & $\mathrm{BPHL}$ & Q86WA6 & I & 361 & CALML3 & P27482 & 0,1 \\
\hline 316 & BPI & P17213 & $b, r, s, o$ & 362 & CALML5 & Q9NZT1 & $r, k, l, a$ \\
\hline 317 & BPIFA1 & Q9NP55 & $s, r, b, f, l, p, m, e, o, j, a, j$ & 363 & CALR & P27797 & $c, m, o, I$ \\
\hline 318 & BPIFB1 & Q8TDL5 & $s, k, r, b, l, o$ & 364 & CALU & 043852 & o,l \\
\hline 319 & BPIFB2 & Q8N4F0 & $s, r, k, q, l, q$ & 365 & CAMK2D & Q13557 & I \\
\hline 320 & BPIFB3 & P59826 & $S$ & 366 & CAMP & P49913 & $\mathrm{s}, \mathrm{O}$ \\
\hline 321 & BPIFB4 & P59827 & $s, r, k, l, o$ & 367 & CAND1 & Q86VP6 & $\mathrm{I}, \mathrm{O}$ \\
\hline 322 & BPNT1 & 095861 & $\mathrm{l}, \mathrm{m}$ & 368 & CANX & P27824 & 0,1 \\
\hline
\end{tabular}




\begin{tabular}{|c|c|c|c|c|c|c|c|}
\hline & Gene & $\begin{array}{l}\text { UniProt Acces- } \\
\text { sion Number }\end{array}$ & Studies & & Gene & $\begin{array}{l}\text { UniProt Acces- } \\
\text { sion Number }\end{array}$ & Studies \\
\hline 369 & CAP1 & Q01518 & $\mathrm{r}, \mathrm{s}, \mathrm{O}, \mathrm{l}$ & 416 & CCT5 & P48643 & $c, 1,0$ \\
\hline 370 & CAPG & P40121 & $\mathrm{s}, \mathrm{o}, \mathrm{l}$ & 417 & ССТ6А & P40227 & I \\
\hline 371 & CAPN1 & P07384 & 0,1 & 418 & CCT7 & Q99832 & I \\
\hline 372 & CAPN13 & Q6MZZ7 & I & 419 & ССТ8 & P50990 & o,l,d \\
\hline 373 & CAPN2 & P17655 & 0,1 & 420 & CD14 & P08571 & 0,1 \\
\hline 374 & CAPN5 & 015484 & o & 421 & CD2AP & Q9Y5K6 & I \\
\hline 375 & CAPNS1 & P04632 & $\mathrm{s}, \mathrm{l}, \mathrm{O}$ & 422 & CD38 & P28907 & o \\
\hline 376 & CAPRIN1 & Q14444 & I & 423 & CD44 & P16070 & 0,1 \\
\hline 377 & CAPS & Q13938 & $\mathrm{s}, \mathrm{r}, \mathrm{c}, \mathrm{l}, \mathrm{p}, \mathrm{o}, \mathrm{m}$ & 424 & CD47 & Q08722 & o \\
\hline 378 & CAPZA 1 & P52907 & $\mathrm{s}, \mathrm{O}, \mathrm{I}$ & 425 & CD59 & P13987 & 0,1 \\
\hline 379 & CAPZA2 & P47755 & I & 426 & CD5L & O43866 & I \\
\hline 380 & CAPZB & P47756 & $\mathrm{s}, \mathrm{o}, \mathrm{l}$ & 427 & CD63 & P08962 & o \\
\hline 381 & CARHSP1 & Q9Y2V2 & I & 428 & CD74 & P04233 & 0,1 \\
\hline 382 & CARMIL1 & Q5VZK9 & 1 & 429 & CD81 & P60033 & o \\
\hline 383 & CARS & P49589 & I & 430 & CD82 & P27701 & o \\
\hline 384 & CASK & 014936 & I & 431 & CD9 & P21926 & 0,1 \\
\hline 385 & CASP1 & P29466 & I & 432 & CD97 & P48960 & o \\
\hline 386 & CASP12 & Q6UXS9 & s & 433 & $\mathrm{CDA}$ & P32320 & $\mathrm{s}, \mathrm{r}$ \\
\hline 387 & CASP14 & P31944 & $r, k, a$ & 434 & CDC37 & Q16543 & 0,1 \\
\hline 388 & CASP3 & P42574 & I & 435 & $\mathrm{CDC} 42$ & P60953 & I \\
\hline 389 & CASP7 & P55210 & I & 436 & CDC5L & Q99459 & I \\
\hline 390 & CAST & P20810 & 0,1 & 437 & $\mathrm{CDCP} 1$ & Q9H5V8 & o \\
\hline 391 & CAT & P04040 & $s, r, p, o, l, t, t$ & 438 & $\mathrm{CDH} 1$ & P12830 & 0,1 \\
\hline 392 & CBR1 & P16152 & $\mathrm{s}, \mathrm{r}, \mathrm{o}, \mathrm{l}$ & 439 & CDIPT & 014735 & o \\
\hline 393 & CBR3 & O75828 & I & 440 & CDK5 & Q00535 & I \\
\hline 394 & CBS & P35520 & $\mathrm{h}$ & 441 & CDK5RAP3 & Q96JB5 & I \\
\hline 395 & СBX3 & Q13185 & 0,1 & 442 & CDKL1 & Q00532 & s \\
\hline 396 & CBX5 & P45973 & I & 443 & CDS1 & Q92903 & 0,1 \\
\hline 397 & CC2D1A & Q6P1N0 & 1 & 444 & CDS2 & O95674 & o \\
\hline 398 & CCAR2 & Q8N163 & 0,1 & 445 & CDV3 & Q9UKY7 & I \\
\hline 399 & CCDC102B & Q68D86 & s & 446 & CEACAM5 & P06731 & 0,1 \\
\hline 400 & CCDC124 & Q96CT7 & I & 447 & CENPF & P49454 & $\mathrm{m}$ \\
\hline 401 & CCDC151 & A5D8V7 & I & 448 & CEP128 & Q6ZU80 & o \\
\hline 402 & CCDC17 & Q96LX7 & I & 449 & CEP131 & Q9UPN4 & o \\
\hline 403 & CCDC170 & Q8IYT3 & o & 450 & CEP135 & Q66GS9 & $\mathrm{l}, \mathrm{O}$ \\
\hline 404 & CCDC171 & Q6TFL3 & s & 451 & CERS2 & Q96G23 & o \\
\hline 405 & $\mathrm{CCDC} 22$ & 060826 & I & 452 & CES1 & P23141 & $\mathrm{s}, \mathrm{O}, \mathrm{l}$ \\
\hline 406 & CCDC39 & Q9UFE4 & $\mathrm{l}, \mathrm{I}$ & 453 & CES2 & 000748 & 0,1 \\
\hline 407 & CCDC47 & Q96A33 & o & 454 & CETN2 & P41208 & 1 \\
\hline 408 & CCDC6 & Q16204 & I & 455 & CFAP20 & Q9Y6A4 & I \\
\hline 409 & CCDC78 & A2IDD5 & I & 456 & CFAP52 & Q8N1V2 & I \\
\hline 410 & CCDC81 & Q6ZN84 & $\mathrm{m}$ & 457 & CFAP57 & Q96MR6 & I \\
\hline 411 & CCND1 & P24385 & $\mathrm{h}$ & 458 & CFAP70 & Q5T0N1 & I \\
\hline 412 & CCS & 014618 & I & 459 & CFB & P00751 & $r, u, s, p, l$ \\
\hline 413 & ССТ2 & P78371 & $c, 0, I$ & 460 & $\mathrm{CFH}$ & Р08603 & I \\
\hline 414 & ССТ3 & P49368 & 0,1 & 461 & CFI & P05156 & 1 \\
\hline 415 & СCT4 & P50991 & 0,1 & 462 & CFL1 & P23528 & $\mathrm{s}, \mathrm{r}, \mathrm{o}, \mathrm{l}, \mathrm{p}$ \\
\hline
\end{tabular}




\begin{tabular}{|c|c|c|c|c|c|c|c|}
\hline & Gene & $\begin{array}{l}\text { UniProt Acces- } \\
\text { sion Number }\end{array}$ & Studies & & Gene & $\begin{array}{l}\text { UniProt Acces- } \\
\text { sion Number }\end{array}$ & Studies \\
\hline 463 & CGN & Q9P2M7 & I & 510 & CNN2 & Q99439 & $s, I$ \\
\hline 464 & CGNL1 & Q0VF96 & s & 511 & CNP & P09543 & 0 \\
\hline 465 & CHCHD10 & Q8WYQ3 & I & 512 & CNTN2 & Q02246 & s \\
\hline 466 & $\mathrm{CHCHD} 3$ & Q9NX63 & 0 & 513 & COASY & Q13057 & 1,0 \\
\hline 467 & CHD4 & Q14839 & 0 & 514 & COG5 & Q9UP83 & o \\
\hline 468 & $\mathrm{CHDH}$ & Q8NE62 & 0 & 515 & COG7 & P83436 & I \\
\hline 469 & CHI3L2 & Q15782 & s & 516 & COG8 & Q96MW5 & 1 \\
\hline 470 & CHL1 & 000533 & I & 517 & COL1A1 & P02452 & $\mathrm{a}$ \\
\hline 471 & CHMP4B & Q9H444 & I & 518 & COL1A2 & P08123 & $0, a$ \\
\hline 472 & CHMP5 & Q9NZZ3 & I & 519 & COL28A1 & Q2UY09 & s \\
\hline 473 & CHORDC1 & Q9UHD1 & I & 520 & COL4A3BP & Q9Y5P4 & 1 \\
\hline 474 & CHP1 & Q99653 & 0 & 521 & COL5A3 & P25940 & s \\
\hline 475 & CHTOP & Q9Y3Y2 & o & 522 & COL7A1 & Q02388 & o \\
\hline 476 & CIAO1 & 076071 & I & 523 & COMMD3 & Q9UBI1 & 1 \\
\hline 477 & CIB1 & Q99828 & $\mathrm{l}, \mathrm{h}$ & 524 & COMMD9 & Q9P000 & 1 \\
\hline 478 & CIRBP & Q14011 & I & 525 & COMT & P21964 & I \\
\hline 479 & CISD1 & Q9NZ45 & 0,1 & 526 & COPA & P53621 & o,l \\
\hline 480 & CKAP4 & Q07065 & 0,1 & 527 & СOPB1 & P53618 & 0,1 \\
\hline 481 & CKAP5 & Q14008 & s & 528 & COPB2 & P35606 & 0 \\
\hline 482 & CKB & P12277 & $\mathrm{s}, \mathrm{r}, \mathrm{c}, \mathrm{l}, \mathrm{p}, \mathrm{O}$ & 529 & COPE & 014579 & I \\
\hline 483 & CKMT1A & P12532 & $\mathrm{I}, \mathrm{O}$ & 530 & COPG1 & H0Y8X7 & I \\
\hline 484 & CKMT1B & P12532 & $\mathrm{I}, \mathrm{O}$ & 531 & COPG2 & Q9UBF2 & I \\
\hline 485 & $\mathrm{CLC}$ & Q05315 & $\mathrm{l}, \mathrm{t}, \mathrm{p}, \mathrm{t}$ & 532 & COPS2 & P61201 & I \\
\hline 486 & CLDN1 & 095832 & 0,1 & 533 & COPS4 & Q9BT78 & $h, l$ \\
\hline 487 & CLDN3 & 015551 & 0,1 & 534 & COPS5 & Q92905 & 1 \\
\hline 488 & CLDN4 & 014493 & I & 535 & COPS6 & Q7L5N1 & I \\
\hline 489 & CLDN6 & P56747 & I & 536 & COPS7A & Q9UBW8 & I \\
\hline 490 & CLDN7 & 095471 & I & 537 & COPS8 & Q99627 & I \\
\hline 491 & CLDN9 & 095484 & I & 538 & COPZ1 & F8VXR1 & 1 \\
\hline 492 & CLEC3B & P05452 & I & 539 & COQ6 & Q9Y2Z9 & s \\
\hline 493 & CLEC4F & Q8N1N0 & $\mathrm{k}$ & 540 & COR01A & P31146 & $s, r, o, I$ \\
\hline 494 & CLIC1 & O00299 & $c, r, s, o, p, I$ & 541 & CORO1B & Q9BR76 & 1 \\
\hline 495 & CLIC6 & Q96NY7 & 0,1 & 542 & CORO1C & Q9ULV4 & 1 \\
\hline 496 & CLINT1 & HOYCL3 & $\mathrm{I}, \mathrm{O}$ & 543 & CORO7 & P57737 & I \\
\hline 497 & CLIP1 & P30622 & I & 544 & COTL1 & Q14019 & I \\
\hline 498 & CLMN & Q96JQ2 & I & 545 & COX4I1 & P13073 & $\mathrm{I}, \mathrm{O}$ \\
\hline 499 & CLTA & P09496 & $\mathrm{I}, \mathrm{O}$ & 546 & $\operatorname{cox} 5 A$ & P20674 & $\mathrm{I}, \mathrm{O}$ \\
\hline 500 & CLTC & Q00610 & $\mathrm{r}, \mathrm{s}, \mathrm{l}, \mathrm{o}$ & 547 & COX5B & P10606 & 1,0 \\
\hline 501 & CLU & P10909 & $b, s, r, k, o, l$ & 548 & COX6A1 & P12074 & 1 \\
\hline 502 & CLUH & 075153 & I & 549 & COX6B1 & P14854 & I \\
\hline 503 & CMAS & Q8NFW8 & I & 550 & сох6C & P09669 & 0 \\
\hline 504 & CMBL & Q96DG6 & I & 551 & COX7A2 & P14406 & $\mathrm{I}, \mathrm{O}$ \\
\hline 505 & CMPK1 & P30085 & $j, o, l, m, j$ & 552 & COX7B & P24311 & o \\
\hline 506 & CMPK2 & Q5EBM0 & I & 553 & cox7C & P15954 & o \\
\hline 507 & CNBP & P62633 & I & 554 & $\mathrm{CP}$ & P00450 & $s, r, i, o, l$ \\
\hline 508 & CNDP2 & Q96KP4 & $\mathrm{s}, \mathrm{r}, \mathrm{l}, \mathrm{O}$ & 555 & CPB2 & Q96IY4 & I \\
\hline 509 & CNN1 & P51911 & 0 & 556 & CPD & 075976 & 0 \\
\hline
\end{tabular}




\begin{tabular}{|c|c|c|c|c|c|c|c|}
\hline & Gene & $\begin{array}{l}\text { UniProt Acces- } \\
\text { sion Number }\end{array}$ & Studies & & Gene & $\begin{array}{l}\text { UniProt Acces- } \\
\text { sion Number }\end{array}$ & Studies \\
\hline 557 & CPNE1 & Q99829 & 1 & 604 & CTSC & P53634 & 1 \\
\hline 558 & CPNE3 & 075131 & 1 & 605 & CTSD & P07339 & $\mathrm{s}, \mathrm{r}, \mathrm{o}, \mathrm{m}, \mathrm{I}$ \\
\hline 559 & CPPED1 & Q9BRF8 & I & 606 & CTSG & P08311 & $\mathrm{s}, \mathrm{r}, \mathrm{O}, \mathrm{l}$ \\
\hline 560 & CPS1 & P31327 & g & 607 & CTSH & P09668 & I \\
\hline 561 & CPSF3 & Q9UKF6 & I & 608 & CTSS & P25774 & I \\
\hline 562 & CPSF6 & Q16630 & I & 609 & CTSZ & Q9UBR2 & 1 \\
\hline 563 & CPSF7 & Q8N684 & I & 610 & CTTN & Q14247 & 0,1 \\
\hline 564 & CPT1A & P50416 & o & 611 & CUL1 & Q13616 & $\mathrm{s}, \mathrm{I}$ \\
\hline 565 & СРT2 & P23786 & $\mathrm{I}, \mathrm{O}$ & 612 & CUL2 & Q13617 & 1 \\
\hline 566 & CRABP2 & P29373 & I & 613 & CUL3 & Q13618 & I \\
\hline 567 & CRIP1 & P50238 & I & 614 & CUL5 & Q93034 & I \\
\hline 568 & CRIP2 & P52943 & I & 615 & CUTA & O60888 & 1 \\
\hline 569 & CRISP3 & P54108 & $r, s, l$ & 616 & CXCL1 & P09341 & I \\
\hline 570 & CRK & P46108 & I & 617 & CXCL17 & Q6UXB2 & $r$ \\
\hline 571 & CRKL & P46109 & I & 618 & CYB561 & P49447 & o \\
\hline 572 & CRNN & Q9UBG3 & I & 619 & CYB5A & P00167 & I \\
\hline 573 & CROCC & Q5TZA2 & 0,1 & 620 & CYB5B & J3QR91 & $\mathrm{l}, \mathrm{O}$ \\
\hline 574 & CROCCP3 & Q8IVE0 & 1 & 621 & CYB5R1 & Q9UHQ9 & I \\
\hline 575 & CROT & Q9UKG9 & I & 622 & CYB5R2 & Q6BCY4 & I \\
\hline 576 & CRYL1 & Q9Y2S2 & I & 623 & CYB5R3 & P00387 & 0,1 \\
\hline 577 & CRYM & Q14894 & 0,1 & 624 & CYBA & P13498 & o \\
\hline 578 & CRYZ & Q08257 & 0,1 & 625 & CYBB & P04839 & o \\
\hline 579 & CRYZL1 & 095825 & 1 & 626 & CYBC1 & Q9BQA9 & o \\
\hline 580 & CS & 075390 & 0,1 & 627 & CYC1 & P08574 & 0,1 \\
\hline 581 & CSDE1 & O75534 & 1 & 628 & CYCS & P99999 & $\mathrm{I}, \mathrm{O}$ \\
\hline 582 & CSE1L & P55060 & $s, 1$ & 629 & CYFIP1 & Q7L576 & 0,1 \\
\hline 583 & CSF1 & P09603 & $\mathrm{h}$ & 630 & CYP1B1 & Q16678 & o \\
\hline 584 & CSK & P41240 & I & 631 & CYP2A6 & P11509 & o \\
\hline 585 & CSNK1A1 & P48729 & I & 632 & CYP2J2 & P51589 & o \\
\hline 586 & CSNK2A1 & P68400 & I & 633 & CYP2S1 & Q96SQ9 & 0,1 \\
\hline 587 & CSRP1 & P21291 & I & 634 & CYP3A43 & Q9HB55 & a \\
\hline 588 & CSRP2 & Q16527 & 1 & 635 & CYP4B1 & P13584 & o \\
\hline 589 & CST1 & P01037 & $f, c, s, k, l$ & 636 & CYP4F11 & Q9HBI6 & o \\
\hline 590 & CST3 & P01034 & $f, s, r, k, l$ & 637 & CYP4F12 & Q9HCS2 & o \\
\hline 591 & CST4 & P01036 & $\mathrm{s}, \mathrm{r}, \mathrm{k}, \mathrm{l}, \mathrm{a}$ & 638 & CYP4F2 & P78329 & o \\
\hline 592 & CSTA & P01040 & $\mathrm{f}, \mathrm{l}, \mathrm{a}$ & 639 & CZIB & Q9NWV4 & I \\
\hline 593 & CSTB & P04080 & $s, r, k, o, l, a$ & 640 & DAD1 & P61803 & o \\
\hline 594 & СТВP1 & Q13363 & I & 641 & DAG1 & Q14118 & 1 \\
\hline 595 & СТВР2 & P56545 & I & 642 & DARS & P14868 & $\mathrm{I}, \mathrm{O}$ \\
\hline 596 & CTNNA1 & E5RJP7 & $\mathrm{I}, \mathrm{O}$ & 643 & DAW1 & Q8N136 & I \\
\hline 597 & CTNNA2 & P26232 & I & 644 & DAZAP1 & Q96EP5 & 1 \\
\hline 598 & CTNNB1 & P35222 & 0,1 & 645 & DBI & P07108 & $r, s, l$ \\
\hline 599 & CTNNBL1 & Q8WYA6 & I & 646 & DBNL & Q9UJU6 & $\mathrm{I}, \mathrm{O}$ \\
\hline 600 & CTNND1 & C9JZR2 & $\mathrm{I}, \mathrm{O}$ & 647 & DCD & P81605 & $\mathrm{s}, \mathrm{r}, \mathrm{o}, \mathrm{a}$ \\
\hline 601 & CTPS1 & P17812 & I & 648 & DCPS & Q96C86 & I \\
\hline 602 & CTPS2 & Q9NRF8 & I & 649 & DCTD & P32321 & 1 \\
\hline 603 & CTSB & P07858 & $\mathrm{r}, \mathrm{o}, \mathrm{m}, \mathrm{h}, \mathrm{I}$ & 650 & DCTN1 & Q14203 & I \\
\hline
\end{tabular}




\begin{tabular}{|c|c|c|c|c|c|c|c|}
\hline & Gene & $\begin{array}{l}\text { UniProt Acces- } \\
\text { sion Number }\end{array}$ & Studies & & Gene & $\begin{array}{l}\text { UniProt Acces- } \\
\text { sion Number }\end{array}$ & Studies \\
\hline 651 & DCTN2 & Q13561 & I & 698 & DIDO1 & Q9BTC0 & 0 \\
\hline 652 & DCTN4 & Q9UJW0 & I & 699 & DIS3 & Q9Y2L1 & I \\
\hline 653 & DCUN1D1 & Q96GG9 & I & 700 & DLAT & P10515 & 0,1 \\
\hline 654 & DCXR & Q7Z4W1 & I & 701 & DLD & P09622 & 1,0 \\
\hline 655 & DDAH1 & 094760 & I & 702 & DLST & P36957 & 0,1 \\
\hline 656 & $\mathrm{DDAH} 2$ & 095865 & I & 703 & DMBT1 & Q9UGM3 & $\mathrm{r}, \mathrm{s}, \mathrm{k}, \mathrm{l}, \mathrm{O}$ \\
\hline 657 & DDB1 & Q16531 & $\mathrm{I}, \mathrm{O}$ & 704 & DMTF1 & Q9Y222 & $\mathrm{h}$ \\
\hline 658 & DDI2 & Q5TDH0 & I & 705 & DNAH5 & Q8TE73 & 1,0 \\
\hline 659 & DDOST & P39656 & $\mathrm{I}, \mathrm{O}$ & 706 & DNAH8 & Q96JB1 & 0 \\
\hline 660 & DDRGK1 & Q96HY6 & 0,1 & 707 & DNAH9 & Q9NYC9 & $\mathrm{I}, \mathrm{O}$ \\
\hline 661 & DDTL & A6NHG4 & I & 708 & DNAI1 & Q9UI46 & I \\
\hline 662 & DDX1 & Q92499 & I & 709 & DNAI2 & Q9GZS0 & 1 \\
\hline 663 & DDX17 & Q92841 & 0,1 & 710 & DNAJA1 & P31689 & 1 \\
\hline 664 & DDX18 & Q9NVP1 & 0 & 711 & DNAJA2 & O60884 & 1 \\
\hline 665 & DDX19B & Q9UMR2 & I & 712 & DNAJA3 & Q96EY1 & 0 \\
\hline 666 & DDX21 & Q9NR30 & o & 713 & DNAJA4 & Q8WW22 & I \\
\hline 667 & DDX39A & 000148 & o & 714 & DNAJB1 & P25685 & I \\
\hline 668 & DDX39B & Q13838 & I & 715 & DNAJB12 & Q9NXW2 & o \\
\hline 669 & DDX3X & 000571 & $\mathrm{I}, \mathrm{O}$ & 716 & DNAJB13 & P59910 & I \\
\hline 670 & DDX42 & Q86XP3 & I & 717 & DNAJB6 & 075190 & I \\
\hline 671 & DDX46 & Q7L014 & I & 718 & DNAJC11 & Q9NVH1 & $\mathrm{I}, \mathrm{O}$ \\
\hline 672 & DDX5 & P17844 & 0,1 & 719 & DNAJC13 & 075165 & 0 \\
\hline 673 & DDX51 & Q8N8A6 & o & 720 & DNAJC2 & Q99543 & I \\
\hline 674 & DDX58 & 095786 & I & 721 & DNAJC8 & O75937 & I \\
\hline 675 & DDX6 & P26196 & 0,1 & 722 & DNAL1 & Q4LDG9 & 1 \\
\hline 676 & DECR1 & Q16698 & 0,1 & 723 & DNALI1 & 014645 & I \\
\hline 677 & DEFA1 & P59665 & $\mathrm{s}, \mathrm{O}, \mathrm{a}$ & 724 & DNM1L & 000429 & I \\
\hline 678 & DEFA1B & P59665 & $\mathrm{s}, \mathrm{O}, \mathrm{a}$ & 725 & DNM2 & P50570 & I \\
\hline 679 & DEFA3 & P59666 & $r, k$ & 726 & DNPEP & Q9ULA0 & 1 \\
\hline 680 & DERL1 & Q9BUN8 & o & 727 & DNPH1 & O43598 & 1 \\
\hline 681 & DES & P17661 & $m, o$ & 728 & $\mathrm{DOHH}$ & Q9BU89 & I \\
\hline 682 & DFFA & $\mathrm{O} 00273$ & I & 729 & DPCD & Q9BVM2 & 1 \\
\hline 683 & DGAT1 & 075907 & 0 & 730 & DPP3 & Q9NY33 & I \\
\hline 684 & DGKA & P23743 & I & 731 & DPP7 & Q9UHL4 & I \\
\hline 685 & DGLUCY & Q7Z3D6 & I & 732 & DPY30 & Q9C005 & I \\
\hline 686 & DHCR7 & Q9UBM7 & 0 & 733 & DPYS & Q14117 & I \\
\hline 687 & $\mathrm{DHODH}$ & Q02127 & 0 & 734 & DPYSL2 & Q16555 & I \\
\hline 688 & DHRS1 & Q96LJ7 & 1 & 735 & DRG1 & Q9Y295 & I \\
\hline 689 & DHRS3 & 075911 & 0 & 736 & DRG2 & P55039 & 1 \\
\hline 690 & DHRS7 & Q9Y394 & 0,1 & 737 & DSG1 & Q02413 & $\mathrm{a}$ \\
\hline 691 & DHRS7B & Q6IAN0 & I & 738 & DSG2 & Q14126 & 0,1 \\
\hline 692 & DHRS9 & Q9BPW9 & $\mathrm{I}, \mathrm{O}$ & 739 & DSG3 & P32926 & 0,1 \\
\hline 693 & DHX15 & 043143 & 0,1 & 740 & DSP & P15924 & $\mathrm{I}, \mathrm{O}, \mathrm{a}$ \\
\hline 694 & DHX30 & Q7L2E3 & o & 741 & DSTN & P60981 & $\mathrm{s}, \mathrm{l}$ \\
\hline 695 & DHX9 & Q08211 & 0,1 & 742 & DTX3L & Q8TDB6 & 1 \\
\hline 696 & DIABLO & Q9NR28 & $\mathrm{I}, \mathrm{I}$ & 743 & DUOX1 & Q9NRD9 & 0 \\
\hline 697 & DIAPH1 & 060610 & I & 744 & DUOX2 & Q9NRD8 & 0 \\
\hline
\end{tabular}




\begin{tabular}{|c|c|c|c|c|c|c|c|}
\hline & Gene & $\begin{array}{l}\text { UniProt Acces- } \\
\text { sion Number }\end{array}$ & Studies & & Gene & $\begin{array}{l}\text { UniProt Acces- } \\
\text { sion Number }\end{array}$ & Studies \\
\hline 745 & DUSP23 & Q9BVJ7 & 1 & 792 & EIF2S3 & P41091 & 1 \\
\hline 746 & DYNC1H1 & Q14204 & $\mathrm{l}, \mathrm{O}$ & 793 & EIF3A & Q14152 & $\mathrm{l}, \mathrm{O}$ \\
\hline 747 & DYNC1/2 & Q13409 & $\mathrm{l}, \mathrm{O}$ & 794 & EIF3B & P55884 & I \\
\hline 748 & DYNC1LI1 & Q9Y6G9 & I & 795 & EIF3C & Q99613 & 0,1 \\
\hline 749 & DYNC1LI2 & O43237 & I & 796 & EIF3D & 015371 & I \\
\hline 750 & DYNC2H1 & Q8NCM8 & I & 797 & EIF3E & P60228 & 1 \\
\hline 751 & DYNLL1 & P63167 & I & 798 & EIF3F & 000303 & I \\
\hline 752 & DYNLRB1 & Q9NP97 & I & 799 & EIF3G & O75821 & 1 \\
\hline 753 & DYNLRB2 & Q8TF09 & 1 & 800 & EIF3H & 015372 & 1 \\
\hline 754 & DYNLT1 & P63172 & 1 & 801 & EIF3I & Q13347 & $\mathrm{l}, \mathrm{h}$ \\
\hline 755 & DYSF & O75923 & o & 802 & EIF3K & Q9UBQ5 & I \\
\hline 756 & EBP & Q15125 & I & 803 & EIF3L & Q9Y262 & 1 \\
\hline 757 & ECE1 & P42892 & o & 804 & EIF3M & Q7L2H7 & I \\
\hline 758 & $\mathrm{ECH} 1$ & Q13011 & $\mathrm{I}, \mathrm{O}$ & 805 & EIF4A1 & P60842 & $\mathrm{s}, \mathrm{O}, \mathrm{l}$ \\
\hline 759 & $\mathrm{ECHDC1}$ & Q9NTX5 & 1 & 806 & EIF4A2 & Q14240 & 1 \\
\hline 760 & ECHS1 & P30084 & $0, j, m, l, j$ & 807 & EIF4A3 & P38919 & 0,1 \\
\hline 761 & ECl1 & P42126 & 0,1 & 808 & EIF4B & P23588 & 0,1 \\
\hline 762 & ECPAS & Q5VYK3 & I & 809 & EIF4E & P06730 & I \\
\hline 763 & EDC4 & Q6P2E9 & I & 810 & EIF4G1 & Q04637 & $\mathrm{l}, \mathrm{O}$ \\
\hline 764 & EDF1 & 060869 & I & 811 & EIF4G2 & P78344 & o \\
\hline 765 & EEA1 & Q15075 & I & 812 & EIF4H & Q15056 & 1 \\
\hline 766 & EEF1A1 & P68104 & $\mathrm{s}, \mathrm{O}, \mathrm{a}$ & 813 & EIF5 & P55010 & I \\
\hline 767 & EEF1A1P5 & Q5VTE0 & $r, l$ & 814 & EIF5A & P63241 & $r, l$ \\
\hline 768 & EEF1B2 & P24534 & $\mathrm{l}, \mathrm{O}$ & 815 & EIF5B & 060841 & I \\
\hline 769 & EEF1D & P29692 & $\mathrm{I}, \mathrm{O}$ & 816 & EIF6 & P56537 & I \\
\hline 770 & EEF1E1 & O43324 & I & 817 & ELANE & P08246 & $\mathrm{s}, \mathrm{r}, \mathrm{k}, \mathrm{o}, \mathrm{l}$ \\
\hline 771 & EEF1G & P26641 & I & 818 & ELAVL1 & Q15717 & $\mathrm{l}, \mathrm{O}$ \\
\hline 772 & EEF2 & P13639 & $\mathrm{s}, \mathrm{r}, \mathrm{l}, \mathrm{O}$ & 819 & ELMOD2 & Q8IZ81 & o \\
\hline 773 & EFCAB1 & Q9HAE3 & I & 820 & ELOB & Q15370 & 1 \\
\hline 774 & EFHC1 & Q5JVL4 & I & 821 & EMC1 & Q8N766 & o \\
\hline 775 & EFHC2 & Q5JST6 & I & 822 & EMC3 & Q9P012 & o \\
\hline 776 & EFHD2 & Q96C19 & 1 & 823 & EMC6 & Q9BV81 & o \\
\hline 777 & EFTUD2 & Q15029 & I & 824 & EMC7 & Q9NPA0 & o \\
\hline 778 & EGFR & P00533 & o & 825 & EMD & P50402 & 0,1 \\
\hline 779 & EHD2 & Q9NZN4 & $\mathrm{h}$ & 826 & EML1 & 000423 & I \\
\hline 780 & EHD3 & Q9NZN3 & I & 827 & EML2 & O95834 & I \\
\hline 781 & EHD4 & Q9H223 & 0,1 & 828 & EML4 & Q9HC35 & I \\
\hline 782 & EHHADH & Q08426 & o & 829 & EMP2 & P54851 & c \\
\hline 783 & EIF1 & P41567 & I & 830 & ENDOG & Q14249 & I \\
\hline 784 & EIF1AY & 014602 & I & 831 & ENO1 & P06733 & $c, s, r, r, n, m, l, o, a$ \\
\hline 785 & EIF2A & Q9BY44 & I & 832 & ENO2 & P09104 & $h, l$ \\
\hline 786 & EIF2AK2 & P19525 & I & 833 & ENOPH 1 & Q9UHY7 & 1 \\
\hline 787 & EIF2B1 & Q14232 & I & 834 & ENSA & 043768 & 1 \\
\hline 788 & EIF2B2 & P49770 & I & 835 & ENTPD3 & 075355 & o \\
\hline 789 & EIF2B3 & Q9NR50 & I & 836 & EPB41L1 & Q9H4G0 & 0,1 \\
\hline 790 & EIF2S1 & P05198 & $\mathrm{l}, \mathrm{d}$ & 837 & EPB42 & P16452 & o \\
\hline 791 & EIF2S2 & P20042 & I & 838 & EPCAM & P16422 & $\mathrm{I}, \mathrm{O}$ \\
\hline
\end{tabular}




\begin{tabular}{|c|c|c|c|c|c|c|c|}
\hline & Gene & $\begin{array}{l}\text { UniProt Acces- } \\
\text { sion Number }\end{array}$ & Studies & & Gene & $\begin{array}{l}\text { UniProt Acces- } \\
\text { sion Number }\end{array}$ & Studies \\
\hline 839 & EPHA2 & P29317 & o & 886 & FAM107B & Q9H098 & I \\
\hline 840 & EPHX1 & P07099 & 0,1 & 887 & FAM114A1 & Q8IWE2 & I \\
\hline 841 & EPHX2 & P34913 & I & 888 & FAM120A & Q9NZB2 & 0,1 \\
\hline 842 & EPM2AIP1 & Q7L775 & I & 889 & FAM162A & Q96A26 & 0 \\
\hline 843 & EPPK1 & P58107 & $\mathrm{s}, \mathrm{l}, \mathrm{o}$ & 890 & FAM169A & Q9Y6X4 & s \\
\hline 844 & EPRS & P07814 & $\mathrm{I}, \mathrm{O}$ & 891 & FAM174A & Q8TBP5 & 0 \\
\hline 845 & EPS8 & Q12929 & I & 892 & FAM3C & Q92520 & 0 \\
\hline 846 & EPS8L2 & Q9H6S3 & I & 893 & FAM3D & Q96BQ1 & 1,0 \\
\hline 847 & EPX & P11678 & 0 & 894 & FAM49B & Q9NUQ9 & I \\
\hline 848 & ERAP1 & Q9NZ08 & 0,1 & 895 & FAM81B & Q96LP2 & $\mathrm{I}, \mathrm{O}$ \\
\hline 849 & ERBB2 & P04626 & 0 & 896 & FAM98C & Q17RN3 & 1 \\
\hline 850 & ERGIC1 & Q969X5 & $\mathrm{I}, \mathrm{O}$ & 897 & FANCD2 & Q9BXW9 & I \\
\hline 851 & ERH & P84090 & o & 898 & FARSA & Q9Y285 & 1 \\
\hline 852 & ERICH3 & Q5RHP9 & I & 899 & FARSB & Q9NSD9 & 1 \\
\hline 853 & ERICH5 & Q6P6B1 & 0 & 900 & FASN & P49327 & o,l \\
\hline 854 & ERLIN1 & 075477 & 0 & 901 & FAT3 & Q8TDW7 & I \\
\hline 855 & ERLIN2 & 094905 & 1,0 & 902 & FAU & P62861 & I \\
\hline 856 & ERMP1 & Q7Z2K6 & 0 & 903 & FBL & P22087 & 0,1 \\
\hline 857 & ERN2 & Q76MJ5 & 0 & 904 & FBLN1 & P23142 & I \\
\hline 858 & ERO1A & Q96HE7 & 0,1 & 905 & FBP1 & P09467 & $\mathrm{s}, \mathrm{O}, \mathrm{I}$ \\
\hline 859 & ERP29 & P30040 & $m, o, l$ & 906 & FBXL4 & Q9UKA2 & I \\
\hline 860 & ERP44 & Q9BS26 & 0,1 & 907 & FBXO2 & Q9UK22 & I \\
\hline 861 & ESD & P10768 & $\mathrm{I}, \mathrm{p}$ & 908 & $\mathrm{FBXO} 22$ & Q8NEZ5 & I \\
\hline 862 & ESYT1 & Q9BSJ8 & 1,0 & 909 & FBXW9 & Q5XUX1 & I \\
\hline 863 & ESYT2 & A0FGR8 & 0 & 910 & FCGBP & Q9Y6R7 & $s, r, k, o, l$ \\
\hline 864 & ETF1 & P62495 & I & 911 & FCGR3B & 075015 & I \\
\hline 865 & ETFA & P13804 & $\mathrm{I}, \mathrm{O}$ & 912 & FCSK & Q8N0W3 & I \\
\hline 866 & ETFB & P38117 & I & 913 & FDFT1 & P37268 & 0 \\
\hline 867 & ETFDH & Q16134 & 0,1 & 914 & FDPS & P14324 & 1 \\
\hline 868 & ETHE1 & 095571 & 0,1 & 915 & FDXR & P22570 & 0,1 \\
\hline 869 & EVPL & Q92817 & 0,1 & 916 & FECH & P22830 & o \\
\hline 870 & EWSR1 & Q01844 & 0,1 & 917 & FEN1 & P39748 & 1 \\
\hline 871 & EXD2 & Q9NVHO & 0 & 918 & FER1L6 & Q2WGJ9 & 1 \\
\hline 872 & EXOC1 & Q9NV70 & I & 919 & FERMT3 & Q86UX7 & I \\
\hline 873 & EXOC2 & Q96KP1 & I & 920 & FGA & P02671 & $s, r, l, o$ \\
\hline 874 & EZR & P15311 & $s, r, o, l$ & 921 & FGB & P02675 & $b, s, r, k, l, o$ \\
\hline 875 & F11R & Q9Y624 & 0 & 922 & FGG & P02679 & $s, r, l, j, o, j$ \\
\hline 876 & F12 & P00748 & I & 923 & $\mathrm{FH}$ & P07954 & 0,1 \\
\hline 877 & $\mathrm{~F} 13 \mathrm{~B}$ & P05160 & I & 924 & FHAD1 & B1AJZ9 & 1 \\
\hline 878 & $\mathrm{~F} 2$ & P00734 & $r, l$ & 925 & FHIT & P49789 & I \\
\hline 879 & F3 & P13726 & 0,1 & 926 & FIS1 & Q9Y3D6 & 1 \\
\hline 880 & FABP1 & P07148 & $g$ & 927 & FKBP11 & Q9NYL4 & 0 \\
\hline 881 & FABP4 & P15090 & c & 928 & FKBP1A & P62942 & $s, 1$ \\
\hline 882 & FABP5 & Q01469 & $r, s, n, u, k, o, l, p, a$ & 929 & FKBP2 & P26885 & I \\
\hline 883 & FAF2 & Q96CS3 & o & 930 & FKBP3 & Q00688 & 1 \\
\hline 884 & $\mathrm{FAH}$ & P16930 & I & 931 & FKBP4 & Q02790 & I \\
\hline 885 & FAHD1 & Q6P587 & I & 932 & FKBP5 & Q13451 & I \\
\hline
\end{tabular}




\begin{tabular}{|c|c|c|c|c|c|c|c|}
\hline & Gene & $\begin{array}{l}\text { UniProt Acces- } \\
\text { sion Number }\end{array}$ & Studies & & Gene & $\begin{array}{l}\text { UniProt Acces- } \\
\text { sion Number }\end{array}$ & Studies \\
\hline 933 & FKBP8 & Q14318 & o & 980 & GBP5 & Q96PP8 & 1 \\
\hline 934 & FLAD1 & Q8NFF5 & 1 & 981 & GBP6 & Q6ZN66 & 1 \\
\hline 935 & FLG & P20930 & $r, a$ & 982 & GC & P02774 & $r, s, u, k, c, l$ \\
\hline 936 & FLII & Q13045 & 1 & 983 & GCA & P28676 & 1 \\
\hline 937 & FLNA & P21333 & 0,1 & 984 & GCHFR & P30047 & 1 \\
\hline 938 & FLNB & 075369 & 0,1 & 985 & GCLC & P48506 & 0,1 \\
\hline 939 & FLOT1 & 075955 & $\mathrm{l}, \mathrm{O}$ & 986 & GCLM & P48507 & I \\
\hline 940 & FLOT2 & Q14254 & o & 987 & GCN1 & Q92616 & 0,1 \\
\hline 941 & FMC1 & Q96HJ9 & I & 988 & GDAP1 & Q8TB36 & 0 \\
\hline 942 & FMO3 & P31513 & $\mathrm{l}, \mathrm{O}$ & 989 & GDI1 & P31150 & I \\
\hline 943 & FN1 & P02751 & 0,1 & 990 & GDI2 & P50395 & $r, s, o, l, p$ \\
\hline 944 & FNTA & P49354 & I & 991 & GDPD3 & Q7L5L3 & o \\
\hline 945 & FOXO3 & O43524 & I & 992 & GDPGP1 & Q6ZNW5 & 1 \\
\hline 946 & FSCN1 & Q16658 & 1 & 993 & GFAP & P14136 & $b$ \\
\hline 947 & FTH1 & P02794 & I & 994 & GFPT1 & Q06210 & 0,1 \\
\hline 948 & FTL & P02792 & I & 995 & GGCT & 075223 & I \\
\hline 949 & FTO & Q9C0B1 & I & 996 & GGPS1 & 095749 & I \\
\hline 950 & FUBP1 & Q96AE4 & 0,1 & 997 & $\mathrm{GH} 1$ & P01241 & s \\
\hline 951 & FUCA1 & P04066 & I & 998 & GHITM & Q9H3K2 & o \\
\hline 952 & FUS & P35637 & 0,1 & 999 & GIPC1 & 014908 & 1 \\
\hline 953 & FUT2 & Q10981 & o & 1000 & GK3P & Q14409 & 1 \\
\hline 954 & FUT3 & P21217 & 0,1 & 1001 & GLA & P06280 & $\mathrm{l}, \mathrm{h}$ \\
\hline 955 & FUT6 & P51993 & 0,1 & 1002 & GLB1 & P16278 & 0,1 \\
\hline 956 & FUT8 & Q9BYC5 & o & 1003 & GLCE & 094923 & o \\
\hline 957 & G3BP1 & Q13283 & $\mathrm{l}, \mathrm{O}$ & 1004 & GLG1 & Q92896 & 0,1 \\
\hline 958 & G6PD & P11413 & $r, l$ & 1005 & GLIPR2 & Q9H4G4 & 0 \\
\hline 959 & GAA & P10253 & 0,1 & 1006 & GLO1 & Q04760 & $\mathrm{l}, \mathrm{j}$ \\
\hline 960 & GALE & Q14376 & I & 1007 & GLOD4 & Q9HC38 & I \\
\hline 961 & GALK1 & P51570 & I & 1008 & GLRX & P35754 & 1 \\
\hline 962 & GALM & Q96C23 & I & 1009 & GLRX3 & 076003 & I \\
\hline 963 & GALNT1 & Q10472 & o & 1010 & GLTP & Q9NZD2 & I \\
\hline 964 & GALNT12 & Q8IXK2 & o & 1011 & GLUD1 & P00367 & $c, 0, l$ \\
\hline 965 & GALNT4 & Q8N4A0 & o & 1012 & GLUD2 & P49448 & $\mathrm{m}$ \\
\hline 966 & GALNT5 & Q7Z7M9 & o & 1013 & GLUL & P15104 & 0,1 \\
\hline 967 & GALNT6 & Q8NCL4 & I & 1014 & GM2A & P17900 & I \\
\hline 968 & GALNT7 & Q86SF2 & o & 1015 & GMDS & O60547 & 1 \\
\hline 969 & GANAB & Q14697 & 0,1 & 1016 & GMFB & P60983 & I \\
\hline 970 & GAPDH & P04406 & $\mathrm{s}, \mathrm{r}, \mathrm{c}, \mathrm{o}, \mathrm{l}, \mathrm{p}, \mathrm{t}, \mathrm{a}, \mathrm{t}$ & 1017 & GMFG & 060234 & 1 \\
\hline 971 & GAPVD1 & Q14C86 & I & 1018 & GMPPA & Q96IJ6 & I \\
\hline 972 & GAR1 & Q9NY12 & o & 1019 & GMPPB & Q9Y5P6 & 1 \\
\hline 973 & GARS & P41250 & $\mathrm{l}, \mathrm{O}$ & 1020 & GMPR & P36959 & 1 \\
\hline 974 & GART & P22102 & 1 & 1021 & GMPR2 & Q9P2T1 & 1 \\
\hline 975 & GATD3A & PODPI2 & 0,1 & 1022 & GMPS & P49915 & 1 \\
\hline 976 & GBE1 & Q04446 & I & 1023 & GNA11 & P29992 & 0,1 \\
\hline 977 & GBP1 & P32455 & I & 1024 & GNAI1 & P63096 & o \\
\hline 978 & GBP2 & P32456 & I & 1025 & GNAI2 & P04899 & 0,1 \\
\hline 979 & GBP4 & Q96PP9 & I & 1026 & GNAI3 & P08754 & o \\
\hline
\end{tabular}




\begin{tabular}{|c|c|c|c|c|c|c|c|}
\hline & Gene & $\begin{array}{l}\text { UniProt Acces- } \\
\text { sion Number }\end{array}$ & Studies & & Gene & $\begin{array}{l}\text { UniProt Acces- } \\
\text { sion Number }\end{array}$ & Studies \\
\hline 1027 & GNAS & Q5JWF2 & o & 1074 & GSTM3 & P21266 & $\mathrm{I}, \mathrm{h}$ \\
\hline 1028 & GNB1 & P62873 & 0,1 & 1075 & GSTM4 & Q03013 & I \\
\hline 1029 & GNB2 & P62879 & I & 1076 & GSTO1 & P78417 & 0,1 \\
\hline 1030 & GNE & Q9Y223 & I & 1077 & GSTP1 & P09211 & $c, s, r, e, m, o, l, p, a, j$ \\
\hline 1031 & GNG12 & Q9UBI6 & o & 1078 & GSTT1 & P30711 & I \\
\hline 1032 & GNG13 & Q9P2W3 & $\mathrm{h}$ & 1079 & GSTZ1 & 043708 & I \\
\hline 1033 & GNG5 & P63218 & 0 & 1080 & GTF2I & P78347 & I \\
\hline 1034 & GNL1 & P36915 & I & 1081 & GTPBP1 & 000178 & I \\
\hline 1035 & GNPDA1 & P46926 & I & 1082 & GUSB & P08236 & I \\
\hline 1036 & GNPDA2 & Q8TDQ7 & I & 1083 & GYG1 & P46976 & I \\
\hline 1037 & GNPNAT1 & Q96EK6 & I & 1084 & GYS1 & P13807 & I \\
\hline 1038 & GNS & P15586 & I & 1085 & H1F0 & P07305 & 0,1 \\
\hline 1039 & GOLGB1 & Q14789 & 0,1 & 1086 & $\mathrm{H} 1 \mathrm{FX}$ & Q92522 & I \\
\hline 1040 & GOLM1 & Q8NBJ4 & 0,1 & 1087 & H2AFV & Q71UI9 & 1 \\
\hline 1041 & GOLPH3L & Q9H4A5 & I & 1088 & $\mathrm{H} 2 \mathrm{AFY}$ & 075367 & $\mathrm{I}, \mathrm{O}$ \\
\hline 1042 & GOLT1B & Q9Y3E0 & o & 1089 & $\mathrm{H} 2 \mathrm{AFY} 2$ & Q9P0M6 & I \\
\hline 1043 & GORASP2 & Q9H8Y8 & I & 1090 & $\mathrm{H} 2 \mathrm{AFZ}$ & P0C0S5 & s \\
\hline 1044 & GOSR1 & 095249 & 0 & 1091 & H3F3A & P84243 & s \\
\hline 1045 & GOT1 & P17174 & I & 1092 & H3F3B & P84243 & s \\
\hline 1046 & GOT2 & P00505 & $\mathrm{I}, \mathrm{O}$ & 1093 & H6PD & 095479 & I \\
\hline 1047 & GPAA1 & 043292 & 0 & 1094 & HACD3 & Q9P035 & I \\
\hline 1048 & GPD1L & Q8N335 & I & 1095 & $\mathrm{HADH}$ & Q16836 & $\mathrm{I}, \mathrm{O}$ \\
\hline 1049 & GPD2 & P43304 & 0,1 & 1096 & HADHA & P40939 & 0,1 \\
\hline 1050 & GPI & P06744 & $s, r, l, p, o$ & 1097 & HADHB & P55084 & 0,1 \\
\hline 1051 & GPNMB & Q14956 & I & 1098 & HAGH & Q16775 & 1 \\
\hline 1052 & GPR107 & Q5VW38 & 0 & 1099 & HAL & P42357 & $p$ \\
\hline 1053 & GPR89A & B7ZAQ6 & o & 1100 & HARS & P12081 & $\mathrm{I}, \mathrm{p}$ \\
\hline 1054 & GPS1 & Q13098 & I & 1101 & HBA1 & P69905 & $\mathrm{s}, \mathrm{r}, \mathrm{b}, \mathrm{c}, \mathrm{k}, \mathrm{o}, \mathrm{l}, \mathrm{a}$ \\
\hline 1055 & GPX1 & P07203 & 0,1 & 1102 & HBA2 & P69905 & $\mathrm{s}, \mathrm{r}, \mathrm{b}, \mathrm{c}, \mathrm{k}, \mathrm{o}, \mathrm{l}, \mathrm{a}$ \\
\hline 1056 & GPX2 & P18283 & I & 1103 & HBB & P68871 & s,r,b,c,q,p,m,o,e,l,q,a \\
\hline 1057 & GPX4 & P36969 & I & 1104 & $\mathrm{HBD}$ & P02042 & $\mathrm{s}, \mathrm{O}, \mathrm{l}$ \\
\hline 1058 & GRB2 & P62993 & I & 1105 & HBG1 & P69891 & o \\
\hline 1059 & GRHPR & Q9UBQ7 & I & 1106 & HCLS1 & P14317 & I \\
\hline 1060 & GRN & P28799 & $s, r, I$ & 1107 & HDAC1 & Q13547 & I \\
\hline 1061 & GSDMB & Q8TAX9 & I & 1108 & HDDC2 & Q7Z4H3 & I \\
\hline 1062 & GSDMD & P57764 & I & 1109 & HDDC3 & Q8N4P3 & I \\
\hline 1063 & GSN & P06396 & $\mathrm{s}, \mathrm{r}, \mathrm{O}, \mathrm{l}$ & 1110 & HDGF & P51858 & 0,1 \\
\hline 1064 & GSPT1 & P15170 & 1 & 1111 & HDGFL3 & Q9Y3E1 & 1 \\
\hline 1065 & GSR & P00390 & 0,1 & 1112 & HDHD2 & Q9H0R4 & I \\
\hline 1066 & GSS & P48637 & $\mathrm{I}, \mathrm{p}$ & 1113 & HDHD3 & Q9BSH5 & 1 \\
\hline 1067 & GSTA1 & P08263 & $\mathrm{S}, \mathrm{C}, \mathrm{l}, \mathrm{O}$ & 1114 & HDLBP & Q00341 & 0,1 \\
\hline 1068 & GSTA2 & P09210 & $\mathrm{r}, \mathrm{l}, \mathrm{e}$ & 1115 & HEATR1 & Q9H583 & I \\
\hline 1069 & GSTA3 & Q16772 & I & 1116 & HEBP1 & Q9NRV9 & I \\
\hline 1070 & GSTA5 & Q7RTV2 & I & 1117 & HEBP2 & Q9Y5Z4 & 0,1 \\
\hline 1071 & GSTK1 & Q9Y2Q3 & 0,1 & 1118 & HERC4 & Q5GLZ8 & I \\
\hline 1072 & GSTM1 & P09488 & I & 1119 & HEXA & P06865 & 1 \\
\hline 1073 & GSTM2 & P28161 & I & 1120 & HEXB & P07686 & $\mathrm{I}, \mathrm{O}$ \\
\hline
\end{tabular}




\begin{tabular}{|c|c|c|c|c|c|c|c|}
\hline & Gene & $\begin{array}{l}\text { UniProt Acces- } \\
\text { sion Number }\end{array}$ & Studies & & Gene & $\begin{array}{l}\text { UniProt Acces- } \\
\text { sion Number }\end{array}$ & Studies \\
\hline 1121 & HIBADH & P31937 & 0,1 & 1168 & HIST1H4L & P62805 & $\mathrm{b}, \mathrm{s}, \mathrm{r}, \mathrm{l}, \mathrm{o}, \mathrm{a}$ \\
\hline 1122 & $\mathrm{HIBCH}$ & Q6NVY1 & 1 & 1169 & HIST2H2AA3 & Q6FI13 & I \\
\hline 1123 & HID1 & Q8IV36 & o & 1170 & HIST2H2AA4 & Q6Fl13 & I \\
\hline 1124 & HINT1 & P49773 & I & 1171 & $\mathrm{HIST} 2 \mathrm{H} 2 \mathrm{AB}$ & Q8IUE6 & k \\
\hline 1125 & HINT2 & Q9BX68 & 0,1 & 1172 & HIST2H2AC & Q16777 & s \\
\hline 1126 & HIST1H1A & Q02539 & 0,1 & 1173 & HIST2H3A & Q71DI3 & $s, r$ \\
\hline 1127 & HIST1H1B & P16401 & 0,1 & 1174 & HIST2H3C & Q71DI3 & $s, r$ \\
\hline 1128 & HIST1H1C & P16403 & o & 1175 & HIST2H3D & Q71DI3 & $s, r$ \\
\hline 1129 & HIST1H1D & P16402 & $r$ & 1176 & HIST2H4A & P62805 & $\mathrm{b}, \mathrm{s}, \mathrm{r}, \mathrm{l}, \mathrm{o}, \mathrm{a}$ \\
\hline 1130 & HIST1H1E & P10412 & $\mathrm{s}, \mathrm{l}$ & 1177 & HIST2H4B & P62805 & $\mathrm{b}, \mathrm{s}, \mathrm{r}, \mathrm{l}, \mathrm{o}, \mathrm{a}$ \\
\hline 1131 & HIST1H2AA & Q96QV6 & o & 1178 & HIST3H2BB & Q8N257 & I \\
\hline 1132 & HIST1H2AB & P04908 & o & 1179 & HIST3H3 & Q16695 & o \\
\hline 1133 & HIST1H2AC & Q93077 & k & 1180 & HIST4H4 & P62805 & $\mathrm{b}, \mathrm{s}, \mathrm{r}, \mathrm{l}, \mathrm{o}, \mathrm{a}$ \\
\hline 1134 & HIST1H2AD & P20671 & $\mathrm{r}, \mathrm{o}$ & 1181 & HK1 & P19367 & 0,1 \\
\hline 1135 & HIST1H2AE & P04908 & o & 1182 & HK3 & P52790 & I \\
\hline 1136 & HIST1H2AH & Q96KK5 & s & 1183 & HLA-A & P01892 & $\mathrm{l}, \mathrm{I}, \mathrm{h}, \mathrm{I}$ \\
\hline 1137 & HIST1H2BA & Q96A08 & $b, o$ & 1184 & HLA-B & P01889 & $\mathrm{ol}, \mathrm{l}, \mathrm{h}$ \\
\hline 1138 & HIST1H2BB & P33778 & o & 1185 & HLA-C & P30510 & I \\
\hline 1139 & HIST1H2BC & P62807 & o & 1186 & HLA-DPA1 & P20036 & I \\
\hline 1140 & HIST1H2BE & P62807 & o & 1187 & HLA-DQB1 & P01920 & o \\
\hline 1141 & HIST1H2BF & P62807 & o & 1188 & HLA-DRA & P01903 & $\mathrm{l}, \mathrm{O}$ \\
\hline 1142 & HIST1H2BG & P62807 & o & 1189 & HLA-DRB1 & P13760 & I \\
\hline 1143 & HIST1H2BI & P62807 & o & 1190 & HM13 & Q8TCT9 & o \\
\hline 1144 & HIST1H2BJ & P06899 & s & 1191 & HMGA1 & P17096 & I \\
\hline 1145 & HIST1H2BK & O60814 & s & 1192 & HMGB1 & P09429 & $\mathrm{s}, \mathrm{l}$ \\
\hline 1146 & HIST1H2BL & Q99880 & I & 1193 & HMGB2 & P26583 & $s, 1$ \\
\hline 1147 & HIST1H2BM & Q99879 & $r$ & 1194 & HMGB3 & 015347 & I \\
\hline 1148 & HIST1H3A & P68431 & $\mathrm{s}, \mathrm{O}$ & 1195 & HMGCL & P35914 & 0,1 \\
\hline 1149 & HIST1H3B & P68431 & $\mathrm{s}, \mathrm{O}$ & 1196 & HMGCS2 & P54868 & 0,1 \\
\hline 1150 & HIST1H3C & P68431 & $\mathrm{s}, \mathrm{O}$ & 1197 & HMGN1 & P05114 & o \\
\hline 1151 & HIST1H3D & P68431 & $s, 0$ & 1198 & HMGN2 & P05204 & o \\
\hline 1152 & HIST1H3E & P68431 & $\mathrm{s}, \mathrm{O}$ & 1199 & HMGN5 & P82970 & I \\
\hline 1153 & HIST1H3F & P68431 & $\mathrm{s}, \mathrm{O}$ & 1200 & HMOX2 & P30519 & o \\
\hline 1154 & HIST1H3G & P68431 & $\mathrm{s}, \mathrm{O}$ & 1201 & HNMT & P50135 & I \\
\hline 1155 & HIST1H3H & P68431 & $\mathrm{s}, \mathrm{O}$ & 1202 & HNRNPAO & Q13151 & I \\
\hline 1156 & HIST1H3I & P68431 & $\mathrm{s}, \mathrm{O}$ & 1203 & HNRNPA1 & P09651 & $\mathrm{l}, \mathrm{O}$ \\
\hline 1157 & HIST1H3J & P68431 & $\mathrm{s}, \mathrm{O}$ & 1204 & HNRNPA1L2 & Q32P51 & s \\
\hline 1158 & HIST1H4A & P62805 & $\mathrm{b}, \mathrm{s}, \mathrm{r}, \mathrm{l}, \mathrm{o}, \mathrm{a}$ & 1205 & HNRNPA2B1 & P22626 & $\mathrm{r}, \mathrm{s}, \mathrm{o}, \mathrm{l}$ \\
\hline 1159 & HIST1H4B & P62805 & $\mathrm{b}, \mathrm{s}, \mathrm{r}, \mathrm{l}, \mathrm{o}, \mathrm{a}$ & 1206 & HNRNPA3 & P51991 & $\mathrm{s}, \mathrm{O}, \mathrm{l}$ \\
\hline 1160 & HIST1H4C & P62805 & $\mathrm{b}, \mathrm{s}, \mathrm{r}, \mathrm{l}, \mathrm{o}, \mathrm{a}$ & 1207 & HNRNPAB & Q99729 & I \\
\hline 1161 & HIST1H4D & P62805 & b,s,r,l,o,a & 1208 & HNRNPC & P07910 & $\mathrm{r}, \mathrm{s}, \mathrm{O}, \mathrm{l}$ \\
\hline 1162 & HIST1H4E & P62805 & $\mathrm{b}, \mathrm{s}, \mathrm{r}, \mathrm{l}, \mathrm{o}, \mathrm{a}$ & 1209 & HNRNPCL1 & O60812 & o \\
\hline 1163 & HIST1H4F & P62805 & $\mathrm{b}, \mathrm{s}, \mathrm{r}, \mathrm{l}, \mathrm{o}, \mathrm{a}$ & 1210 & HNRNPD & Q14103 & $\mathrm{r}, \mathrm{s}, \mathrm{O}, \mathrm{l}$ \\
\hline 1164 & HIST1H4H & P62805 & $\mathrm{b}, \mathrm{s}, \mathrm{r}, \mathrm{l}, \mathrm{o}, \mathrm{a}$ & 1211 & HNRNPDL & 014979 & I \\
\hline 1165 & HIST1H4I & P62805 & $\mathrm{b}, \mathrm{s}, \mathrm{r}, \mathrm{l}, \mathrm{o}, \mathrm{a}$ & 1212 & HNRNPF & P52597 & $\mathrm{l}, \mathrm{O}$ \\
\hline 1166 & HIST1H4J & P62805 & $\mathrm{b}, \mathrm{s}, \mathrm{r}, \mathrm{l}, \mathrm{o}, \mathrm{a}$ & 1213 & HNRNPH1 & P31943 & 0,1 \\
\hline 1167 & HIST1H4K & P62805 & $\mathrm{b}, \mathrm{s}, \mathrm{r}, \mathrm{l}, \mathrm{o}, \mathrm{a}$ & 1214 & HNRNPH2 & P55795 & I \\
\hline
\end{tabular}




\begin{tabular}{|c|c|c|c|c|c|c|c|}
\hline & Gene & $\begin{array}{l}\text { UniProt Acces- } \\
\text { sion Number }\end{array}$ & Studies & & Gene & $\begin{array}{l}\text { UniProt Acces- } \\
\text { sion Number }\end{array}$ & Studies \\
\hline 1215 & HNRNPH3 & P31942 & 0,1 & 1262 & HTRA1 & Q92743 & I \\
\hline 1216 & HNRNPK & P61978 & $c, r, s, o, I$ & 1263 & HTRA2 & 043464 & I \\
\hline 1217 & HNRNPL & P14866 & 1 & 1264 & HTT & P42858 & 0 \\
\hline 1218 & HNRNPM & P52272 & $r, o, l$ & 1265 & HUWE1 & Q7Z6Z7 & I \\
\hline 1219 & HNRNPR & 043390 & 0,1 & 1266 & HYOU1 & Q9Y4L1 & $\mathrm{I}, \mathrm{O}$ \\
\hline 1220 & HNRNPU & Q00839 & 0,1 & 1267 & $\mathrm{IAH} 1$ & Q2TAA2 & 1 \\
\hline 1221 & HNRNPUL1 & Q9BUJ2 & I & 1268 & IARS & P41252 & I \\
\hline 1222 & HNRNPUL2 & Q1KMD3 & 0,1 & 1269 & IARS2 & Q9NSE4 & I \\
\hline 1223 & HOXA4 & Q00056 & 0 & 1270 & ICAM3 & P32942 & o \\
\hline 1224 & HOXB4 & P17483 & o & 1271 & IDE & P14735 & I \\
\hline 1225 & HP & P00738 & $b, s, r, f, k, o, p, l, a$ & 1272 & IDH1 & 075874 & $r, s, c, p, o, l$ \\
\hline 1226 & HP1BP3 & Q5SSJ5 & I & 1273 & $\mathrm{IDH} 2$ & P48735 & 0,1 \\
\hline 1227 & HPR & P00739 & a & 1274 & $\mathrm{IDH} 3 \mathrm{~A}$ & P50213 & 0,1 \\
\hline 1228 & HPRT1 & P00492 & I & 1275 & IDH3G & P51553 & I \\
\hline 1229 & HPX & P02790 & $u, s, r, k, c, I$ & 1276 & IDI1 & Q13907 & I \\
\hline 1230 & HRG & P04196 & $r, l$ & 1277 & IDO1 & P14902 & I \\
\hline 1231 & HRNR & Q86YZ3 & $\mathrm{a}$ & 1278 & IFI16 & Q16666 & 1,0 \\
\hline 1232 & HSBP1 & O75506 & I & 1279 & IFI35 & P80217 & I \\
\hline 1233 & HSD11B2 & P80365 & o & 1280 & IFIT1 & P09914 & I \\
\hline 1234 & HSD17B10 & Q99714 & 1,0 & 1281 & IFIT3 & 014879 & I \\
\hline 1235 & HSD17B11 & Q8NBQ5 & o & 1282 & IFIT5 & Q13325 & I \\
\hline 1236 & HSD17B12 & Q53GQ0 & 0,1 & 1283 & IFITM1 & P13164 & o \\
\hline 1237 & HSD17B13 & Q7Z5P4 & 1,0 & 1284 & IFT122 & Q9HBG6 & I \\
\hline 1238 & HSD17B4 & P51659 & 0,1 & 1285 & IFT140 & Q96RY7 & I \\
\hline 1239 & HSD17B8 & Q92506 & 0 & 1286 & IFT172 & Q9UG01 & I \\
\hline 1240 & HSDL2 & Q6YN16 & I & 1287 & IFT20 & Q8IY31 & I \\
\hline 1241 & HSP90AA1 & P07900 & $s, r, O, I$ & 1288 & IFT27 & H0Y6C7 & I \\
\hline 1242 & HSP90AB1 & P08238 & $\mathrm{s}, \mathrm{O}, \mathrm{I}$ & 1289 & IFT46 & Q9NQC8 & I \\
\hline 1243 & HSP90AB2P & Q58FF8 & I & 1290 & IFT57 & Q9NWB7 & I \\
\hline 1244 & HSP90B1 & P14625 & $t, o, d, t, l$ & 1291 & IFT74 & Q96LB3 & $\mathrm{l}, \mathrm{h}$ \\
\hline 1245 & HSPA14 & Q0VDF9 & $\mathrm{I}, \mathrm{I}$ & 1292 & IFT80 & Q9P2H3 & I \\
\hline 1246 & HSPA1A & P0DMV8 & $\mathrm{s}, \mathrm{r}, \mathrm{c}, \mathrm{l}, \mathrm{o}, \mathrm{m}$ & 1293 & IGF1R & P08069 & o \\
\hline 1247 & HSPA1L & P34931 & 0 & 1294 & IGFALS & P35858 & I \\
\hline 1248 & HSPA2 & P54652 & 0,1 & 1295 & IGHA1 & P01876 & $q, s, k, u, r, b, l, p, q, a$ \\
\hline 1249 & HSPA4 & P34932 & o & 1296 & IGHA2 & P01877 & $\mathrm{i}, \mathrm{s}, \mathrm{k}, \mathrm{b}, \mathrm{l}, \mathrm{o}, \mathrm{a}$ \\
\hline 1250 & HSPA4L & O95757 & I & 1297 & IGHD & P01880 & I \\
\hline 1251 & HSPA5 & P11021 & $c, l, o, m, d$ & 1298 & IGHGI & P01857 & $b, u, s, k, r, l, o, a$ \\
\hline 1252 & HSPA8 & P11142 & $s, c, o, I, p, m$ & 1299 & IGHG2 & P01859 & $\mathrm{s}, \mathrm{k}, \mathrm{b}, \mathrm{l}, \mathrm{a}$ \\
\hline 1253 & HSPA9 & P38646 & $c, m, o, l, g$ & 1300 & IGHG3 & P01860 & $s, b, l, a$ \\
\hline 1254 & HSPB1 & P04792 & $\mathrm{s}, \mathrm{r}, \mathrm{k}, \mathrm{c}, \mathrm{o}, \mathrm{l}, \mathrm{p}, \mathrm{m}, \mathrm{a}$ & 1301 & IGHG4 & P01861 & $\mathrm{s}, \mathrm{l}, \mathrm{a}$ \\
\hline 1255 & HSPB11 & Q9Y547 & I & 1302 & IGHM & A0A1B0GUU9 & $b, r, s, k, b, l, p, o, a$ \\
\hline 1256 & HSPBP1 & Q9NZL4 & I & 1303 & IGHV1-2 & P23083 & I \\
\hline 1257 & HSPD1 & P10809 & $\mathrm{c}, \mathrm{l}, \mathrm{o}, \mathrm{m}, \mathrm{g}$ & 1304 & IGHV1-46 & P01743 & k \\
\hline 1258 & HSPE1 & P61604 & $\mathrm{I}, \mathrm{O}$ & 1305 & IGHV3-13 & P01766 & $r, s, l$ \\
\hline 1259 & HSPG2 & P98160 & $\mathrm{s}$ & 1306 & IGHV3-23 & P01764 & $r, k, s, l, a$ \\
\hline 1260 & HSPH1 & Q92598 & I & 1307 & IGHV3-30 & P01768 & $\mathrm{s}, \mathrm{h}$ \\
\hline 1261 & HTATIP2 & Q9BUP3 & o & 1308 & IGHV3-33 & P01772 & $k, h$ \\
\hline
\end{tabular}




\begin{tabular}{|c|c|c|c|c|c|c|c|}
\hline & Gene & $\begin{array}{l}\text { UniProt Acces- } \\
\text { sion Number }\end{array}$ & Studies & & Gene & $\begin{array}{l}\text { UniProt Acces- } \\
\text { sion Number }\end{array}$ & Studies \\
\hline 1309 & IGHV3-48 & P01763 & 1 & 1356 & ISOC1 & Q96CN7 & 1 \\
\hline 1310 & IGHV3-53 & P01767 & $\mathrm{s}, \mathrm{l}$ & 1357 & ISOC2 & Q96AB3 & 1 \\
\hline 1311 & IGHV3-7 & P01780 & $r, s, l$ & 1358 & IST1 & P53990 & I \\
\hline 1312 & IGHV4-34 & P06331 & $\mathrm{s}, \mathrm{l}$ & 1359 & ISYNA1 & Q9NPH2 & I \\
\hline 1313 & IGKC & P01834 & $\mathrm{u}, \mathrm{s}, \mathrm{k}, \mathrm{r}, \mathrm{l}, \mathrm{o}, \mathrm{a}, \mathrm{d}$ & 1360 & ITGA2 & P17301 & 0,1 \\
\hline 1314 & IGKV1-16 & P04430 & I & 1361 & ITGA3 & P26006 & o \\
\hline 1315 & IGKV1-17 & P01599 & I & 1362 & ITGA6 & P23229 & o \\
\hline 1316 & IGKV1-39 & P01597 & s & 1363 & ITGAM & P11215 & o \\
\hline 1317 & IGKV1-5 & P01602 & $\mathrm{q}, \mathrm{s}, \mathrm{l}, \mathrm{q}$ & 1364 & ITGAV & P06756 & o \\
\hline 1318 & IGKV1D-12 & P01611 & s & 1365 & ITGB1 & P05556 & 0,1 \\
\hline 1319 & IGKV1D-33 & P01593 & $s, r, b, l$ & 1366 & ITGB2 & P05107 & o \\
\hline 1320 & IGKV2D-28 & P01615 & $\mathrm{s}, \mathrm{l}$ & 1367 & ITGB4 & P16144 & o \\
\hline 1321 & IGKV3-11 & P04433 & $s, I$ & 1368 & $\mathrm{ITIH} 1$ & P19827 & 1 \\
\hline 1322 & IGKV3-20 & P01619 & $s, k, r, b, l$ & 1369 & $\mathrm{ITIH} 2$ & P19823 & I \\
\hline 1323 & IGKV4-1 & P06312 & $s, b, l$ & 1370 & $\mathrm{ITIH} 4$ & Q14624 & 1 \\
\hline 1324 & IGLC1 & POCG04 & $k, a$ & 1371 & ITLN1 & Q8WWA0 & I \\
\hline 1325 & IGLC2 & P0DOY2 & $\mathrm{b}, \mathrm{s}, \mathrm{r}, \mathrm{l}, \mathrm{o}$ & 1372 & ITPA & Q9BY32 & I \\
\hline 1326 & IGLC3 & P0DOY3 & $\mathrm{s}, \mathrm{h}$ & 1373 & ITPR1 & Q14643 & o \\
\hline 1327 & IGLC7 & A0M8Q6 & s & 1374 & ITPR2 & Q14571 & s \\
\hline 1328 & IGLL1 & P15814 & I & 1375 & ITPR3 & Q14573 & o \\
\hline 1329 & IGLL5 & B9A064 & $\mathrm{s}, \mathrm{l}$ & 1376 & IVD & P26440 & I \\
\hline 1330 & IGLV1-47 & P01700 & $s, k$ & 1377 & IVL & P07476 & $0, a$ \\
\hline 1331 & IGLV1-51 & P01701 & I & 1378 & JCHAIN & P01591 & $\mathrm{n}, \mathrm{f}, \mathrm{r}, \mathrm{b}, \mathrm{c}, \mathrm{s}, \mathrm{k}, \mathrm{q}, \mathrm{l}, \mathrm{a}, \mathrm{q}$ \\
\hline 1332 & IGLV2-11 & P01706 & $\mathrm{b}$ & 1379 & JMJD7 & POC870 & I \\
\hline 1333 & IGLV3-19 & P01714 & $s, r, l$ & 1380 & JPT1 & Q9UK76 & I \\
\hline 1334 & IGLV3-21 & P80748 & $s, k, r, l$ & 1381 & JPT2 & Q9H910 & I \\
\hline 1335 & IGLV3-25 & P01717 & $s, \mathrm{l}$ & 1382 & JUP & P14923 & $\mathrm{o}, \mathrm{l}, \mathrm{a}$ \\
\hline 1336 & IL1RN & P18510 & I & 1383 & KARS & Q15046 & I \\
\hline 1337 & ILF2 & Q12905 & 0,1 & 1384 & KCNIP3 & Q9Y2W7 & $\mathrm{h}$ \\
\hline 1338 & ILF3 & Q12906 & 0,1 & 1385 & KCNJ5 & P48544 & $\mathrm{h}$ \\
\hline 1339 & ILVBL & A1L0T0 & 1 & 1386 & KCTD12 & Q96CX2 & I \\
\hline 1340 & IMMT & Q16891 & o & 1387 & KDM5A & P29375 & s \\
\hline 1341 & IMPA1 & P29218 & I & 1388 & KHDRBS1 & Q07666 & I \\
\hline 1342 & IMPDH2 & P12268 & I & 1389 & KHSRP & Q92945 & $\mathrm{l}, \mathrm{O}$ \\
\hline 1343 & INPP1 & P49441 & I & 1390 & KIAA1217 & Q5T5P2 & o \\
\hline 1344 & INPP4B & 015327 & I & 1391 & KIAA1328 & Q86T90 & s \\
\hline 1345 & IPO4 & Q8TEX9 & I & 1392 & KIF13B & Q9NQT8 & I \\
\hline 1346 & IPO5 & 000410 & I & 1393 & KIF21A & Q7Z4S6 & 1 \\
\hline 1347 & IPO7 & 095373 & I & 1394 & $\mathrm{KIF} 2 \mathrm{~A}$ & 000139 & 1 \\
\hline 1348 & IQGAP1 & P46940 & $\mathrm{o}, \mathrm{l}, \mathrm{d}$ & 1395 & KIF5B & P33176 & 0,1 \\
\hline 1349 & IQGAP2 & Q13576 & I & 1396 & KLC4 & Q9NSK0 & 1 \\
\hline 1350 & IRF2BP2 & Q7Z5L9 & 1 & 1397 & KLHL34 & Q8N239 & s \\
\hline 1351 & IRF6 & 014896 & I & 1398 & KLK11 & Q9UBX7 & 1 \\
\hline 1352 & IRGQ & Q8WZA9 & I & 1399 & KLK7 & P49862 & $p$ \\
\hline 1353 & IRX4 & P78413 & s & 1400 & KLRF1 & Q9NZS2 & s \\
\hline 1354 & ISG15 & P05161 & 1 & 1401 & КMT2B & Q9UMN6 & o \\
\hline 1355 & ISG20 & Q96AZ6 & I & 1402 & KNG1 & P01042 & $\mathrm{r}, \mathrm{l}$ \\
\hline
\end{tabular}




\begin{tabular}{|c|c|c|c|c|c|c|c|}
\hline & Gene & $\begin{array}{l}\text { UniProt Acces- } \\
\text { sion Number }\end{array}$ & Studies & & Gene & $\begin{array}{l}\text { UniProt Acces- } \\
\text { sion Number }\end{array}$ & Studies \\
\hline 1403 & KPNA3 & 000505 & I & 1450 & KRTAP2-4 & Q9BYR9 & $\mathrm{a}$ \\
\hline 1404 & KPNA4 & 000629 & I & 1451 & KRTAP4-9 & Q9BYQ8 & $\mathrm{a}$ \\
\hline 1405 & KPNB1 & Q14974 & $\mathrm{I}, \mathrm{O}$ & 1452 & KRTAP9-8 & Q9BYQ0 & $\mathrm{a}$ \\
\hline 1406 & KPRP & Q5T749 & a & 1453 & KTN1 & Q86UP2 & 0,1 \\
\hline 1407 & KRT1 & P04264 & $b, s, r, o$ & 1454 & KYAT3 & Q6YP21 & I \\
\hline 1408 & KRT10 & P13645 & $b, s, r, o, a$ & 1455 & KYNU & Q16719 & I \\
\hline 1409 & KRT12 & Q99456 & $b$ & 1456 & LACRT & Q9GZZ8 & $\mathrm{k}, \mathrm{s}, \mathrm{r}, \mathrm{l}, \mathrm{a}$ \\
\hline 1410 & KRT13 & P13646 & $\mathrm{s}, \mathrm{O}$ & 1457 & LACTB2 & Q53H82 & I \\
\hline 1411 & KRT14 & P02533 & $b, s, o, a$ & 1458 & LAMB4 & A4D0S4 & I \\
\hline 1412 & KRT15 & P19012 & 0 & 1459 & LAMP1 & P11279 & 0,1 \\
\hline 1413 & KRT16 & P08779 & $\mathrm{b}, \mathrm{s}, \mathrm{o}, \mathrm{a}$ & 1460 & LAMTOR1 & Q6IAA8 & 0 \\
\hline 1414 & KRT17 & Q04695 & $\mathrm{o}, \mathrm{a}$ & 1461 & LANCL1 & 043813 & I \\
\hline 1415 & KRT18 & P05783 & $s, r, c, o, I$ & 1462 & LANCL2 & Q9NS86 & I \\
\hline 1416 & KRT19 & P08727 & $\mathrm{b}, \mathrm{s}, \mathrm{c}, \mathrm{o}, \mathrm{m}, \mathrm{I}$ & 1463 & LAP3 & P28838 & $c, r, l, o$ \\
\hline 1417 & KRT2 & P35908 & $s, b, o, a$ & 1464 & LARS & Q9P2J5 & 1 \\
\hline 1418 & KRT20 & P35900 & a & 1465 & LAS1L & Q9Y4W2 & o \\
\hline 1419 & KRT23 & Q9C075 & o & 1466 & LASP1 & Q14847 & $\mathrm{I}, \mathrm{O}$ \\
\hline 1420 & KRT28 & Q7Z3Y7 & $b$ & 1467 & LBR & Q14739 & o \\
\hline 1421 & KRT3 & P12035 & $b, o, a$ & 1468 & LCMT1 & Q9UIC8 & $\mathrm{h}$ \\
\hline 1422 & KRT31 & Q15323 & $\mathrm{a}$ & 1469 & LCN1 & P31025 & $n, b, u, s, r, k, f, l, a$ \\
\hline 1423 & KRT32 & Q14532 & $\mathrm{a}$ & 1470 & LCN15 & Q6UWW0 & $s, r, k, n, l$ \\
\hline 1424 & KRT33A & 076009 & a & 1471 & LCN2 & P80188 & $\mathrm{u}, \mathrm{r}, \mathrm{s}, \mathrm{b}, \mathrm{k}, \mathrm{o}, \mathrm{l}$ \\
\hline 1425 & KRT33B & Q14525 & a & 1472 & LCP1 & P13796 & $s, r, o, l$ \\
\hline 1426 & KRT34 & O76011 & a & 1473 & LCP2 & Q13094 & I \\
\hline 1427 & KRT35 & Q92764 & $\mathrm{a}$ & 1474 & LDHA & P00338 & $\mathrm{s}, \mathrm{l}, \mathrm{o}, \mathrm{a}$ \\
\hline 1428 & KRT36 & 076013 & $r, a$ & 1475 & LDHB & P07195 & $\mathrm{s}, \mathrm{r}, \mathrm{l}, \mathrm{O}$ \\
\hline 1429 & KRT37 & O76014 & $\mathrm{a}$ & 1476 & LEG1 & Q6P5S2 & $q, s, r, k, l, q$ \\
\hline 1430 & KRT38 & O76015 & $a$ & 1477 & LEMD3 & Q9Y2U8 & o \\
\hline 1431 & KRT4 & P19013 & $b, s, o, a$ & 1478 & LETM1 & 095202 & o \\
\hline 1432 & KRT5 & P13647 & $\mathrm{b}, \mathrm{s}, \mathrm{o}, \mathrm{m}, \mathrm{a}$ & 1479 & LGALS1 & P09382 & 1 \\
\hline 1433 & KRT6A & P02538 & $\mathrm{b}, \mathrm{s}, \mathrm{c}, \mathrm{O}, \mathrm{a}$ & 1480 & LGALS3 & P17931 & $\mathrm{s}, \mathrm{O}, \mathrm{I}$ \\
\hline 1434 & KRT6B & P04259 & $b, s, a$ & 1481 & LGALS3BP & Q08380 & $\mathrm{r}, \mathrm{s}, \mathrm{k}, \mathrm{l}, \mathrm{o}$ \\
\hline 1435 & KRT6C & P48668 & $b, k$ & 1482 & LGALS7 & P47929 & $\mathrm{l}, \mathrm{a}$ \\
\hline 1436 & KRT7 & P08729 & $\mathrm{s}, \mathrm{b}, \mathrm{c}, \mathrm{o}, \mathrm{a}$ & 1483 & LGALS7B & P47929 & $\mathrm{l}, \mathrm{a}$ \\
\hline 1437 & KRT76 & Q01546 & a & 1484 & LGMN & Q99538 & I \\
\hline 1438 & KRT77 & Q7Z794 & a & 1485 & LHPP & Q9H008 & I \\
\hline 1439 & KRT79 & Q5XKE5 & $\mathrm{b}, \mathrm{a}$ & 1486 & LIMA1 & Q9UHB6 & 0,1 \\
\hline 1440 & KRT8 & P05787 & $b, s, c, j, o, t, m, j, t$ & 1487 & LIMK2 & P53671 & 1 \\
\hline 1441 & KRT81 & Q14533 & a & 1488 & LLGL2 & Q6P1M3 & 0,1 \\
\hline 1442 & KRT82 & Q9NSB4 & $a$ & 1489 & LMAN1 & P49257 & 0,1 \\
\hline 1443 & KRT83 & P78385 & $\mathrm{a}$ & 1490 & LMAN2 & Q12907 & o,l \\
\hline 1444 & KRT84 & Q9NSB2 & $a$ & 1491 & LMNA & P02545 & $\mathrm{r}, \mathrm{o}, \mathrm{l}, \mathrm{a}$ \\
\hline 1445 & KRT85 & P78386 & a & 1492 & LMNB1 & P20700 & 1,0 \\
\hline 1446 & KRT86 & O43790 & a & 1493 & LMNB2 & Q03252 & 1,0 \\
\hline 1447 & KRT9 & P35527 & $b, s, r, o, a$ & 1494 & LMO7 & Q8WWI1 & 0,1 \\
\hline 1448 & KRTAP10-3 & P60369 & $\mathrm{a}$ & 1495 & LNPK & Q9C0E8 & o \\
\hline 1449 & KRTAP13-2 & Q52LG2 & a & 1496 & LONP1 & P36776 & 1 \\
\hline
\end{tabular}




\begin{tabular}{|c|c|c|c|c|c|c|c|}
\hline & Gene & $\begin{array}{l}\text { UniProt Acces- } \\
\text { sion Number }\end{array}$ & Studies & & Gene & $\begin{array}{l}\text { UniProt Acces- } \\
\text { sion Number }\end{array}$ & Studies \\
\hline 1497 & LPCAT2 & Q7L5N7 & o & 1544 & MAPK3 & P27361 & 1 \\
\hline 1498 & LPCAT3 & Q6P1A2 & o & 1545 & MAPRE1 & Q15691 & 1 \\
\hline 1499 & LPCAT4 & Q643R3 & o & 1546 & MAPRE3 & Q9UPY8 & I \\
\hline 1500 & LPGAT1 & Q92604 & o & 1547 & Mar-05 & Q9NX47 & o \\
\hline 1501 & LPO & P22079 & $\mathrm{s}, \mathrm{r}, \mathrm{l}$ & 1548 & MARCKS & P29966 & 0,1 \\
\hline 1502 & LPP & Q93052 & 1 & 1549 & MARCKSL1 & P49006 & o \\
\hline 1503 & LRBA & P50851 & 0,1 & 1550 & MARS & P56192 & $\mathrm{I}, \mathrm{O}$ \\
\hline 1504 & LRG1 & P02750 & $k, l$ & 1551 & MAT2A & P31153 & 1 \\
\hline 1505 & LRIG1 & Q96JA1 & o & 1552 & MAT2B & Q9NZL9 & 1 \\
\hline 1506 & LRP1B & Q9NZR2 & s & 1553 & MATK & P42679 & s \\
\hline 1507 & LRPPRC & P42704 & 0,1 & 1554 & MATR3 & P43243 & $\mathrm{I}, \mathrm{I}, \mathrm{O}, \mathrm{O}$ \\
\hline 1508 & LRRC46 & Q96FV0 & I & 1555 & MAX & Q6V3B1 & $\mathrm{m}$ \\
\hline 1509 & LRRC47 & Q8N1G4 & $\mathrm{l}, \mathrm{O}$ & 1556 & MB & P02144 & $\mathrm{l}, \mathrm{a}$ \\
\hline 1510 & LRRC59 & Q96AG4 & 0,1 & 1557 & MBOAT2 & Q6ZWT7 & o \\
\hline 1511 & LRRC71 & Q8N4P6 & I & 1558 & MCCC1 & Q96RQ3 & $\mathrm{l}, \mathrm{O}$ \\
\hline 1512 & LRRFIP1 & Q32MZ4 & 0,1 & 1559 & MCCC2 & Q9HCCO & I \\
\hline 1513 & LSM2 & Q9Y333 & I & 1560 & MCEMP1 & Q8IX19 & o \\
\hline 1514 & LSM3 & P62310 & I & 1561 & MCM2 & P49736 & I \\
\hline 1515 & LSM5 & Q9Y4Y9 & 1 & 1562 & MCTS1 & Q9ULC4 & I \\
\hline 1516 & LSP1 & P33241 & $\mathrm{s}, \mathrm{I}$ & 1563 & MCU & Q8NE86 & 1 \\
\hline 1517 & LSR & Q86X29 & o & 1564 & $\mathrm{MDH} 1$ & P40925 & $\mathrm{r}, \mathrm{s}, \mathrm{o}, \mathrm{l}$ \\
\hline 1518 & LSS & P48449 & o & 1565 & $\mathrm{MDH} 2$ & P40926 & $r, s, O$ \\
\hline 1519 & LTA4H & P09960 & $\mathrm{r}, \mathrm{l}, \mathrm{o}$ & 1566 & ME1 & P48163 & I \\
\hline 1520 & LTF & P02788 & $f, b, c, u, s, r, k, q, o, p, l, a, q$ & 1567 & ME2 & P23368 & 0,1 \\
\hline 1521 & LUM & P51884 & I & 1568 & METAP1 & P53582 & I \\
\hline 1522 & LXN & Q9BS40 & I & 1569 & METAP2 & P50579 & I \\
\hline 1523 & LY6D & Q14210 & o & 1570 & METTL7A & Q9H8H3 & 0,1 \\
\hline 1524 & LYN & P07948 & 0,1 & 1571 & MFN2 & 095140 & o \\
\hline 1525 & LYPLA1 & 075608 & I & 1572 & MGA & Q8IWI9 & s \\
\hline 1526 & LYPLAL1 & Q5VWZ2 & I & 1573 & MGAT1 & P26572 & o \\
\hline 1527 & LYST & Q99698 & o & 1574 & MGAT2 & Q10469 & o \\
\hline 1528 & LYZ & P61626 & $f, c, b, u, s, r, k, q, o, l, a, q$ & 1575 & MGMT & P16455 & 1 \\
\hline 1529 & LZIC & Q8WZA0 & I & 1576 & MGST1 & P10620 & 0,1 \\
\hline 1530 & LZTFL1 & Q9NQ48 & I & 1577 & MGST2 & Q99735 & o \\
\hline 1531 & M6PR & P20645 & o & 1578 & MGST3 & 014880 & 0,1 \\
\hline 1532 & MAGED2 & Q9UNF1 & I & 1579 & MICU2 & Q8IYU8 & o \\
\hline 1533 & MAGI1 & Q96QZ7 & I & 1580 & MIEN1 & Q9BRT3 & I \\
\hline 1534 & MAGOHB & Q96A72 & I & 1581 & MIER1 & Q8N108 & $\mathrm{h}$ \\
\hline 1535 & MAN2A1 & Q16706 & o & 1582 & MIF & P14174 & $s, I$ \\
\hline 1536 & MANF & P55145 & 1 & 1583 & MLEC & Q14165 & $\mathrm{I}, \mathrm{O}$ \\
\hline 1537 & MAOA & P21397 & $\mathrm{I}, \mathrm{O}$ & 1584 & MLF1 & P58340 & 1 \\
\hline 1538 & MAOB & P27338 & $\mathrm{l}, \mathrm{O}$ & 1585 & MMP10 & P09238 & 1 \\
\hline 1539 & MAP1A & P78559 & I & 1586 & MMP8 & P22894 & $s, r, l$ \\
\hline 1540 & MAP4 & P27816 & 0,1 & 1587 & MMP9 & P14780 & $s, r, o, l$ \\
\hline 1541 & MAPK1 & P28482 & I & 1588 & MMS19 & Q96T76 & I \\
\hline 1542 & MAPK11 & Q15759 & $\mathrm{h}$ & 1589 & MMUT & P22033 & 1 \\
\hline 1543 & MAPK14 & Q16539 & I & 1590 & MNAT1 & P51948 & $\mathrm{h}$ \\
\hline
\end{tabular}




\begin{tabular}{|c|c|c|c|c|c|c|c|}
\hline & Gene & $\begin{array}{l}\text { UniProt Acces- } \\
\text { sion Number }\end{array}$ & Studies & & Gene & $\begin{array}{l}\text { UniProt Acces- } \\
\text { sion Number }\end{array}$ & Studies \\
\hline 1591 & MNDA & P41218 & $\mathrm{s}, \mathrm{r}, \mathrm{O}, \mathrm{I}$ & 1638 & MYDGF & Q969H8 & 0,1 \\
\hline 1592 & MOB1B & Q7L9L4 & I & 1639 & MYF5 & P13349 & $\mathrm{h}$ \\
\hline 1593 & MOGS & Q13724 & 0,1 & 1640 & MYH10 & P35580 & 0 \\
\hline 1594 & MOS & P00540 & s & 1641 & MYH11 & P35749 & 0 \\
\hline 1595 & MPC2 & 095563 & 0 & 1642 & MYH13 & Q9UKX3 & $\mathrm{a}$ \\
\hline 1596 & MPI & P34949 & I & 1643 & MYH14 & Q7Z406 & 0,1 \\
\hline 1597 & MPO & P05164 & $s, r, k, o, I$ & 1644 & MYH9 & P35579 & $\mathrm{s}, \mathrm{r}, \mathrm{O}, \mathrm{l}, \mathrm{a}$ \\
\hline 1598 & MPST & P25325 & I & 1645 & MYL12A & P19105 & I \\
\hline 1599 & MRI1 & Q9BV20 & I & 1646 & MYL4 & P12829 & $\mathrm{j}, \mathrm{j}$ \\
\hline 1600 & MRPL12 & P52815 & I & 1647 & MYL6 & P60660 & $\mathrm{s}, \mathrm{O}, \mathrm{l}$ \\
\hline 1601 & MRPL15 & Q9P015 & 0 & 1648 & MYO1B & 043795 & 0,1 \\
\hline 1602 & MRPS31 & Q92665 & I & 1649 & MYO1C & O00159 & 0 \\
\hline 1603 & $\mathrm{MSH} 2$ & P43246 & I & 1650 & MYO1D & O94832 & $\mathrm{I}, \mathrm{O}$ \\
\hline 1604 & MSI2 & Q96DH6 & I & 1651 & MYO1E & Q12965 & 1 \\
\hline 1605 & MSLN & Q13421 & $s, k, l$ & 1652 & MYO1G & B0I1T2 & $\mathrm{m}$ \\
\hline 1606 & MSMB & P08118 & $s, r, k, l$ & 1653 & MYO5B & Q9ULV0 & $\mathrm{I}, \mathrm{O}$ \\
\hline 1607 & MSN & P26038 & $\mathrm{s}, \mathrm{O}, \mathrm{l}$ & 1654 & MYO5C & Q9NQX4 & o \\
\hline 1608 & MSRA & Q9UJ68 & I & 1655 & MYO6 & Q9UM54 & 0,1 \\
\hline 1609 & MST1R & Q04912 & 0 & 1656 & MYOF & Q9NZM1 & 0,1 \\
\hline 1610 & MT-CO2 & P00403 & 0,1 & 1657 & MYOZ2 & Q9NPC6 & s \\
\hline 1611 & MT-ND1 & P03886 & 0 & 1658 & NAA15 & Q9BXJ9 & I \\
\hline 1612 & MT-ND4 & P03905 & 0 & 1659 & NAA38 & Q9BRA0 & I \\
\hline 1613 & MT-ND5 & P03915 & 0,1 & 1660 & NAA50 & Q9GZZ1 & I \\
\hline 1614 & MTAP & Q13126 & I & 1661 & NACA & Q13765 & 0,1 \\
\hline 1615 & MTCH1 & Q9NZJ7 & 0,1 & 1662 & NAE1 & Q13564 & I \\
\hline 1616 & MTCH2 & Q9Y6C9 & 0,1 & 1663 & NAGK & Q9UJ70 & 1,0 \\
\hline 1617 & MTDH & Q86UE4 & o & 1664 & NAGLU & P54802 & I \\
\hline 1618 & MTFP1 & Q9UDX5 & o & 1665 & NAMPT & P43490 & $\mathrm{r}, \mathrm{l}, \mathrm{o}$ \\
\hline 1619 & MTHFD1 & P11586 & I & 1666 & NANS & Q9NR45 & 0,1 \\
\hline 1620 & MTPN & P58546 & I & 1667 & NAP1L1 & P55209 & I \\
\hline 1621 & MTREX & P42285 & I & 1668 & NAP1L4 & Q99733 & 0,1 \\
\hline 1622 & MTX1 & Q13505 & 0 & 1669 & NAPA & P54920 & I \\
\hline 1623 & MUC1 & P15941 & 0,1 & 1670 & NAPG & Q99747 & 1 \\
\hline 1624 & MUC13 & Q9H3R2 & 0 & 1671 & NAPRT & Q6XQN6 & I \\
\hline 1625 & MUC16 & Q8WXI7 & I & 1672 & NARS & 043776 & I \\
\hline 1626 & MUC2 & Q02817 & 0,1 & 1673 & NASP & P49321 & 0,1 \\
\hline 1627 & MUC4 & Q99102 & 0,1 & 1674 & NAT1 & P18440 & I \\
\hline 1628 & MUC5AC & P98088 & $\mathrm{b}, \mathrm{s}, \mathrm{k}, \mathrm{I}$ & 1675 & NAT10 & Q9H0A0 & 0 \\
\hline 1629 & MUC5B & Q9HC84 & $b, q, s, r, k, l, q$ & 1676 & NAXD & Q8IW45 & 1 \\
\hline 1630 & MUC7 & Q8TAX7 & $s, r, k$ & 1677 & NAXE & Q8NCW5 & I \\
\hline 1631 & MVK & Q03426 & I & 1678 & NCBP1 & Q09161 & I \\
\hline 1632 & MVP & Q14764 & 0,1 & 1679 & NCEH1 & Q6PIU2 & o \\
\hline 1633 & MX1 & P20591 & 1,0 & 1680 & NCF1C & A8MVU1 & I \\
\hline 1634 & MXRA7 & P84157 & o & 1681 & NCF4 & Q15080 & $\mathrm{h}$ \\
\hline 1635 & MYADM & Q96S97 & o & 1682 & NCKAP1 & Q9Y2A7 & 0,1 \\
\hline 1636 & MYBBP1A & Q9BQG0 & 0 & 1683 & $\mathrm{NCL}$ & P19338 & 0,1 \\
\hline 1637 & MYCBP & Q99417 & I & 1684 & NCLN & Q969V3 & 0 \\
\hline
\end{tabular}




\begin{tabular}{|c|c|c|c|c|c|c|c|}
\hline & Gene & $\begin{array}{l}\text { UniProt Acces- } \\
\text { sion Number }\end{array}$ & Studies & & Gene & $\begin{array}{l}\text { UniProt Acces- } \\
\text { sion Number }\end{array}$ & Studies \\
\hline 1685 & NCS1 & P62166 & $\mathrm{h}$ & 1732 & NME4 & 000746 & j, j \\
\hline 1686 & NCSTN & Q92542 & o & 1733 & NME5 & P56597 & 1 \\
\hline 1687 & NDC1 & Q9BTX1 & o & 1734 & NMES1 & Q9C002 & I \\
\hline 1688 & NDE1 & Q9NXR1 & $\mathrm{h}$ & 1735 & NMI & Q13287 & I \\
\hline 1689 & NDRG2 & Q9UN36 & I & 1736 & NMRAL1 & Q9HBL8 & I \\
\hline 1690 & NDUFA10 & 095299 & 1 & 1737 & NMT1 & P30419 & 1 \\
\hline 1691 & NDUFA11 & Q86Y39 & I & 1738 & NNT & Q13423 & $\mathrm{I}, \mathrm{O}$ \\
\hline 1692 & NDUFA12 & Q9U109 & o & 1739 & NOL3 & 060936 & 1 \\
\hline 1693 & NDUFA13 & Q9P0J0 & 0,1 & 1740 & NOLC1 & Q14978 & $\mathrm{l}, \mathrm{O}$ \\
\hline 1694 & NDUFA4 & 000483 & 0 & 1741 & NONO & Q15233 & 0,1 \\
\hline 1695 & NDUFA4L2 & Q9NRX3 & I & 1742 & NOP56 & 000567 & o \\
\hline 1696 & NDUFA5 & Q16718 & I & 1743 & NOP58 & Q9Y2X3 & $\mathrm{I}, \mathrm{O}$ \\
\hline 1697 & NDUFA9 & Q16795 & $\mathrm{l}, \mathrm{O}$ & 1744 & NOS2 & P35228 & o \\
\hline 1698 & NDUFB10 & 096000 & o & 1745 & NPC1 & 015118 & 0 \\
\hline 1699 & NDUFB4 & 095168 & 0,1 & 1746 & NPC2 & P61916 & $s, r, I$ \\
\hline 1700 & NDUFB6 & 095139 & o & 1747 & NPEPL1 & Q8NDH3 & I \\
\hline 1701 & NDUFB7 & P17568 & o & 1748 & NPEPPS & P55786 & 0,1 \\
\hline 1702 & NDUFB8 & O95169 & o & 1749 & NPLOC4 & Q8TAT6 & I \\
\hline 1703 & NDUFC2 & O95298 & o & 1750 & NPM1 & P06748 & $\mathrm{l}, \mathrm{O}$ \\
\hline 1704 & NDUFS1 & P28331 & 0,1 & 1751 & NPR3 & P17342 & $\mathrm{h}$ \\
\hline 1705 & NDUFS2 & 075306 & $\mathrm{l}, \mathrm{O}$ & 1752 & NPTN & Q9Y639 & $\mathrm{l}, \mathrm{O}$ \\
\hline 1706 & NDUFS3 & O75489 & $\mathrm{l}, \mathrm{m}, \mathrm{O}$ & 1753 & NQO1 & P15559 & $\mathrm{s}, \mathrm{r}, \mathrm{l}, \mathrm{O}$ \\
\hline 1707 & NDUFS5 & O43920 & 1 & 1754 & NQO2 & P16083 & I \\
\hline 1708 & NDUFS7 & 075251 & o & 1755 & NRBP1 & Q9UHY1 & I \\
\hline 1709 & NDUFS8 & 000217 & $0, j, j$ & 1756 & NRDC & O43847 & 1 \\
\hline 1710 & NDUFV1 & P49821 & I & 1757 & NSDHL & Q15738 & o \\
\hline 1711 & NDUFV2 & P19404 & I & 1758 & NSF & P46459 & 0,1 \\
\hline 1712 & NEB & P20929 & o & 1759 & NSFL1C & Q9UNZ2 & I \\
\hline 1713 & NECAP2 & Q9NVZ3 & I & 1760 & NSUN2 & Q08J23 & 1 \\
\hline 1714 & NEDD8 & Q15843 & I & 1761 & NT5C & Q8TCD5 & I \\
\hline 1715 & NEFL & P07196 & $\mathrm{h}$ & 1762 & NT5C2 & P49902 & I \\
\hline 1716 & NEO1 & Q92859 & s & 1763 & NT5C3A & Q9H0P0 & 1 \\
\hline 1717 & NFKB1 & P19838 & I & 1764 & NT5DC1 & Q5TFE4 & 1 \\
\hline 1718 & NHLRC2 & Q8NBF2 & I & 1765 & NUCB1 & Q02818 & 0,1 \\
\hline 1719 & NIBAN1 & Q9BZQ8 & 0,1 & 1766 & NUCB2 & P80303 & $\mathrm{s}, \mathrm{r}, \mathrm{k}, \mathrm{o}, \mathrm{l}$ \\
\hline 1720 & NIBAN2 & Q96TA1 & 0,1 & 1767 & NUCKS1 & Q9H1E3 & I \\
\hline 1721 & NIF3L1 & Q9GZT8 & I & 1768 & NUDC & Q9Y266 & 0,1 \\
\hline 1722 & NIPSNAP1 & Q9BPW8 & o & 1769 & NUDCD2 & Q8WVJ2 & 1 \\
\hline 1723 & NIPSNAP2 & 075323 & 0,1 & 1770 & NUDT12 & Q9BQG2 & I \\
\hline 1724 & NIPSNAP3A & Q9UFNO & I & 1771 & NUDT14 & 095848 & I \\
\hline 1725 & NIT1 & Q86X76 & I & 1772 & NUDT15 & Q9NV35 & 1 \\
\hline 1726 & NIT2 & Q9NQR4 & 1 & 1773 & NUDT16 & Q96DE0 & 1 \\
\hline 1727 & NLN & Q9BYT8 & 0 & 1774 & NUDT16L1 & Q9BRJ7 & 1 \\
\hline 1728 & NME1 & P15531 & $p$ & 1775 & NUDT2 & P50583 & I \\
\hline 1729 & NME2 & P22392 & $r, p$ & 1776 & NUDT21 & 043809 & I \\
\hline 1730 & NME2P1 & O60361 & I & 1777 & NUDT5 & Q9UKK9 & 1 \\
\hline 1731 & NME3 & Q13232 & o & 1778 & NUDT9 & Q9BW91 & I \\
\hline
\end{tabular}




\begin{tabular}{|c|c|c|c|c|c|c|c|}
\hline & Gene & $\begin{array}{l}\text { UniProt Acces- } \\
\text { sion Number }\end{array}$ & Studies & & Gene & $\begin{array}{l}\text { UniProt Acces- } \\
\text { sion Number }\end{array}$ & Studies \\
\hline 1779 & NUMA1 & Q14980 & 0,1 & 1826 & PAICS & P22234 & I \\
\hline 1780 & NUP155 & 075694 & 0 & 1827 & PAIP1 & Q9H074 & I \\
\hline 1781 & NUP160 & Q12769 & 0 & 1828 & PAK1 & Q13153 & I \\
\hline 1782 & NUP205 & Q92621 & 1,0 & 1829 & PAK2 & Q13177 & I \\
\hline 1783 & NUP210 & Q8TEM1 & 0 & 1830 & PALLD & Q8WX93 & I \\
\hline 1784 & NUP62 & P37198 & I & 1831 & PAPSS1 & 043252 & I \\
\hline 1785 & NUP93 & Q8N1F7 & $\mathrm{I}, \mathrm{O}$ & 1832 & PAPSS2 & 095340 & 1 \\
\hline 1786 & NUTF2 & P61970 & I & 1833 & PARK7 & Q99497 & $s, r, j, m, o, l, j, d$ \\
\hline 1787 & NXN & Q6DKJ4 & I & 1834 & PARP1 & P09874 & I \\
\hline 1788 & OAS2 & P29728 & $\mathrm{I}, \mathrm{O}$ & 1835 & PARP10 & Q53GL7 & I \\
\hline 1789 & OAS3 & Q9Y6K5 & $\mathrm{I}, \mathrm{O}$ & 1836 & PARP4 & Q9UKK3 & $s, 1$ \\
\hline 1790 & OAT & P04181 & 0,1 & 1837 & PARP9 & Q8IXQ6 & I \\
\hline 1791 & $\mathrm{OBP} 2 \mathrm{~A}$ & Q9NY56 & C & 1838 & PATJ & Q8NI35 & $\mathrm{s}$ \\
\hline 1792 & OBSCN & Q5VST9 & 0 & 1839 & PBDC1 & Q9BVG4 & 1 \\
\hline 1793 & OCIAD2 & Q56VL3 & I & 1840 & PBLD & P30039 & 1 \\
\hline 1794 & ODF3B & A8MYP8 & I & 1841 & PCBD1 & P61457 & I \\
\hline 1795 & $\mathrm{OGDH}$ & Q02218 & 0,1 & 1842 & PCBD2 & Q9H0N5 & I \\
\hline 1796 & OGFR & Q9NZT2 & I & 1843 & PCBP1 & Q15365 & 0,1 \\
\hline 1797 & OLA1 & Q9NTK5 & 0,1 & 1844 & PCBP2 & Q15366 & 0,1 \\
\hline 1798 & OPA 1 & 060313 & 0,1 & 1845 & PCK2 & Q16822 & I \\
\hline 1799 & OPLAH & 014841 & I & 1846 & PCM1 & Q15154 & 0,1 \\
\hline 1800 & OPRPN & Q99935 & $\mathrm{s}, \mathrm{r}, \mathrm{k}, \mathrm{l}$ & 1847 & PCMT1 & P22061 & I \\
\hline 1801 & OR1L6 & Q8NGR2 & s & 1848 & PCNA & P12004 & I \\
\hline 1802 & ORM1 & P02763 & $\mathrm{s}, \mathrm{r}, \mathrm{u}, \mathrm{q}, \mathrm{l}, \mathrm{a}, \mathrm{q}$ & 1849 & PCNT & 095613 & $\mathrm{I}, \mathrm{O}$ \\
\hline 1803 & ORM2 & P19652 & $s, I$ & 1850 & PCYOX1 & Q9UHG3 & o \\
\hline 1804 & OSBP & P22059 & I & 1851 & PCYT1B & Q9Y5K3 & I \\
\hline 1805 & OSBPL5 & Q9H0X9 & o & 1852 & PDAP1 & Q13442 & I \\
\hline 1806 & OSBPL8 & Q9BZF1 & o & 1853 & PDCD10 & Q9BUL8 & I \\
\hline 1807 & OSCP1 & Q8WVF1 & I & 1854 & PDCD11 & Q14690 & 0 \\
\hline 1808 & OSTF1 & Q92882 & I & 1855 & PDCD4 & Q53EL6 & 1 \\
\hline 1809 & OTOR & Q9NRC9 & $\mathrm{h}$ & 1856 & PDCD5 & 014737 & I \\
\hline 1810 & OTUB1 & Q96FW1 & 0,1 & 1857 & PDCD6 & 075340 & I \\
\hline 1811 & OXA1L & Q15070 & $\mathrm{I}, \mathrm{O}$ & 1858 & PDCD6IP & Q8WUM4 & 1 \\
\hline 1812 & OXCT1 & P55809 & 0,1 & 1859 & PDCL & Q13371 & $\mathrm{h}$ \\
\hline 1813 & OXR1 & Q8N573 & I & 1860 & PDE12 & F6T1Q0 & I \\
\hline 1814 & OXSR1 & 095747 & I & 1861 & PDE1A & P54750 & $\mathrm{h}$ \\
\hline 1815 & $\mathrm{P} 3 \mathrm{H} 1$ & Q32P28 & $\mathrm{h}$ & 1862 & PDE9A & 076083 & $\mathrm{~h}$ \\
\hline 1816 & $\mathrm{P} 4 \mathrm{HB}$ & P07237 & $r, m, o, l, p, d$ & 1863 & PDHA1 & P08559 & 1,0 \\
\hline 1817 & PA2G4 & Q9UQ80 & I & 1864 & PDHB & P11177 & j, j j \\
\hline 1818 & PABPC1 & P11940 & 0,1 & 1865 & PDIA3 & P30101 & $\mathrm{r}, \mathrm{c}, \mathrm{m}, \mathrm{o}, \mathrm{I}$ \\
\hline 1819 & PABPC4 & Q13310 & I & 1866 & PDIA4 & P13667 & 0,1 \\
\hline 1820 & PABPN1 & Q86U42 & I & 1867 & PDIA6 & Q15084 & 0,1 \\
\hline 1821 & PACRG & Q96M98 & I & 1868 & PDLIM1 & 000151 & 0,1 \\
\hline 1822 & PACSIN2 & Q9UNF0 & I & 1869 & PDLIM4 & P50479 & I \\
\hline 1823 & PAFAH1B1 & P43034 & 0,1 & 1870 & PDLIM5 & Q96HC4 & I \\
\hline 1824 & PAFAH1B2 & P68402 & I & 1871 & PDS5A & Q29RF7 & r \\
\hline 1825 & PAFAH1B3 & Q15102 & I & 1872 & PDXDC1 & Q6P996 & I \\
\hline
\end{tabular}




\begin{tabular}{|c|c|c|c|c|c|c|c|}
\hline & Gene & $\begin{array}{l}\text { UniProt Acces- } \\
\text { sion Number }\end{array}$ & Studies & & Gene & $\begin{array}{l}\text { UniProt Acces- } \\
\text { sion Number }\end{array}$ & Studies \\
\hline 1873 & PDXK & 000764 & 0,1 & 1920 & PITRM1 & Q5JRX3 & 1 \\
\hline 1874 & PEA15 & Q15121 & 1 & 1921 & PKM & P14618 & $\mathrm{s}, \mathrm{r}, \mathrm{c}, \mathrm{o}, \mathrm{l}, \mathrm{p}, \mathrm{a}$ \\
\hline 1875 & PEBP1 & P30086 & $\mathrm{r}, \mathrm{s}, \mathrm{p}, \mathrm{t}, \mathrm{l}, \mathrm{o,t}$ & 1922 & PKP1 & Q13835 & $0, a$ \\
\hline 1876 & PEBP4 & Q96S96 & I & 1923 & PKP2 & Q99959 & $\mathrm{l}, \mathrm{O}$ \\
\hline 1877 & PELP1 & Q8IZL8 & o & 1924 & PKP3 & Q9Y446 & o \\
\hline 1878 & PEPD & P12955 & 1 & 1925 & PLA2G2A & P14555 & $s, r, k$ \\
\hline 1879 & PEX11B & 096011 & o & 1926 & PLAA & Q9Y263 & I \\
\hline 1880 & PFAS & 015067 & 1 & 1927 & PLBD1 & Q6P4A8 & 1 \\
\hline 1881 & PFDN2 & Q9UHV9 & I & 1928 & PLCL2 & Q9UPR0 & b \\
\hline 1882 & PFDN4 & Q9NQP4 & I & 1929 & PLD2 & 014939 & o \\
\hline 1883 & PFDN5 & Q99471 & I & 1930 & PLEC & Q15149 & $\mathrm{I}, \mathrm{O}$ \\
\hline 1884 & PFDN6 & 015212 & I & 1931 & PLEK & P08567 & I \\
\hline 1885 & PFKL & P17858 & $\mathrm{l}, \mathrm{O}$ & 1932 & PLEKHJ1 & Q9NW61 & 1 \\
\hline 1886 & PFKM & P08237 & I & 1933 & PLG & P00747 & $b, l$ \\
\hline 1887 & PFKP & Q01813 & $\mathrm{I}, \mathrm{O}$ & 1934 & PLGRKT & Q9HBL7 & o \\
\hline 1888 & PFN1 & P07737 & $s, r, k, o, l$ & 1935 & PLIN3 & 060664 & I \\
\hline 1889 & PFN2 & P35080 & I & 1936 & PLLP & Q9Y342 & o \\
\hline 1890 & PGAM1 & P18669 & $c, s, r, o, l$ & 1937 & PLPBP & 094903 & I \\
\hline 1891 & PGD & P52209 & $s, r, p, h, l, o$ & 1938 & PLPP3 & 014495 & o \\
\hline 1892 & PGK1 & P00558 & $s, r, o, l$ & 1939 & PLS1 & Q14651 & 0,1 \\
\hline 1893 & PGLS & 095336 & 0,1 & 1940 & PLS3 & P13797 & 1 \\
\hline 1894 & PGLYRP1 & O75594 & $s, \mathrm{l}$ & 1941 & PLTP & P55058 & $r, l$ \\
\hline 1895 & PGLYRP2 & Q96PD5 & I & 1942 & PML & P29590 & 0,1 \\
\hline 1896 & PGM1 & P36871 & 0,1 & 1943 & PMM2 & 015305 & I \\
\hline 1897 & PGM2 & Q96G03 & I & 1944 & PMPCA & Q10713 & I \\
\hline 1898 & PGM2L1 & Q6PCE3 & I & 1945 & PMPCB & O75439 & I \\
\hline 1899 & PGM3 & 095394 & I & 1946 & PMVK & Q15126 & I \\
\hline 1900 & PGP & A6NDG6 & I & 1947 & PNN & Q9H307 & o \\
\hline 1901 & PGRMC1 & 000264 & $\mathrm{l}, \mathrm{O}$ & 1948 & PNP & P00491 & 1 \\
\hline 1902 & PGRMC2 & 015173 & 0 & 1949 & PNPO & Q9NVS9 & I \\
\hline 1903 & PHB & P35232 & $\mathrm{c}, \mathrm{O}, \mathrm{l}, \mathrm{m}$ & 1950 & POC5 & Q8NA72 & o \\
\hline 1904 & PHB2 & Q99623 & 0,1 & 1951 & PODXL & 000592 & o \\
\hline 1905 & PHF6 & Q8IWS0 & s & 1952 & POF1B & Q8WVV4 & 0,1 \\
\hline 1906 & PHGDH & O43175 & I & 1953 & POLE2 & P56282 & s \\
\hline 1907 & PHPT1 & Q9NRX4 & $\mathrm{l}, \mathrm{h}$ & 1954 & POLR1C & 015160 & I \\
\hline 1908 & PHYHD1 & Q5SRE7 & I & 1955 & POLR2B & P30876 & I \\
\hline 1909 & $\mathrm{PI} 3$ & P19957 & $r, k, l$ & 1956 & POLR2H & P52434 & I \\
\hline 1910 & PIGG & Q5H8A4 & o & 1957 & PON1 & P27169 & $r, l$ \\
\hline 1911 & PIGO & Q8TEQ8 & o & 1958 & POR & P16435 & 0,1 \\
\hline 1912 & PIGR & P01833 & $r, s, u, k, b, c, o, p, l, a$ & 1959 & POTEE & Q6S8J3 & o \\
\hline 1913 & PIN1 & Q13526 & I & 1960 & POTEJ & P0CG39 & 1 \\
\hline 1914 & PIP & P12273 & $n, f, b, s, r, k, u, l, p, a$ & 1961 & PPA1 & Q15181 & j, j \\
\hline 1915 & PIP4K2C & Q8TBX8 & I & 1962 & PPA2 & Q9H2U2 & 0,1 \\
\hline 1916 & PIR & 000625 & I & 1963 & PPCS & Q9HAB8 & I \\
\hline 1917 & PITHD1 & Q9GZP4 & I & 1964 & PPIA & P62937 & $s, r, o, p, l$ \\
\hline 1918 & PITPNA & Q00169 & I & 1965 & PPIB & P23284 & 0,1 \\
\hline 1919 & PITPNB & P48739 & I & 1966 & PPIC & P45877 & I \\
\hline
\end{tabular}




\begin{tabular}{|c|c|c|c|c|c|c|c|}
\hline & Gene & $\begin{array}{l}\text { UniProt Acces- } \\
\text { sion Number }\end{array}$ & Studies & & Gene & $\begin{array}{l}\text { UniProt Acces- } \\
\text { sion Number }\end{array}$ & Studies \\
\hline 1967 & PPID & Q08752 & I & 2014 & PRKAR2A & P13861 & $\mathrm{l}, \mathrm{o}, \mathrm{h}$ \\
\hline 1968 & PPIL1 & Q9Y3C6 & I & 2015 & PRKCD & Q05655 & I \\
\hline 1969 & PPIL3 & $\mathrm{Q} 9 \mathrm{H} 2 \mathrm{H} 8$ & 1 & 2016 & PRKCSH & P14314 & 1,0 \\
\hline 1970 & PPL & O60437 & 0,1 & 2017 & PRKDC & P78527 & 0,1 \\
\hline 1971 & PPM1B & O75688 & 1 & 2018 & PRKRA & 075569 & I \\
\hline 1972 & PPM1F & P49593 & I & 2019 & PRMT1 & Q99873 & 1 \\
\hline 1973 & PPM1G & 015355 & I & 2020 & PRMT5 & 014744 & I \\
\hline 1974 & PPME1 & Q9Y570 & I & 2021 & PRODH & 043272 & $\mathrm{o,h}$ \\
\hline 1975 & PPOX & P50336 & 0,1 & 2022 & PROM1 & 043490 & 0,1 \\
\hline 1976 & PPP1CA & P62136 & 0,1 & 2023 & PROS1 & P07225 & I \\
\hline 1977 & PPP1CB & P62140 & I & 2024 & PRPF19 & Q9UMS4 & 0,1 \\
\hline 1978 & PPP1CC & P36873 & I & 2025 & PRPF6 & 094906 & 0,1 \\
\hline 1979 & PPP1R1A & Q13522 & I & 2026 & PRPF8 & Q6P2Q9 & 0,1 \\
\hline 1980 & PPP1R7 & Q15435 & I & 2027 & PRPS1 & P60891 & I \\
\hline 1981 & PPP1R8 & Q12972 & 1 & 2028 & PRPS2 & P11908 & I \\
\hline 1982 & PPP2CA & P67775 & $h, l$ & 2029 & PRPSAP1 & Q14558 & I \\
\hline 1983 & PPP2CB & P62714 & I & 2030 & PRPSAP2 & 060256 & I \\
\hline 1984 & PPP2R1A & Р30153 & 0,1 & 2031 & PRR15 & Q8IV56 & I \\
\hline 1985 & PPP2R2A & P63151 & 1 & 2032 & PRR4 & Q16378 & $b, s, r, k, l$ \\
\hline 1986 & РPРЗСА & Q08209 & I & 2033 & PRRC1 & Q96M27 & I \\
\hline 1987 & PPP4C & P60510 & I & 2034 & PRRC2A & P48634 & 0 \\
\hline 1988 & PPP4R3B & Q5MIZ7 & I & 2035 & PRSS8 & Q16651 & 0 \\
\hline 1989 & PРP5C & P53041 & I & 2036 & PRTN3 & P24158 & $s, r, l, o$ \\
\hline 1990 & РPР6C & 000743 & I & 2037 & PRUNE2 & Q8WUY3 & I \\
\hline 1991 & PPT1 & P50897 & 1 & 2038 & PRXL2A & Q9BRX8 & 0,1 \\
\hline 1992 & PRAF2 & O60831 & o & 2039 & PSAP & P07602 & $s, r, l, o$ \\
\hline 1993 & PRAM1 & Q96QH2 & $\mathrm{s}$ & 2040 & PSEN1 & P49768 & 0 \\
\hline 1994 & PRB1 & P04280 & $\mathrm{k}$ & 2041 & PSIP1 & 075475 & I \\
\hline 1995 & PRB2 & P02812 & $c, s, r, k, a$ & 2042 & PSMA1 & P25786 & 1,0 \\
\hline 1996 & PRB3 & Q04118 & o,a & 2043 & PSMA2 & P25787 & 1 \\
\hline 1997 & PRB4 & P10163 & $\mathrm{k}, \mathrm{c}$ & 2044 & РSMA3 & P25788 & 1,0 \\
\hline 1998 & PRDM1 & 075626 & 1 & 2045 & PSMA4 & P25789 & I \\
\hline 1999 & PRDX1 & Q06830 & $c, s, r, o, m, l, p$ & 2046 & PSMA5 & P28066 & $s, l, p$ \\
\hline 2000 & PRDX2 & P32119 & $c, s, b, j, o, m, l, p, a, j$ & 2047 & PSMA6 & P60900 & I \\
\hline 2001 & PRDX3 & P30048 & 0,1 & 2048 & PSMA7 & 014818 & I \\
\hline 2002 & PRDX4 & Q13162 & 0,1 & 2049 & PSMB1 & P20618 & $r, l$ \\
\hline 2003 & PRDX5 & P30044 & $c, s, r, e, o, p, l$ & 2050 & PSMB10 & P40306 & I \\
\hline 2004 & PRDX6 & P30041 & $\mathrm{c}, \mathrm{s}, \mathrm{r}, \mathrm{O}, \mathrm{m}, \mathrm{I}$ & 2051 & PSMB2 & P49721 & $\mathrm{I}, \mathrm{O}$ \\
\hline 2005 & PREB & Q9HCU5 & 0 & 2052 & PSMB3 & P49720 & 1 \\
\hline 2006 & PREP & P48147 & 1 & 2053 & PSMB4 & P28070 & I \\
\hline 2007 & PRG4 & Q92954 & o & 2054 & PSMB6 & P28072 & 1 \\
\hline 2008 & PRH1 & P02810 & $s, a$ & 2055 & PSMB7 & Q99436 & I \\
\hline 2009 & $\mathrm{PRH} 2$ & P02810 & $s, a$ & 2056 & PSMB8 & P28062 & I \\
\hline 2010 & PRKAA1 & Q13131 & I & 2057 & PSMB9 & P28065 & I \\
\hline 2011 & PRKACA & P17612 & I & 2058 & PSMC1 & P62191 & I \\
\hline 2012 & PRKAG1 & P54619 & 1 & 2059 & PSMC2 & P35998 & $\mathrm{I}, \mathrm{O}$ \\
\hline 2013 & PRKAR1A & P10644 & 1 & 2060 & PSMC3 & P17980 & $\mathrm{I}, \mathrm{O}$ \\
\hline
\end{tabular}




\begin{tabular}{|c|c|c|c|c|c|c|c|}
\hline & Gene & $\begin{array}{l}\text { UniProt Acces- } \\
\text { sion Number }\end{array}$ & Studies & & Gene & $\begin{array}{l}\text { UniProt Acces- } \\
\text { sion Number }\end{array}$ & Studies \\
\hline 2061 & PSMC4 & P43686 & 1 & 2108 & PYCARD & Q9ULZ3 & 1 \\
\hline 2062 & PSMC5 & P62195 & 1 & 2109 & PYCR3 & Q53H96 & I \\
\hline 2063 & PSMC6 & P62333 & I & 2110 & PYGB & P11216 & $\mathrm{r}, \mathrm{l}, \mathrm{o}$ \\
\hline 2064 & PSMD1 & Q99460 & I & 2111 & PYGL & P06737 & I \\
\hline 2065 & PSMD10 & 075832 & I & 2112 & PYM1 & Q9BRP8 & 1 \\
\hline 2066 & PSMD11 & 000231 & $p, l$ & 2113 & QARS & P47897 & $\mathrm{I}, \mathrm{O}$ \\
\hline 2067 & PSMD12 & 000232 & I & 2114 & QDPR & P09417 & I \\
\hline 2068 & PSMD13 & Q9UNM6 & I & 2115 & QSOX1 & 000391 & $s, r, o, l$ \\
\hline 2069 & PSMD14 & 000487 & $\mathrm{l}, \mathrm{h}$ & 2116 & RAB10 & P61026 & $\mathrm{I}, \mathrm{O}$ \\
\hline 2070 & PSMD2 & Q13200 & $\mathrm{l}, \mathrm{O}$ & 2117 & RAB11A & P62491 & o \\
\hline 2071 & PSMD3 & O43242 & 1 & 2118 & RAB11B & Q15907 & 1 \\
\hline 2072 & PSMD4 & P55036 & 1 & 2119 & RAB13 & P51153 & 0,1 \\
\hline 2073 & PSMD5 & Q16401 & I & 2120 & RAB14 & P61106 & $\mathrm{I}, \mathrm{O}$ \\
\hline 2074 & PSMD6 & Q15008 & I & 2121 & RAB18 & Q9NP72 & I \\
\hline 2075 & PSMD7 & P51665 & I & 2122 & RAB1A & P62820 & o \\
\hline 2076 & PSMD8 & P48556 & I & 2123 & RAB1B & Q9H0U4 & $\mathrm{I}, \mathrm{O}$ \\
\hline 2077 & PSMD9 & 000233 & I & 2124 & RAB21 & Q9UL25 & 1,0 \\
\hline 2078 & PSME1 & Q06323 & $r, o, j, l, h, j$ & 2125 & RAB22A & Q9UL26 & o \\
\hline 2079 & PSME2 & Q9UL46 & $\mathrm{r}, \mathrm{o}, \mathrm{l}$ & 2126 & RAB2A & P61019 & 0,1 \\
\hline 2080 & PSME3 & P61289 & I & 2127 & RAB35 & Q15286 & o \\
\hline 2081 & PSME4 & Q14997 & I & 2128 & RAB37 & Q96AX2 & o \\
\hline 2082 & PSMF1 & Q92530 & 1 & 2129 & RAB3C & Q96E17 & $\mathrm{h}$ \\
\hline 2083 & PSMG2 & Q969U7 & I & 2130 & RAB3D & 095716 & $\mathrm{I}, \mathrm{O}$ \\
\hline 2084 & PSPC1 & Q8WXF1 & I & 2131 & RAB5A & P20339 & o \\
\hline 2085 & PTBP1 & P26599 & $\mathrm{s}, \mathrm{o}, \mathrm{l}$ & 2132 & RAB5B & P61020 & 1,0 \\
\hline 2086 & РTBP3 & O95758 & I & 2133 & RAB5C & P51148 & 0,1 \\
\hline 2087 & PTDSS2 & Q9BVG9 & o & 2134 & RAB6A & P20340 & 0,1 \\
\hline 2088 & PTER & Q96BW5 & 1 & 2135 & RAB7A & P51149 & $\mathrm{I}, \mathrm{O}$ \\
\hline 2089 & PTGES & 014684 & o & 2136 & RAB8A & P61006 & 1 \\
\hline 2090 & PTGES3 & Q15185 & I & 2137 & RABGGTA & Q92696 & $\mathrm{l}, \mathrm{h}$ \\
\hline 2091 & PTGFRN & Q9P2B2 & o & 2138 & RABL2A & Q9UBK7 & 1 \\
\hline 2092 & PTGR1 & Q14914 & I & 2139 & RABL6 & Q3YEC7 & 0,1 \\
\hline 2093 & PTGR2 & Q8N8N7 & I & 2140 & RAC1 & P63000 & 0,1 \\
\hline 2094 & PTMA & P06454 & o & 2141 & RAC2 & P15153 & $\mathrm{s}, \mathrm{I}$ \\
\hline 2095 & PTMS & P20962 & o & 2142 & RACK1 & P63244 & $\mathrm{I}, \mathrm{O}$ \\
\hline 2096 & PTPA & Q15257 & I & 2143 & RAD23B & P54727 & 0,1 \\
\hline 2097 & PTPN1 & P18031 & o & 2144 & RAD50 & Q92878 & I \\
\hline 2098 & PTPN11 & Q06124 & 1 & 2145 & RAl1 & Q7Z5J4 & o \\
\hline 2099 & PTPN13 & Q12923 & c & 2146 & RALA & P11233 & o \\
\hline 2100 & PTPN6 & P29350 & I & 2147 & RALGAPA1 & Q6GYQ0 & I \\
\hline 2101 & PTPRC & P08575 & 0,1 & 2148 & RALY & Q9UKM9 & 1 \\
\hline 2102 & PTPRJ & Q12913 & o & 2149 & RAN & P62826 & 1 \\
\hline 2103 & PTRHD1 & Q6GMV3 & I & 2150 & RANBP1 & P43487 & 1 \\
\hline 2104 & PUDP & Q08623 & I & 2151 & RANBP2 & P49792 & o \\
\hline 2105 & PUF60 & Q9UHX1 & 1 & 2152 & RANBP3 & Q9H6Z4 & $h, l$ \\
\hline 2106 & PURA & Q00577 & I & 2153 & RANGAP1 & P46060 & I \\
\hline 2107 & PURB & Q96QR8 & I & 2154 & RAP1A & P62834 & 0 \\
\hline
\end{tabular}




\begin{tabular}{|c|c|c|c|c|c|c|c|}
\hline & Gene & $\begin{array}{l}\text { UniProt Acces- } \\
\text { sion Number }\end{array}$ & Studies & & Gene & $\begin{array}{l}\text { UniProt Acces- } \\
\text { sion Number }\end{array}$ & Studies \\
\hline 2155 & RAP1B & P61224 & I & 2202 & RNF213 & Q63HN8 & 0,1 \\
\hline 2156 & RARRES1 & P49788 & I & 2203 & RNF40 & 075150 & I \\
\hline 2157 & RARS & P54136 & 1,0 & 2204 & $\mathrm{RNH} 1$ & P13489 & 0,1 \\
\hline 2158 & RBBP4 & Q09028 & 1,0 & 2205 & RNMT & O43148 & 1 \\
\hline 2159 & RBBP7 & Q16576 & 1 & 2206 & RNPEP & Q9H4A4 & I \\
\hline 2160 & RBBP8 & Q99708 & $b$ & 2207 & RO60 & P10155 & I \\
\hline 2161 & RBBP9 & 075884 & I & 2208 & ROCK2 & O75116 & I \\
\hline 2162 & RBKS & Q9H477 & I & 2209 & ROPN1L & Q96C74 & I \\
\hline 2163 & RBM12 & Q9NTZ6 & I & 2210 & RPA1 & P27694 & I \\
\hline 2164 & RBM14 & Q96PK6 & 0 & 2211 & RPA3 & P35244 & I \\
\hline 2165 & RBM25 & P49756 & I & 2212 & RPE & Q96AT9 & 1 \\
\hline 2166 & RBM3 & P98179 & $\mathrm{s}, \mathrm{I}$ & 2213 & RPIA & P49247 & 1 \\
\hline 2167 & RBM39 & Q14498 & 1 & 2214 & RPL10 & P27635 & 0 \\
\hline 2168 & RBM47 & A0AV96 & I & 2215 & RPL10A & P62906 & $\mathrm{I}, \mathrm{O}$ \\
\hline 2169 & RBMX & P38159 & 0,1 & 2216 & RPL10L & Q96L21 & o \\
\hline 2170 & RBP1 & P09455 & I & 2217 & RPL11 & P62913 & 1,0 \\
\hline 2171 & RBP4 & P02753 & j j, j, & 2218 & RPL12 & P30050 & 1,0 \\
\hline 2172 & $\mathrm{RCC} 1$ & P18754 & I & 2219 & RPL13 & P26373 & 1,0 \\
\hline 2173 & $\mathrm{RCC} 2$ & Q9P258 & I & 2220 & RPL13A & P40429 & I \\
\hline 2174 & RCN1 & Q15293 & 1,0 & 2221 & RPL14 & P50914 & 1,0 \\
\hline 2175 & RCN2 & Q14257 & I & 2222 & RPL15 & P61313 & 1 \\
\hline 2176 & $\mathrm{RDH} 10$ & Q8IZV5 & I & 2223 & RPL17 & P18621 & 1,0 \\
\hline 2177 & $\mathrm{RDH} 11$ & Q8TC12 & 0 & 2224 & RPL18 & Q07020 & 1,0 \\
\hline 2178 & $\mathrm{RDH} 14$ & Q9HBH5 & 0 & 2225 & RPL18A & Q02543 & $\mathrm{I}, \mathrm{O}$ \\
\hline 2179 & RDX & P35241 & 1 & 2226 & RPL19 & P84098 & 1,0 \\
\hline 2180 & RECQL & F5H2L2 & I & 2227 & RPL21 & P46778 & 0 \\
\hline 2181 & REEP5 & Q00765 & o & 2228 & RPL22 & P35268 & I \\
\hline 2182 & RELA & Q04206 & I & 2229 & RPL23 & P62829 & $\mathrm{I}, \mathrm{O}$ \\
\hline 2183 & RELCH & Q9P260 & I & 2230 & RPL23A & P62750 & 1,0 \\
\hline 2184 & RER1 & 015258 & o & 2231 & RPL24 & P83731 & $\mathrm{I}, \mathrm{O}$ \\
\hline 2185 & RETN & Q9HD89 & $r, l$ & 2232 & RPL26 & P61254 & I \\
\hline 2186 & RETREG3 & Q86VR2 & o & 2233 & RPL26L1 & Q9UNX3 & o \\
\hline 2187 & RETSAT & Q6NUM9 & o & 2234 & RPL27 & P61353 & I \\
\hline 2188 & RHBDD2 & Q6NTF9 & I & 2235 & RPL27A & P46776 & $\mathrm{I}, \mathrm{O}$ \\
\hline 2189 & $\mathrm{RHOA}$ & P61586 & I & 2236 & RPL28 & P46779 & 0 \\
\hline 2190 & $\mathrm{RHOC}$ & P08134 & I & 2237 & RPL29 & P47914 & 0 \\
\hline 2191 & RHOG & P84095 & o & 2238 & RPL3 & P39023 & 1,0 \\
\hline 2192 & RHOT2 & Q8IXI1 & 0,1 & 2239 & RPL30 & P62888 & I \\
\hline 2193 & RIDA & P52758 & $\mathrm{l}, \mathrm{g}$ & 2240 & RPL31 & P62899 & 1 \\
\hline 2194 & RMDN3 & Q96TC7 & 0,1 & 2241 & RPL32 & P62910 & 0 \\
\hline 2195 & RMND1 & Q9NWS8 & o & 2242 & RPL34 & P49207 & 0 \\
\hline 2196 & RNASE1 & P07998 & $s, r, k, l$ & 2243 & RPL35 & P42766 & 0 \\
\hline 2197 & RNASE2 & P10153 & I & 2244 & RPL35A & P18077 & o \\
\hline 2198 & RNASE3 & P12724 & $s, r, l, o$ & 2245 & RPL37A & G5E9R3 & 1 \\
\hline 2199 & RNASE4 & P34096 & 1 & 2246 & RPL38 & P63173 & I \\
\hline 2200 & RNASET2 & O00584 & 1 & 2247 & RPL4 & P36578 & $\mathrm{I}, \mathrm{O}$ \\
\hline 2201 & RNF170 & Q96K19 & o & 2248 & RPL5 & P46777 & $\mathrm{I}, \mathrm{O}$ \\
\hline
\end{tabular}




\begin{tabular}{|c|c|c|c|c|c|c|c|}
\hline & Gene & $\begin{array}{l}\text { UniProt Acces- } \\
\text { sion Number }\end{array}$ & Studies & & Gene & $\begin{array}{l}\text { UniProt Acces- } \\
\text { sion Number }\end{array}$ & Studies \\
\hline 2249 & RPL6 & Q02878 & 1,0 & 2296 & RRAS2 & P62070 & $\mathrm{o,h}$ \\
\hline 2250 & RPL7 & P18124 & $\mathrm{l}, \mathrm{O}$ & 2297 & RRBP1 & Q9P2E9 & 0,1 \\
\hline 2251 & RPL7A & P62424 & $\mathrm{l}, \mathrm{O}$ & 2298 & RSF1 & Q96T23 & k \\
\hline 2252 & RPL8 & P62917 & o & 2299 & RSL1D1 & 076021 & o \\
\hline 2253 & RPL9 & P32969 & I & 2300 & RSPH1 & Q8WYR4 & I \\
\hline 2254 & RPL9P7 & P32969 & I & 2301 & $\mathrm{RSPH} 3$ & Q86UC2 & I \\
\hline 2255 & RPL9P8 & P32969 & I & 2302 & $\mathrm{RSPH} 4 \mathrm{~A}$ & Q5TD94 & I \\
\hline 2256 & RPL9P9 & P32969 & I & 2303 & RSPH9 & Q9H1X1 & I \\
\hline 2257 & RPLPO & P05388 & o & 2304 & RSU1 & Q15404 & I \\
\hline 2258 & RPLPOP6 & Q8NHW5 & I & 2305 & RTCA & 000442 & I \\
\hline 2259 & RPLP1 & P05386 & $\mathrm{s}, \mathrm{l}$ & 2306 & RTCB & Q9Y3I0 & I \\
\hline 2260 & RPLP2 & P05387 & $\mathrm{s}, \mathrm{r}, \mathrm{l}, \mathrm{O}$ & 2307 & RTN3 & 095197 & 0,1 \\
\hline 2261 & RPN1 & P04843 & $\mathrm{l}, \mathrm{O}$ & 2308 & RTN4 & Q9NQC3 & 0,1 \\
\hline 2262 & RPN2 & P04844 & $\mathrm{l}, \mathrm{O}$ & 2309 & RTRAF & Q9Y224 & I \\
\hline 2263 & RPRD1B & Q9NQG5 & I & 2310 & RUNX2 & Q13950 & s \\
\hline 2264 & RPS10P5 & Q9NQ39 & I & 2311 & RUVBL1 & Q9Y265 & $\mathrm{s}, \mathrm{r}, \mathrm{o}, \mathrm{l}$ \\
\hline 2265 & RPS11 & P62280 & I & 2312 & RUVBL2 & Q9Y230 & 0,1 \\
\hline 2266 & RPS12 & P25398 & I & 2313 & $\mathrm{~S} 100 \mathrm{~A} 10$ & P60903 & 0,1 \\
\hline 2267 & RPS13 & P62277 & $\mathrm{l}, \mathrm{O}$ & 2314 & S100A11 & P31949 & $\mathrm{s}, \mathrm{r}, \mathrm{o}, \mathrm{e}, \mathrm{l}, \mathrm{a}$ \\
\hline 2268 & RPS14 & P62263 & $\mathrm{l}, \mathrm{O}$ & 2315 & $\mathrm{~S} 100 \mathrm{~A} 12$ & P80511 & $s, r, l$ \\
\hline 2269 & RPS15A & P62244 & $\mathrm{l}, \mathrm{O}$ & 2316 & S100A13 & Q99584 & I \\
\hline 2270 & RPS16 & P62249 & $\mathrm{l}, \mathrm{O}$ & 2317 & $\mathrm{~S} 100 \mathrm{~A} 14$ & Q9HCY8 & $\mathrm{l}, \mathrm{O}$ \\
\hline 2271 & RPS17 & P08708 & $\mathrm{l}, \mathrm{O}$ & 2318 & S100A16 & Q96FQ6 & o \\
\hline 2272 & RPS18 & P62269 & $\mathrm{l}, \mathrm{O}$ & 2319 & $\mathrm{~S} 100 \mathrm{~A} 2$ & P29034 & I \\
\hline 2273 & RPS19 & P39019 & $\mathrm{l}, \mathrm{O}$ & 2320 & S100A4 & P26447 & $\mathrm{r}, \mathrm{l}, \mathrm{o}$ \\
\hline 2274 & RPS2 & P15880 & $\mathrm{l}, \mathrm{O}$ & 2321 & S100A6 & P06703 & $\mathrm{s}, \mathrm{r}, \mathrm{o}, \mathrm{l}$ \\
\hline 2275 & RPS20 & P60866 & $\mathrm{l}, \mathrm{O}$ & 2322 & S100A7 & P31151 & $\mathrm{s}, \mathrm{r}, \mathrm{l}, \mathrm{a}$ \\
\hline 2276 & RPS21 & P63220 & $\mathrm{l}, \mathrm{O}$ & 2323 & S100A8 & P05109 & $c, f, u, s, r, n, p, t, l, l, o, a, t$ \\
\hline 2277 & RPS23 & P62266 & 0 & 2324 & S100A9 & P06702 & $c, i, f, q, s, u, r, n, l, p, o, q, a$ \\
\hline 2278 & RPS24 & P62847 & $\mathrm{l}, \mathrm{O}$ & 2325 & S100P & P25815 & $\mathrm{s}, \mathrm{r}, \mathrm{l}, \mathrm{O}$ \\
\hline 2279 & RPS25 & P62851 & $\mathrm{l}, \mathrm{O}$ & 2326 & SAA1 & PODJI8 & I \\
\hline 2280 & RPS26P11 & Q5JNZ5 & I & 2327 & SAE1 & Q9UBE0 & I \\
\hline 2281 & RPS27 & P42677 & $\mathrm{l}, \mathrm{O}$ & 2328 & SAFB & Q15424 & o \\
\hline 2282 & RPS27L & Q71UM5 & o & 2329 & SAFB2 & Q14151 & I \\
\hline 2283 & RPS28 & P62857 & $\mathrm{l}, \mathrm{O}$ & 2330 & SAG & P10523 & $\mathrm{h}$ \\
\hline 2284 & RPS3 & P23396 & $\mathrm{l}, \mathrm{O}$ & 2331 & SAMD9 & Q5K651 & I \\
\hline 2285 & RPS3A & P61247 & $\mathrm{l}, \mathrm{O}$ & 2332 & SAMHD1 & Q9Y3Z3 & $r, s, l, o$ \\
\hline 2286 & RPS4X & P62701 & $\mathrm{I}, \mathrm{O}$ & 2333 & SAMM50 & Q9Y512 & 0,1 \\
\hline 2287 & RPS5 & P46782 & I & 2334 & SAR1A & Q9NR31 & 1 \\
\hline 2288 & RPS6 & P62753 & $\mathrm{I}, \mathrm{O}$ & 2335 & SAR1B & Q9Y6B6 & I \\
\hline 2289 & RPS6KA1 & Q15418 & I & 2336 & SARG & Q9BW04 & I \\
\hline 2290 & RPS6KA3 & P51812 & I & 2337 & SARS & P49591 & I \\
\hline 2291 & RPS7 & P62081 & $\mathrm{l}, \mathrm{O}$ & 2338 & SART3 & Q15020 & I \\
\hline 2292 & RPS8 & P62241 & $\mathrm{l}, \mathrm{O}$ & 2339 & SAXO2 & Q658L1 & I \\
\hline 2293 & RPS9 & P46781 & $\mathrm{l}, \mathrm{O}$ & 2340 & SBDS & Q9Y3A5 & I \\
\hline 2294 & RPSA & P08865 & $\mathrm{l}, \mathrm{O}$ & 2341 & SCAMP1 & 015126 & o \\
\hline 2295 & RRAD & P55042 & I & 2342 & SCAMP2 & 015127 & 0,1 \\
\hline
\end{tabular}




\begin{tabular}{|c|c|c|c|c|c|c|c|}
\hline & Gene & $\begin{array}{l}\text { UniProt Acces- } \\
\text { sion Number }\end{array}$ & Studies & & Gene & $\begin{array}{l}\text { UniProt Acces- } \\
\text { sion Number }\end{array}$ & Studies \\
\hline 2343 & SCAMP3 & 014828 & o & 2390 & SEPHS2 & Q99611 & I \\
\hline 2344 & SCARB2 & Q14108 & $\mathrm{I}, \mathrm{O}$ & 2391 & SEPTIN10 & Q9P0V9 & I \\
\hline 2345 & SCCPDH & Q8NBX0 & 0,1 & 2392 & SEPTIN11 & Q9NVA2 & 1 \\
\hline 2346 & SCEL & 095171 & 0 & 2393 & SEPTIN2 & Q15019 & o,h,l \\
\hline 2347 & SCFD1 & Q8WVM8 & I & 2394 & SEPTIN6 & Q14141 & I \\
\hline 2348 & SCGB1A1 & P11684 & $q, s, r, k, q$ & 2395 & SEPTIN7 & Q16181 & I \\
\hline 2349 & SCGB1D1 & 095968 & $k, s, r$ & 2396 & SEPTIN8 & Q92599 & I \\
\hline 2350 & SCGB1D2 & 095969 & $k, s, r, l$ & 2397 & SEPTIN9 & Q9UHD8 & 0,1 \\
\hline 2351 & SCGB2A1 & 075556 & $f, s, r, k, l$ & 2398 & SERBP1 & Q8NC51 & I \\
\hline 2352 & SCGB2A2 & Q13296 & $k, b$ & 2399 & SERINC1 & Q9NRX5 & o \\
\hline 2353 & SCGN & 076038 & $\mathrm{~h}$ & 2400 & SERPINA1 & P01009 & $f, b, c, s, r, k, n, j, p, l, o, a, j$ \\
\hline 2354 & SCIN & Q9Y6U3 & I & 2401 & SERPINA3 & P01011 & $\mathrm{s}, \mathrm{r}, \mathrm{k}, \mathrm{l}, \mathrm{a}$ \\
\hline 2355 & SCLY & Q96I15 & I & 2402 & SERPINA4 & P29622 & I \\
\hline 2356 & SCP2 & P22307 & $\mathrm{I}, \mathrm{O}$ & 2403 & SERPINA6 & P08185 & 1 \\
\hline 2357 & SCPEP1 & Q9HB40 & I & 2404 & SERPINB1 & P30740 & $s, r, n, p, l, o$ \\
\hline 2358 & SCRIB & Q14160 & o & 2405 & SERPINB10 & P48595 & I \\
\hline 2359 & SCRN1 & Q12765 & I & 2406 & SERPINB12 & Q96P63 & a \\
\hline 2360 & SCRN2 & Q96FV2 & I & 2407 & SERPINB13 & Q9UIV8 & I \\
\hline 2361 & SDC1 & P18827 & 0,1 & 2408 & SERPINB2 & P05120 & $\mathrm{p}, \mathrm{l}, \mathrm{h}$ \\
\hline 2362 & SDC4 & P31431 & 0,1 & 2409 & SERPINB3 & P29508 & $s, r, b, c, m, p, l, e, o, a$ \\
\hline 2363 & SDF4 & G3V1E2 & I & 2410 & SERPINB4 & P48594 & $\mathrm{s}, \mathrm{l}, \mathrm{o}, \mathrm{a}, \mathrm{d}$ \\
\hline 2364 & SDHA & P31040 & 0,1 & 2411 & SERPINB5 & P36952 & 0,1 \\
\hline 2365 & SDHB & P21912 & I & 2412 & SERPINB6 & P35237 & I \\
\hline 2366 & SDR39U1 & Q9NRG7 & I & 2413 & SERPINB9 & P50453 & I \\
\hline 2367 & SEC11A & P67812 & 0 & 2414 & SERPINC1 & P01008 & $\mathrm{r}, \mathrm{l}$ \\
\hline 2368 & SEC11C & Q9BY50 & o & 2415 & SERPIND1 & P05546 & I \\
\hline 2369 & SEC13 & P55735 & I & 2416 & SERPINF1 & P36955 & I \\
\hline 2370 & SEC14L2 & O76054 & I & 2417 & SERPINF2 & P08697 & I \\
\hline 2371 & SEC14L3 & Q9UDX4 & I & 2418 & SERPING1 & P05155 & I \\
\hline 2372 & SEC16A & 015027 & 1,0 & 2419 & SET & Q01105 & $\mathrm{s}, \mathrm{l}, \mathrm{o}$ \\
\hline 2373 & SEC22B & O75396 & 0,1 & 2420 & $\mathrm{SF} 3 \mathrm{~A} 1$ & Q15459 & I \\
\hline 2374 & SEC23A & Q15436 & I & 2421 & SF3A3 & Q12874 & $\mathrm{I}, \mathrm{O}$ \\
\hline 2375 & SEC23B & Q15437 & I & 2422 & SF3B1 & 075533 & 0,1 \\
\hline 2376 & SEC23IP & Q9Y6Y8 & 0,1 & 2423 & SF3B2 & Q13435 & I \\
\hline 2377 & SEC24A & 095486 & I & 2424 & SF3B3 & Q15393 & $\mathrm{I}, \mathrm{O}$ \\
\hline 2378 & SEC24C & P53992 & 0,1 & 2425 & SFN & P31947 & s,n,l,o,p,a \\
\hline 2379 & SEC24D & 094855 & I & 2426 & SFPQ & P23246 & 0,1 \\
\hline 2380 & SEC31A & 094979 & I & 2427 & SFXN1 & Q9H9B4 & 0 \\
\hline 2381 & SEC61A1 & P61619 & o & 2428 & SFXN2 & Q96NB2 & 0 \\
\hline 2382 & SEC62 & Q99442 & 0 & 2429 & SFXN3 & Q9BWM7 & 1,0 \\
\hline 2383 & SEC63 & Q9UGP8 & o & 2430 & SFXN4 & Q6P4A7 & o \\
\hline 2384 & SEL1L3 & Q68CR1 & s & 2431 & SGPL1 & 095470 & 0 \\
\hline 2385 & SELENBP1 & Q13228 & $c, r, s, o, l, p, e$ & 2432 & SGTA & 043765 & I \\
\hline 2386 & SELENOS & Q9BQE4 & o & 2433 & $\mathrm{SH} 2 \mathrm{D} 4 \mathrm{~A}$ & Q9H788 & I \\
\hline 2387 & SEMG1 & P04279 & $\mathrm{a}$ & 2434 & SH3BGRL & 075368 & $\mathrm{l}, \mathrm{O}$ \\
\hline 2388 & SEMG2 & Q02383 & $\mathrm{a}$ & 2435 & SH3BGRL2 & Q9UJC5 & 1 \\
\hline 2389 & SEPHS1 & P49903 & I & 2436 & SH3BGRL3 & Q9H299 & $\mathrm{s}, \mathrm{l}$ \\
\hline
\end{tabular}




\begin{tabular}{|c|c|c|c|c|c|c|c|}
\hline & Gene & $\begin{array}{l}\text { UniProt Acces- } \\
\text { sion Number }\end{array}$ & Studies & & Gene & $\begin{array}{l}\text { UniProt Acces- } \\
\text { sion Number }\end{array}$ & Studies \\
\hline 2437 & SH3GLB1 & Q9Y371 & I & 2484 & SLC44A4 & Q53GD3 & o \\
\hline 2438 & SH3GLB2 & Q9NR46 & I & 2485 & SLC4A1 & P02730 & $\mathrm{l}, \mathrm{O}$ \\
\hline 2439 & SHMT1 & P34896 & I & 2486 & SLC4A4 & Q9Y6R1 & o \\
\hline 2440 & SHMT2 & P34897 & o & 2487 & SLC5A1 & P13866 & o \\
\hline 2441 & SHPK & Q9UHJ6 & I & 2488 & SLC6A14 & Q9UN76 & o \\
\hline 2442 & SHTN1 & A0MZ66 & I & 2489 & SLC9A1 & P19634 & o \\
\hline 2443 & SIGIRR & Q61A17 & o & 2490 & SLC9A3R1 & 014745 & $\mathrm{l}, \mathrm{O}$ \\
\hline 2444 & SKIV2L & Q15477 & I & 2491 & SLK & Q9H2G2 & I \\
\hline 2445 & SKP1 & P63208 & I & 2492 & SLPI & P03973 & $\mathrm{s}, \mathrm{r}, \mathrm{k}, \mathrm{q}, \mathrm{c}, \mathrm{l}, \mathrm{q}$ \\
\hline 2446 & SLC12A2 & P55011 & $\mathrm{l}, \mathrm{O}$ & 2493 & SMAD9 & 015198 & I \\
\hline 2447 & SLC12A6 & HOYMQ9 & $h, o$ & 2494 & SMAP2 & Q8WU79 & I \\
\hline 2448 & SLC12A7 & Q9Y666 & o & 2495 & SMARCA2 & P51531 & o \\
\hline 2449 & SLC16A1 & P53985 & o & 2496 & SMARCA5 & 060264 & o \\
\hline 2450 & SLC16A3 & 015427 & o & 2497 & SMARCC1 & Q92922 & s \\
\hline 2451 & SLC16A6 & 015403 & s & 2498 & SMC1A & Q14683 & 0,1 \\
\hline 2452 & SLC1A4 & P43007 & o & 2499 & SMC3 & Q9UQE7 & 0,1 \\
\hline 2453 & SLC1A5 & Q15758 & o & 2500 & SMPD2 & O60906 & 0,1 \\
\hline 2454 & $\mathrm{SLC} 22 \mathrm{~A} 18$ & Q96BI1 & o & 2501 & SMPD3 & Q9NY59 & o \\
\hline 2455 & SLC22A4 & Q9H015 & o & 2502 & SMPD4 & Q9NXE4 & o \\
\hline 2456 & SLC25A1 & P53007 & 0,1 & 2503 & SMR3B & P02814 & k \\
\hline 2457 & SLC25A10 & Q9UBX3 & o & 2504 & SMS & P52788 & I \\
\hline 2458 & SLC25A11 & Q02978 & $\mathrm{l}, \mathrm{O}$ & 2505 & SMU1 & Q2TAY7 & I \\
\hline 2459 & SLC25A12 & O75746 & 0,1 & 2506 & SMYD5 & Q6GMV2 & I \\
\hline 2460 & SLC25A13 & Q9UJS0 & $\mathrm{l}, \mathrm{O}$ & 2507 & SNAP23 & 000161 & o \\
\hline 2461 & SLC25A17 & O43808 & o & 2508 & SNAP91 & O60641 & I \\
\hline 2462 & SLC25A20 & O43772 & o & 2509 & SNCA & P37840 & I \\
\hline 2463 & SLC25A22 & Q9H936 & o & 2510 & SNCG & O76070 & I \\
\hline 2464 & SLC25A24 & Q6NUK1 & I & 2511 & SND1 & Q7KZF4 & 0,1 \\
\hline 2465 & SLC25A3 & Q00325 & $\mathrm{l}, \mathrm{O}$ & 2512 & SNRNP200 & 075643 & 0,1 \\
\hline 2466 & SLC25A4 & P12235 & $\mathrm{l}, \mathrm{O}$ & 2513 & SNRNP40 & Q96DI7 & I \\
\hline 2467 & SLC25A5 & P05141 & $\mathrm{I}, \mathrm{O}$ & 2514 & SNRNP70 & P08621 & 0,1 \\
\hline 2468 & SLC25A6 & P12236 & $\mathrm{I}, \mathrm{I}, \mathrm{O}, \mathrm{O}$ & 2515 & SNRPA & P09012 & I \\
\hline 2469 & SLC27A1 & Q6PCB7 & o & 2516 & SNRPA1 & P09661 & I \\
\hline 2470 & SLC27A2 & 014975 & 0,1 & 2517 & SNRPB & P14678 & I \\
\hline 2471 & SLC27A3 & Q5K4L6 & o & 2518 & SNRPD1 & P62314 & $\mathrm{s}, \mathrm{I}$ \\
\hline 2472 & SLC27A4 & Q6P1M0 & o & 2519 & SNRPD2 & P62316 & I \\
\hline 2473 & SLC2A1 & P11166 & o & 2520 & SNRPD3 & P62318 & $\mathrm{l}, \mathrm{O}$ \\
\hline 2474 & SLC2A14 & Q8TDB8 & o & 2521 & SNRPE & P62304 & 1 \\
\hline 2475 & SLC33A1 & 000400 & o & 2522 & SNRPF & P62306 & $\mathrm{l}, \mathrm{O}$ \\
\hline 2476 & SLC35A1 & P78382 & o & 2523 & SNU13 & P55769 & I \\
\hline 2477 & SLC35A3 & Q9Y2D2 & o & 2524 & SNX11 & Q9Y5W9 & $\mathrm{h}$ \\
\hline 2478 & SLC35B2 & Q8TB61 & o & 2525 & SNX16 & P57768 & $\mathrm{h}$ \\
\hline 2479 & SLC35F6 & Q8N357 & o & 2526 & SNX2 & 060749 & I \\
\hline 2480 & SLC37A4 & 043826 & o & 2527 & SNX3 & 060493 & I \\
\hline 2481 & SLC3A2 & P08195 & o & 2528 & SNX5 & Q9Y5X3 & I \\
\hline 2482 & SLC44A1 & Q8WWI5 & o & 2529 & SNX6 & Q9UNH7 & I \\
\hline 2483 & SLC44A2 & Q8IWA5 & o & 2530 & SOAT1 & P35610 & o \\
\hline
\end{tabular}




\begin{tabular}{|c|c|c|c|c|c|c|c|}
\hline & Gene & $\begin{array}{l}\text { UniProt Acces- } \\
\text { sion Number }\end{array}$ & Studies & & Gene & $\begin{array}{l}\text { UniProt Acces- } \\
\text { sion Number }\end{array}$ & Studies \\
\hline 2531 & SOD1 & P00441 & $c, s, r, o, j, p, l, j$ & 2578 & SSR4 & P51571 & 0,1 \\
\hline 2532 & SOD2 & P04179 & $m, m, o, o, l, l$ & 2579 & SSRP1 & Q08945 & I \\
\hline 2533 & SOD3 & P08294 & $h, I$ & 2580 & ST13 & P50502 & 0,1 \\
\hline 2534 & SORD & Q00796 & $r, o, l$ & 2581 & ST14 & Q9Y5Y6 & o \\
\hline 2535 & SORL1 & Q92673 & o & 2582 & ST3GAL4 & Q11206 & o \\
\hline 2536 & SP100 & P23497 & I & 2583 & STARD10 & Q9Y365 & I \\
\hline 2537 & SPA17 & Q15506 & I & 2584 & STAT1 & P42224 & I \\
\hline 2538 & SPACA9 & Q96E40 & I & 2585 & STAT2 & P52630 & 1 \\
\hline 2539 & SPAG6 & 075602 & 0,1 & 2586 & STAT3 & P40763 & 0,1 \\
\hline 2540 & SPAG9 & O60271 & I & 2587 & STAT6 & P42226 & I \\
\hline 2541 & SPATA18 & Q8TC71 & 0,1 & 2588 & STATH & P02808 & $\mathrm{s}, \mathrm{r}, \mathrm{f}, \mathrm{i}, \mathrm{l}$ \\
\hline 2542 & SPATA6 & Q9NWH7 & 1 & 2589 & STAU1 & 095793 & 1 \\
\hline 2543 & SPCS1 & Q9Y6A9 & o & 2590 & STIM1 & Q13586 & o \\
\hline 2544 & SPCS2 & Q15005 & o & 2591 & STIM2 & Q9P246 & o \\
\hline 2545 & SPCS3 & P61009 & o & 2592 & STIP1 & P31948 & $c, 0, l$ \\
\hline 2546 & SPEN & Q96T58 & o & 2593 & STK24 & Q9Y6E0 & I \\
\hline 2547 & SPNS1 & Q9H2V7 & $b$ & 2594 & STK33 & Q9BYT3 & I \\
\hline 2548 & SPR & P35270 & I & 2595 & STK38 & Q15208 & I \\
\hline 2549 & SPRR1A & P35321 & $\mathrm{r}, \mathrm{l}, \mathrm{a}$ & 2596 & STK39 & Q9UEW8 & I \\
\hline 2550 & SPRR1B & P22528 & $\mathrm{s}, \mathrm{r}, \mathrm{l}, \mathrm{a}$ & 2597 & STK4 & Q13043 & $\mathrm{h}$ \\
\hline 2551 & SPRR2B & P35325 & $\mathrm{r}, \mathrm{a}$ & 2598 & STMN1 & P16949 & I \\
\hline 2552 & SPRR3 & Q9UBC9 & $\mathrm{r}, \mathrm{l}$ & 2599 & STOM & P27105 & o \\
\hline 2553 & SPTAN1 & Q13813 & $\mathrm{I}, \mathrm{O}$ & 2600 & STOML2 & Q9UJZ1 & 0,1 \\
\hline 2554 & SPTBN1 & Q01082 & 0,1 & 2601 & STOML3 & Q8TAV4 & o \\
\hline 2555 & SPTBN2 & 015020 & $\mathrm{I}, \mathrm{O}$ & 2602 & STRAP & Q9Y3F4 & I \\
\hline 2556 & SQOR & Q9Y6N5 & 0,1 & 2603 & STS & P08842 & o \\
\hline 2557 & SRI & P30626 & $\mathrm{I}, \mathrm{m}$ & 2604 & STT3A & P46977 & $\mathrm{I}, \mathrm{O}$ \\
\hline 2558 & SRP14 & P37108 & I & 2605 & STT3B & Q8TCJ2 & o \\
\hline 2559 & SRP68 & Q9UHB9 & 0,1 & 2606 & STX11 & 075558 & $\mathrm{~h}$ \\
\hline 2560 & SRP72 & 076094 & I & 2607 & STX12 & Q86Y82 & o \\
\hline 2561 & SRPRA & P08240 & o & 2608 & STX18 & Q9P2W9 & o \\
\hline 2562 & SRPRB & Q9Y5M8 & o & 2609 & STX4 & Q12846 & o \\
\hline 2563 & SRRM1 & Q8IYB3 & 1 & 2610 & STX5 & Q13190 & o \\
\hline 2564 & SRRM2 & Q9UQ35 & o & 2611 & STX7 & 015400 & o \\
\hline 2565 & SRRT & Q9BXP5 & 1 & 2612 & STX8 & Q9UNKO & o \\
\hline 2566 & SRSF1 & Q07955 & $\mathrm{I}, \mathrm{O}$ & 2613 & STXBP3 & 000186 & o \\
\hline 2567 & SRSF10 & O75494 & I & 2614 & SUB1 & P53999 & I \\
\hline 2568 & SRSF11 & Q05519 & I & 2615 & SUCLA2 & Q9P2R7 & $0, I$ \\
\hline 2569 & SRSF2 & Q01130 & $\mathrm{I}, \mathrm{O}$ & 2616 & SUCLG1 & P53597 & 0,1 \\
\hline 2570 & SRSF3 & P84103 & 0,1 & 2617 & SUCLG2 & Q96199 & 0,1 \\
\hline 2571 & SRSF5 & Q13243 & 1 & 2618 & SUGT1 & Q9Y2Z0 & I \\
\hline 2572 & SRSF7 & Q16629 & $\mathrm{I}, \mathrm{O}$ & 2619 & SULT1A2 & P50226 & I \\
\hline 2573 & SSB & P05455 & 0,1 & 2620 & SULT1A3 & PODMM9 & I \\
\hline 2574 & SSBP1 & Q04837 & I & 2621 & SULT2A 1 & Q06520 & $\mathrm{h}$ \\
\hline 2575 & SSH3 & Q8TE77 & 1 & 2622 & SUMF2 & Q8NBJ7 & I \\
\hline 2576 & SSR1 & P43307 & 0,1 & 2623 & SUN1 & 094901 & o \\
\hline 2577 & SSR3 & Q9UNL2 & o & 2624 & SUN2 & Q9UH99 & o \\
\hline
\end{tabular}




\begin{tabular}{|c|c|c|c|c|c|c|c|}
\hline & Gene & $\begin{array}{l}\text { UniProt Acces- } \\
\text { sion Number }\end{array}$ & Studies & & Gene & $\begin{array}{l}\text { UniProt Acces- } \\
\text { sion Number }\end{array}$ & Studies \\
\hline 2625 & SURF1 & Q15526 & o & 2672 & TFG & Q92734 & I \\
\hline 2626 & SURF4 & 015260 & $\mathrm{l}, \mathrm{O}$ & 2673 & TFRC & P02786 & o \\
\hline 2627 & SVIL & 095425 & s & 2674 & TGM2 & P21980 & $\mathrm{I}, \mathrm{O}$ \\
\hline 2628 & SWAP70 & Q9UH65 & I & 2675 & TGM3 & Q08188 & $\mathrm{p}, \mathrm{a}$ \\
\hline 2629 & SYNCRIP & O60506 & $\mathrm{I}, \mathrm{O}$ & 2676 & TGOLN2 & O43493 & o \\
\hline 2630 & SYNE1 & Q8NF91 & $b, l, o$ & 2677 & THOP1 & P52888 & I \\
\hline 2631 & SYNE2 & Q8WXH0 & 0,1 & 2678 & THRAP3 & Q9Y2W1 & 0,1 \\
\hline 2632 & SYNGR1 & O43759 & o & 2679 & THUMPD1 & Q9NXG2 & 1 \\
\hline 2633 & SYNGR2 & O43760 & o & 2680 & THYN1 & Q9P016 & I \\
\hline 2634 & SYNJ2BP & P57105 & o & 2681 & TIGAR & Q9NQ88 & I \\
\hline 2635 & SYPL1 & Q16563 & I & 2682 & TIMM17B & 060830 & o \\
\hline 2636 & SYTL1 & Q8IYJ3 & I & 2683 & TIMM50 & Q3ZCQ8 & $\mathrm{I}, \mathrm{O}$ \\
\hline 2637 & SYVN1 & Q86TM6 & o & 2684 & TIMMDC1 & Q9NPL8 & o \\
\hline 2638 & TACC2 & O95359 & I & 2685 & TIMP1 & P01033 & $s, r, k, l$ \\
\hline 2639 & TACSTD2 & P09758 & 0,1 & 2686 & TIPRL & O75663 & I \\
\hline 2640 & TAF15 & Q92804 & I & 2687 & TJP2 & Q9UDY2 & 0,1 \\
\hline 2641 & TAGLN2 & P37802 & $s, r, o, l$ & 2688 & TJP3 & 095049 & 0,1 \\
\hline 2642 & TALDO1 & P37837 & $\mathrm{s}, \mathrm{r}, \mathrm{l}, \mathrm{O}$ & 2689 & TKFC & Q3LXA3 & I \\
\hline 2643 & TAOK1 & Q7L7X3 & g & 2690 & TKT & P29401 & $s, r, k, o, l, g$ \\
\hline 2644 & TAP1 & Q03518 & o & 2691 & TLN1 & Q9Y490 & $\mathrm{I}, \mathrm{O}$ \\
\hline 2645 & TAP2 & Q03519 & $\mathrm{l}, \mathrm{O}$ & 2692 & TLR3 & 015455 & o \\
\hline 2646 & TAPBP & 015533 & 0,1 & 2693 & TM7SF2 & 076062 & o \\
\hline 2647 & TAPBPL & Q9BX59 & o & 2694 & TM9SF1 & 015321 & o \\
\hline 2648 & TARDBP & Q13148 & I & 2695 & TM9SF2 & Q99805 & 0,1 \\
\hline 2649 & TARS & P26639 & I & 2696 & TM9SF3 & Q9HD45 & 0,1 \\
\hline 2650 & TAX1BP3 & 014907 & I & 2697 & TM9SF4 & Q92544 & o \\
\hline 2651 & TBC1D1 & Q86TI0 & $\mathrm{l}, \mathrm{O}$ & 2698 & TMBIM6 & P55061 & o \\
\hline 2652 & TBC1D15 & Q8TC07 & I & 2699 & TMCO1 & Q9UM00 & o \\
\hline 2653 & TBCA & 075347 & I & 2700 & TMED1 & Q13445 & o \\
\hline 2654 & TBCB & Q99426 & I & 2701 & TMED10 & P49755 & $\mathrm{I}, \mathrm{O}$ \\
\hline 2655 & TBCD & Q9BTW9 & I & 2702 & TMED2 & Q15363 & o \\
\hline 2656 & TBL2 & Q9Y4P3 & o & 2703 & TMED4 & Q7Z7H5 & o \\
\hline 2657 & TCERG1 & 014776 & I & 2704 & TMED7 & Q9Y3B3 & $\mathrm{l}, \mathrm{O}$ \\
\hline 2658 & TCF3 & P15923 & $i, f$ & 2705 & TMED9 & Q9BVK6 & 0,1 \\
\hline 2659 & TCIRG1 & Q13488 & o & 2706 & TMEM109 & Q9BVC6 & o \\
\hline 2660 & TCN1 & P20061 & $b, s, r, k, l$ & 2707 & TMEM126A & Q9H061 & o \\
\hline 2661 & TCOF1 & Q13428 & o & 2708 & TMEM128 & Q5BJH2 & o \\
\hline 2662 & TCP1 & P17987 & 0,1 & 2709 & TMEM14C & Q9P0S9 & o \\
\hline 2663 & TECR & Q9NZ01 & 0,1 & 2710 & TMEM165 & Q9HC07 & o \\
\hline 2664 & TEKT1 & Q969V4 & I & 2711 & TMEM173 & Q86WV6 & $\mathrm{l}, \mathrm{O}$ \\
\hline 2665 & TEKT2 & Q9UIF3 & I & 2712 & TMEM200C & A6NKL6 & s \\
\hline 2666 & TERT & 014746 & $\mathrm{~h}$ & 2713 & TMEM205 & Q6UW68 & I \\
\hline 2667 & TES & Q9UGI8 & I & 2714 & TMEM231 & Q9H6L2 & I \\
\hline 2668 & TEX10 & Q9NXF1 & o & 2715 & TMEM245 & Q9H330 & o \\
\hline 2669 & TF & P02787 & $s, r, k, q, i, b, c, p, l, l, o, a, q$ & 2716 & TMEM259 & Q4ZIN3 & o \\
\hline 2670 & TFAM & Q00059 & I & 2717 & TMEMЗОА & Q9NV96 & o \\
\hline 2671 & TFF3 & Q07654 & $s, r, l$ & 2718 & TMEMЗОВ & Q3MIR4 & o \\
\hline
\end{tabular}




\begin{tabular}{|c|c|c|c|c|c|c|c|}
\hline & Gene & $\begin{array}{l}\text { UniProt Acces- } \\
\text { sion Number }\end{array}$ & Studies & & Gene & $\begin{array}{l}\text { UniProt Acces- } \\
\text { sion Number }\end{array}$ & Studies \\
\hline 2719 & TMEM33 & P57088 & o & 2766 & TRAM1 & Q15629 & 0 \\
\hline 2720 & TMEM63A & 094886 & 0 & 2767 & TRANK1 & 015050 & I \\
\hline 2721 & TMEM63B & Q5T3F8 & 0 & 2768 & TRAPPC3 & 043617 & I \\
\hline 2722 & TMEM65 & Q6PI78 & 0 & 2769 & TRBC1 & P01850 & $\mathrm{h}$ \\
\hline 2723 & TMEM67 & Q5HYA8 & 0 & 2770 & TRBV16 & A0A087WV62 & $\mathrm{h}$ \\
\hline 2724 & TMOD3 & Q9NYL9 & I & 2771 & TRIM2 & Q9C040 & I \\
\hline 2725 & TMPO & P42166 & 0,1 & 2772 & TRIM25 & Q14258 & 1 \\
\hline 2726 & TMPRSS11D & 060235 & 0 & 2773 & TRIM28 & Q13263 & $\mathrm{I}, \mathrm{O}$ \\
\hline 2727 & TMPRSS4 & Q9NRS4 & 0 & 2774 & TRIM29 & Q14134 & 0,1 \\
\hline 2728 & TMSB4X & P62328 & $\mathrm{s}, \mathrm{r}, \mathrm{l}, \mathrm{O}$ & 2775 & TRIM56 & Q9BRZ2 & 0 \\
\hline 2729 & TMTC3 & Q6ZXV5 & 0 & 2776 & TRIP11 & Q15643 & 0 \\
\hline 2730 & TMUB1 & Q9BVT8 & 0 & 2777 & TRIP12 & Q14669 & 0 \\
\hline 2731 & TMX1 & Q9H3N1 & 0,1 & 2778 & TRIP6 & Q15654 & I \\
\hline 2732 & TMX3 & Q96JJ7 & 0 & 2779 & TRMT112 & Q9UI30 & 1 \\
\hline 2733 & TMX4 & Q9H1E5 & o & 2780 & TRMT12 & Q53H54 & $\mathrm{m}$ \\
\hline 2734 & TNC & P24821 & I & 2781 & TRMT61A & Q96FX7 & I \\
\hline 2735 & TNFAIP8 & 095379 & I & 2782 & TRPM4 & Q8TD43 & o \\
\hline 2736 & TNIK & Q9UKE5 & I & 2783 & TSC22D1 & Q15714 & I \\
\hline 2737 & TNPO1 & Q92973 & I & 2784 & TSG101 & Q99816 & I \\
\hline 2738 & TNXB & P22105 & s & 2785 & TSGA10 & Q9BZW7 & I \\
\hline 2739 & TOM1 & 060784 & I & 2786 & TSN & Q15631 & I \\
\hline 2740 & TOMM22 & Q9NS69 & 0 & 2787 & TSNAX & Q99598 & I \\
\hline 2741 & тОММ34 & Q15785 & I & 2788 & TSPAN1 & 060635 & $\mathrm{~s}, \mathrm{I}$ \\
\hline 2742 & TOMM40 & 096008 & 0 & 2789 & TSPAN3 & 060637 & o \\
\hline 2743 & TOMM70 & 094826 & 1,0 & 2790 & TST & Q16762 & 0,1 \\
\hline 2744 & TOP2B & Q02880 & 0 & 2791 & TSTA3 & Q13630 & $1, p$ \\
\hline 2745 & TOR1AIP1 & Q5JTV8 & 0,1 & 2792 & TSTD1 & Q8NFU3 & I \\
\hline 2746 & TP53І3 & Q53FA7 & I & 2793 & TTBK1 & Q5TCY1 & $b$ \\
\hline 2747 & TP53RK & Q96S44 & I & 2794 & TTC21A & Q8NDW8 & I \\
\hline 2748 & TPBG & Q13641 & 0 & 2795 & TTC25 & Q96NG3 & I \\
\hline 2749 & TPD52 & P55327 & I & 2796 & TTC26 & A0AVF1 & I \\
\hline 2750 & TPD52L2 & O43399 & I & 2797 & TTLL12 & Q14166 & 1 \\
\hline 2751 & TPI1 & P60174 & $\mathrm{n}, \mathrm{c}, \mathrm{s}, \mathrm{r}, \mathrm{l}, \mathrm{o}, \mathrm{a}$ & 2798 & TTN & Q8WZ42 & $s, r, o, l$ \\
\hline 2752 & TPM1 & P09493 & $0, j, p, j$ & 2799 & TTR & P02766 & $r, s, i, l, j, j, j$ \\
\hline 2753 & TPM3 & P06753 & $\mathrm{c}, \mathrm{r}, \mathrm{s}, \mathrm{O}, \mathrm{m}, \mathrm{l}$ & 2800 & TUBA1A & Q71U36 & $\mathrm{s}, \mathrm{l}, \mathrm{o}, \mathrm{a}$ \\
\hline 2754 & TPM4 & P67936 & o,p,l & 2801 & TUBA1B & P68363 & $\mathrm{s}, \mathrm{r}, \mathrm{c}, \mathrm{m}, \mathrm{I}$ \\
\hline 2755 & TPMT & P51580 & I & 2802 & TUBA1C & Q9BQE3 & $c, m$ \\
\hline 2756 & TPP1 & 014773 & 0,1 & 2803 & TUBA4A & P68366 & $c, l$ \\
\hline 2757 & TPP2 & P29144 & I & 2804 & TUBA8 & Q9NY65 & C \\
\hline 2758 & TPPP & 094811 & I & 2805 & TUBB & P07437 & $\mathrm{s}, \mathrm{c}, \mathrm{m}, \mathrm{l}, \mathrm{O}$ \\
\hline 2759 & TPPP3 & Q9BW30 & $r, s, o, l$ & 2806 & TUBB1 & Q9H4B7 & o \\
\hline 2760 & TPR & P12270 & I & 2807 & TUBB2B & Q9BVA1 & I \\
\hline 2761 & TPRKB & Q9Y3C4 & I & 2808 & TUBB3 & Q13509 & I \\
\hline 2762 & TPT1 & P13693 & $j, p, l, o, j$ & 2809 & TUBB4A & P04350 & $\mathrm{s}, \mathrm{I}$ \\
\hline 2763 & TRA2A & Q13595 & I & 2810 & TUBB4B & P68371 & $r, s, o$ \\
\hline 2764 & TRA2B & P62995 & I & 2811 & TUBB6 & Q9BUF5 & I \\
\hline 2765 & TRADD & Q15628 & I & 2812 & TUBB8 & Q3ZCM7 & 1 \\
\hline
\end{tabular}




\begin{tabular}{|c|c|c|c|c|c|c|c|}
\hline & Gene & $\begin{array}{l}\text { UniProt Acces- } \\
\text { sion Number }\end{array}$ & Studies & & Gene & $\begin{array}{l}\text { UniProt Acces- } \\
\text { sion Number }\end{array}$ & Studies \\
\hline 2813 & TUFM & P49411 & $\mathrm{c}, \mathrm{m}, \mathrm{l}, \mathrm{O}$ & 2860 & UGDH & 060701 & $\mathrm{~s}, \mathrm{o}, \mathrm{l}$ \\
\hline 2814 & TUSC3 & Q13454 & o & 2861 & UGGT1 & Q9NYU2 & 0,1 \\
\hline 2815 & TWF1 & Q12792 & I & 2862 & UGP2 & Q16851 & I \\
\hline 2816 & TWF2 & Q6IBS0 & I & 2863 & UGT1A6 & P19224 & o \\
\hline 2817 & TXN & P10599 & s,r,o,l,p,e & 2864 & UGT2A1 & Q9Y4X1 & 0,1 \\
\hline 2818 & TXN2 & Q99757 & I & 2865 & UMPS & P11172 & I \\
\hline 2819 & TXNDC12 & 095881 & I & 2866 & UNC93B1 & Q9H1C4 & o \\
\hline 2820 & TXNDC17 & Q9BRA2 & I & 2867 & UPF1 & Q92900 & I \\
\hline 2821 & TXNDC5 & Q8NBS9 & j j, j & 2868 & UQCR10 & Q9UDW1 & I \\
\hline 2822 & TXNL1 & O43396 & I & 2869 & UQCRB & P14927 & $\mathrm{I}, \mathrm{O}$ \\
\hline 2823 & TXNRD1 & Q16881 & $r, s, I$ & 2870 & UQCRC1 & P31930 & $c, l, o, j, j$ \\
\hline 2824 & TXNRD2 & Q9NNW7 & I & 2871 & UQCRC2 & P22695 & $\mathrm{I}, \mathrm{O}$ \\
\hline 2825 & TYMP & P19971 & $s, r, o, l$ & 2872 & UQCRFS1 & P47985 & o \\
\hline 2826 & U2AF1 & Q01081 & o & 2873 & UQCRFS1P1 & P0C7P4 & I \\
\hline 2827 & U2AF1L4 & Q8WU68 & I & 2874 & UQCRH & P07919 & I \\
\hline 2828 & U2AF2 & P26368 & I & 2875 & UQCRQ & 014949 & o \\
\hline 2829 & UAP1 & Q16222 & I & 2876 & URB2 & Q14146 & b \\
\hline 2830 & UBA1 & P22314 & $\mathrm{s}, \mathrm{r}, \mathrm{o}, \mathrm{l}$ & 2877 & UROD & P06132 & I \\
\hline 2831 & UBA2 & Q9UBT2 & I & 2878 & USO1 & 060763 & I \\
\hline 2832 & UBA3 & Q8TBC4 & I & 2879 & USP14 & P54578 & I \\
\hline 2833 & UBA5 & Q9GZZ9 & I & 2880 & USP5 & P45974 & $\mathrm{l}, \mathrm{t}, \mathrm{o}, \mathrm{t}$ \\
\hline 2834 & UBA52 & P62987 & I & 2881 & USP7 & Q93009 & I \\
\hline 2835 & UBA6 & A0AVT1 & I & 2882 & USP9X & Q93008 & $\mathrm{I}, \mathrm{O}$ \\
\hline 2836 & UBC & POCG48 & $\mathrm{s}, \mathrm{O}, \mathrm{a}$ & 2883 & UTRN & P46939 & 0,1 \\
\hline 2837 & UBE2A & P49459 & I & 2884 & VAMP2 & P63027 & o \\
\hline 2838 & UBE2D3 & P61077 & I & 2885 & VAMP8 & Q9BV40 & 0,1 \\
\hline 2839 & UBE2I & P63279 & I & 2886 & VANGL1 & Q8TAA9 & o \\
\hline 2840 & UBE2K & P61086 & I & 2887 & VAPA & Q9P0L0 & 0,1 \\
\hline 2841 & UBE2L3 & P68036 & I & 2888 & VAPB & 095292 & I \\
\hline 2842 & UBE2L6 & 014933 & I & 2889 & VARS & P26640 & $\mathrm{I}, \mathrm{O}$ \\
\hline 2843 & UBE2M & P61081 & 1 & 2890 & VASP & P50552 & 0,1 \\
\hline 2844 & UBE2N & P61088 & 0,1 & 2891 & VAT1 & Q99536 & $\mathrm{I}, \mathrm{O}$ \\
\hline 2845 & UBE2O & Q9C0C9 & I & 2892 & VBP1 & P61758 & I \\
\hline 2846 & UBE2V1 & Q13404 & I & 2893 & VCL & P18206 & 0,1 \\
\hline 2847 & UBLCP1 & Q8WVY7 & I & 2894 & VCP & P55072 & $\mathrm{r}, \mathrm{c}, \mathrm{s}, \mathrm{l}, \mathrm{O}$ \\
\hline 2848 & UBQLN1 & Q9UMX0 & I & 2895 & VDAC1 & P21796 & 0,1 \\
\hline 2849 & UBR4 & Q5T4S7 & I & 2896 & VDAC2 & P45880 & 0,1 \\
\hline 2850 & UBXN1 & Q04323 & I & 2897 & VDAC3 & Q9Y277 & 0,1 \\
\hline 2851 & UBXN11 & Q5T124 & I & 2898 & VILL & 015195 & I \\
\hline 2852 & UBXN4 & Q92575 & o & 2899 & VIM & P08670 & $\mathrm{s}, \mathrm{r}, \mathrm{o}, \mathrm{l}, \mathrm{a}$ \\
\hline 2853 & UBXN6 & Q9BZV1 & I & 2900 & VMO1 & Q7Z5L0 & I \\
\hline 2854 & UCHL3 & P15374 & I & 2901 & VPS13C & Q709C8 & $\mathrm{I}, \mathrm{O}$ \\
\hline 2855 & UCHL5 & Q9Y5K5 & I & 2902 & VPS26A & 075436 & I \\
\hline 2856 & UFC1 & Q9Y3C8 & I & 2903 & VPS29 & Q9UBQ0 & I \\
\hline 2857 & UFD1 & Q92890 & I & 2904 & VPS35 & Q96QK1 & I \\
\hline 2858 & UFL1 & O94874 & $\mathrm{l}, \mathrm{O}$ & 2905 & VPS36 & Q86VN1 & I \\
\hline 2859 & UFM1 & P61960 & I & 2906 & VPS4A & Q9UN37 & I \\
\hline
\end{tabular}




\begin{tabular}{|c|c|c|c|c|c|c|c|}
\hline & Gene & $\begin{array}{l}\text { UniProt Acces- } \\
\text { sion Number }\end{array}$ & Studies & & Gene & $\begin{array}{l}\text { UniProt Acces- } \\
\text { sion Number }\end{array}$ & Studies \\
\hline 2907 & VPS4B & 075351 & I & 2935 & YARS2 & Q9Y2Z4 & 0 \\
\hline 2908 & VPS9D1 & Q9Y2B5 & 0 & 2936 & YBX1 & P67809 & 0 \\
\hline 2909 & VTA1 & Q9NP79 & I & 2937 & YBX3 & P16989 & 0,1 \\
\hline 2910 & VTI1B & Q9UEU0 & 0 & 2938 & YIF1A & 095070 & 0 \\
\hline 2911 & VTN & P04004 & I & 2939 & YIPF3 & Q9GZM5 & o \\
\hline 2912 & VWA5A & 000534 & I & 2940 & YIPF6 & Q96EC8 & 0 \\
\hline 2913 & WARS & P23381 & $r, l, p, o$ & 2941 & YME1L1 & Q96TA2 & 0 \\
\hline 2914 & WASF2 & Q9Y6W5 & I & 2942 & YWHAB & P31946 & $\mathrm{s}, \mathrm{O}$ \\
\hline 2915 & WASHC2A & Q641Q2 & I & 2943 & YWHAE & P62258 & $s, 0, l, p$ \\
\hline 2916 & WASHC4 & Q2M389 & I & 2944 & YWHAG & P61981 & $s, 0, I$ \\
\hline 2917 & WDR1 & 075083 & 0,1 & 2945 & YWHAH & Q04917 & 0,1 \\
\hline 2918 & WDR13 & Q9H1Z4 & I & 2946 & YWHAQ & P27348 & $\mathrm{s}, \mathrm{l}, \mathrm{O}$ \\
\hline 2919 & WDR54 & Q9H977 & I & 2947 & YWHAZ & P63104 & $\mathrm{s}, \mathrm{r}, \mathrm{o}, \mathrm{l}, \mathrm{p}, \mathrm{a}$ \\
\hline 2920 & WDR61 & Q9GZS3 & I & 2948 & ZC3H15 & Q8WU90 & I \\
\hline 2921 & WDR77 & Q9BQA1 & I & 2949 & ZDHHC13 & Q8IUH4 & 0 \\
\hline 2922 & WDR92 & Q96MX6 & I & 2950 & ZFAND1 & Q8TCF1 & I \\
\hline 2923 & WFDC2 & Q14508 & $\mathrm{s}, \mathrm{r}, \mathrm{k}, \mathrm{l}$ & 2951 & ZFP2 & Q6ZN57 & $\mathrm{m}$ \\
\hline 2924 & WFS1 & 076024 & 0 & 2952 & ZG16B & Q96DA0 & $k, b, s, r, l$ \\
\hline 2925 & WHAMM & Q8TF30 & I & 2953 & ZMPSTE24 & 075844 & 1,0 \\
\hline 2926 & WRB & 000258 & 0 & 2954 & ZMYND10 & 075800 & I \\
\hline 2927 & $\mathrm{XDH}$ & P47989 & I & 2955 & ZNF106 & Q9H2Y7 & s \\
\hline 2928 & XPNPEP1 & Q9NQW7 & I & 2956 & ZNF165 & P49910 & s \\
\hline 2929 & XPO1 & 014980 & I & 2957 & ZNF185 & 015231 & o,l \\
\hline 2930 & XPO7 & Q9UIA9 & I & 2958 & ZNF207 & 043670 & I \\
\hline 2931 & XRCC5 & P13010 & 0,1 & 2959 & ZNF263 & 014978 & s \\
\hline 2932 & XRCC6 & P12956 & 0,1 & 2960 & ZNF609 & 015014 & $\mathrm{~g}$ \\
\hline 2933 & XRN2 & Q9H0D6 & I & 2961 & ZSCAN31 & Q96LW9 & $\mathrm{h}$ \\
\hline 2934 & YARS & P54577 & I & 2962 & ZW10 & O43264 & I \\
\hline
\end{tabular}

Studies.

\begin{tabular}{|c|c|c|}
\hline & Author & Title \\
\hline a & Benson et al. & $\begin{array}{l}\text { Extensive fractionation and identification of Proteins within nasal lavage fluids from allergic rhinitis and asthmatic } \\
\text { chronic rhinosinusitis patients }\end{array}$ \\
\hline $\mathrm{b}$ & Casado et al. & Identification of human nasal mucous proteins using proteomics \\
\hline c & Debat et al. & Identification of human olfactory cleft mucus proteins using proteomic analysis \\
\hline d & Farajzadeh Deroee et al. & Regression of polypoid nasal mucosa after systemic corticosteroid therapy: a proteomics study \\
\hline e & Gelardi et al. & Proteomic analysis of human nasal mucosa: different expression profile in rhino-pathologic states \\
\hline$f$ & Ghafouri et al. & Comparative proteomics of nasal fluid in seasonal allergic rhinitis \\
\hline g & Kim et al. & Fatty acid binding protein 1 is related with development of aspirin-exacerbated respiratory disease \\
\hline $\mathrm{h}$ & Lee et al. & $\begin{array}{l}\text { Proteomic analysis of normal human nasal mucosa: Establishment of a two-dimensional electrophoresis reference } \\
\text { map }\end{array}$ \\
\hline i & Lindahl et al. & $\begin{array}{l}\text { Nasal lavage fluid and proteomics as means to identify the effects of the irritating epoxy chemical dimethylben- } \\
\text { zylamine }\end{array}$ \\
\hline j & Min-Man et al. & Differential proteomic analysis of nasal polyps, chronic sinusitis, and normal nasal mucosa tissues \\
\hline k & Mortstedt et al. & Screening method using selected reaction monitoring for targeted proteomics studies of nasal lavage fluid \\
\hline
\end{tabular}




\begin{tabular}{|c|c|c|}
\hline & Author & Title \\
\hline I & Ndika et al. & $\begin{array}{l}\text { Epithelial proteome profiling suggests the essential role of interferon-inducible proteins in patients with allergic } \\
\text { rhinitis }\end{array}$ \\
\hline $\mathrm{m}$ & Roxo-Rosa et al. & $\begin{array}{l}\text { Proteomic analysis of nasal cells from cystic fibrosis patients and non-cystic fibrosis control individuals: search for } \\
\text { novel biomarkers of cystic fibrosis lung disease }\end{array}$ \\
\hline $\mathrm{n}$ & Schoenebeck et al. & Improved preparation of nasal lavage fluid (NLF) as a noninvasive sample for proteomic biomarker discovery \\
\hline o & Simoes et al. & Molecular profiling of the human nasal epithelium: A proteomics approach \\
\hline $\mathrm{p}$ & Suojalehto et al. & Nasal protein profiles in work-related asthma caused by different exposures \\
\hline q & Tewfik et al. & Proteomics of nasal mucus in chronic rhinosinusitis \\
\hline r & Tomazic et al. & $\begin{array}{l}\text { Nasal mucus proteomic changes reflect altered immune responses and epithelial permeability in patients with } \\
\text { allergic rhinitis }\end{array}$ \\
\hline s & Tomazic et al. & $\begin{array}{l}\text { Seasonal proteome changes of nasal mucus reflect perennial inflammatory response and reduced defence mecha- } \\
\text { nisms and plasticity in allergic rhinitis }\end{array}$ \\
\hline $\mathrm{t}$ & Upton et al. & Chronic Rhinosinusitis With Nasal Polyps: A Proteomic Analysis \\
\hline $\mathrm{u}$ & Wahlen et al. & $\begin{array}{l}\text { Protein profiles of nasal lavage fluid from individuals with work-related upper airway symptoms associated with } \\
\text { moldy and damp buildings }\end{array}$ \\
\hline
\end{tabular}

Supplement IV: Study demographics.

\begin{tabular}{|c|c|c|c|c|c|}
\hline Author & Patient selection & Diagnostic criteria & $\begin{array}{l}\text { Co- } \\
\text { morbidities }\end{array}$ & Smoking & Oral steroid \\
\hline Benson et al. & NR & NR & Asthma & 0 & 0 \\
\hline Casado et al. & Outpatient Clinic & $\begin{array}{l}\text { Clinical symptoms (Facial pain, tenderness, } \\
\text { mucopurulent discharge) }\end{array}$ & 0 & NR & 0 \\
\hline Debat et al. & Hospital Clinic & Nasoendoscopy & 0 & 0 & 0 \\
\hline Ghafouri et al. & NR & $\begin{array}{l}\text { Nasal symptoms scores } \\
\text { Nasoendoscopy }\end{array}$ & 0 & 0 & 0 \\
\hline Lindahl et al. & NR & Nasoendoscopy & $\begin{array}{l}\text { DMBA expo- } \\
\text { sure }\end{array}$ & 0 & NR \\
\hline Mortstedt et al. & NR & NR & NR & NR & NR \\
\hline Schoenebeck et al. & NR & NR & 0 & NR & NR \\
\hline Tewfik et al. & Outpatient Clinic & $\begin{array}{l}\text { Nasoendoscopy } \\
\text { CT Sinuses (Sinus Health Allergy Partnership criteria) }\end{array}$ & 0 & 0 & 0 \\
\hline Tomazic et al. & NR & Nasoendoscopy (EPOS Criteria) & 0 & NR & 0 \\
\hline Tomazic et al. & NR & Nasoendoscopy (EPOS Criteria) & 0 & 0 & 0 \\
\hline Wahlen et al. & NR & NR & 0 & 0 & NR \\
\hline Farajzadeh Deroee et al. & Hospital Clinic & Nasoendoscopy & 0 & 0 & 3 \\
\hline Gelardi et al. & Hospital Clinic & $\begin{array}{l}\text { Clinical symptoms } \\
\text { Nasoendoscopy }\end{array}$ & 0 & NR & NR \\
\hline Kim et al. & NR & $\begin{array}{l}\text { Clinical symptoms } \\
\text { Nasoendoscopy } \\
\text { CT Sinuses }\end{array}$ & $\begin{array}{l}\text { Asthma } \\
\text { ATA } 8 \\
\text { AERD } 5\end{array}$ & ATA 2 & 0 \\
\hline Lee et al. & NR & $\begin{array}{l}\text { Clinical symptoms } \\
\text { Nasoendoscopy }\end{array}$ & 0 & NR & 0 \\
\hline Min-Man et al. & Hospital Clinic & $\begin{array}{l}\text { Nasoendoscopy } \\
\text { CT Sinuses }\end{array}$ & 0 & NR & 0 \\
\hline Ndika J et al. & Hospital Clinic & Seasonal allergy symptoms questionnaire & 0 & 0 & NR \\
\hline Roxo-Rosa et al. & NR & Clinical symptoms & 0 & 0 & 0 \\
\hline Simoes et al. & NR & NR & NR & NR & NR \\
\hline Suojalehto et al. & Hospital Clinic & NR & 0 & 0 & NR \\
\hline Upton DC et al. & Outpatient Clinic & $\begin{array}{l}\text { Nasoendoscopy } \\
\text { CT Sinuses (AAO-HNS CRS TF criteria) }\end{array}$ & NR & 0 & 3 \\
\hline
\end{tabular}

AAO-HNS: American Academy of Otolaryngology-Head and Neck Surgery Chronic Rhinosinusitis Task Force; ATA: Aspirin-tolerant asthma; AERD: Aspirin-exacerbated respiratory disease; DMBA: Dimethylbenzylamine; EPOS: European Position Paper on Chronic Rhinosinusitis and Nasal Polyps; NR: Not reported. 
Supplement V: Cellular pathways/ cellular components/ biological processes/ molecular functions / cellular pathways.

\begin{tabular}{|c|c|c|c|c|}
\hline Pathway & Classification & Source & $\begin{array}{l}\text { Adjusted } \\
\text { P-value }\end{array}$ & Genes \\
\hline $\begin{array}{l}\text { Antigen activates B Cell Receptor (BCR) leading } \\
\text { to generation of second messengers_Homo } \\
\text { sapiens_R-HSA-983695 }\end{array}$ & Immune system & Mucus CRS & 0.00148 & $\begin{array}{l}\text { IGHM;IGKC;IGHV3- } \\
\text { 23;IGLC1;CALM1 }\end{array}$ \\
\hline Apoptosis_Homo sapiens_R-HSA-109581 & $\begin{array}{l}\text { Programmed cell } \\
\text { death }\end{array}$ & Mucus CRS & 0.00152 & $\begin{array}{l}\text { DSP;UBC;LMNA;PKP1;DSG1;SF } \\
\text { N;VIM;YWHAZ }\end{array}$ \\
\hline $\begin{array}{l}\text { Apoptotic cleavage of cell adhesion proteins_ } \\
\text { Homo sapiens_R-HSA-351906 }\end{array}$ & $\begin{array}{l}\text { Programmed cell } \\
\text { death }\end{array}$ & Mucus CRS & 0.00373 & DSP;DSG1;PKP1 \\
\hline $\begin{array}{l}\text { Apoptotic cleavage of cellular proteins_Homo } \\
\text { sapiens_R-HSA-111465 }\end{array}$ & $\begin{array}{l}\text { Programmed cell } \\
\text { death }\end{array}$ & Mucus CRS & 0.00054 & DSP;LMNA;DSG1;PKP1;VIM \\
\hline $\begin{array}{l}\text { Apoptotic execution phase_Homo sapiens_R- } \\
\text { HSA-75153 }\end{array}$ & $\begin{array}{l}\text { Programmed cell } \\
\text { death }\end{array}$ & Mucus CRS & 0.00199 & DSP;LMNA;DSG1;PKP1;VIM \\
\hline $\begin{array}{l}\text { Binding and Uptake of Ligands by Scavenger } \\
\text { Receptors_Homo sapiens_R-HSA-2173782 }\end{array}$ & $\begin{array}{l}\text { Vesicle-mediated } \\
\text { transport }\end{array}$ & Mucus CRS & 0.00000 & $\begin{array}{l}\text { COL1A1;COL1A2;IGKC;IGHV3- } \\
\text { 23;HP;IGLC1;HBA1;HPR;IGHA2 }\end{array}$ \\
\hline $\begin{array}{l}\text { Binding and Uptake of Ligands by Scavenger } \\
\text { Receptors_Homo sapiens_R-HSA-2173782 }\end{array}$ & $\begin{array}{l}\text { Vesicle-mediated } \\
\text { transport }\end{array}$ & Mucus Healthy + CRS & 0.00017 & IGKV1-5;ALB;HBB;IGHA1 \\
\hline $\begin{array}{l}\text { CD22 mediated BCR regulation_Homo } \\
\text { sapiens_R-HSA-5690714 }\end{array}$ & Immune system & Mucus CRS & 0.00059 & IGHM;IGKC;IGHV3-23;IGLC1 \\
\hline $\begin{array}{l}\text { Classical antibody-mediated complement acti- } \\
\text { vation_Homo sapiens_R-HSA-173623 }\end{array}$ & Immune system & Mucus CRS & 0.00001 & $\begin{array}{l}\text { IGHG3;IGHG4;IGHG1;IGHG2;IG } \\
\text { KC;IGHV3-23;IGLC1 }\end{array}$ \\
\hline $\begin{array}{l}\text { Complement cascade_Homo sapiens_R- } \\
\text { HSA-166658 }\end{array}$ & Immune system & Mucus CRS & 0.00017 & $\begin{array}{l}\text { IGHG3;IGHG4;IGHG1;IGHG2;IG } \\
\text { KC;IGHV3-23;IGLC1 }\end{array}$ \\
\hline $\begin{array}{l}\text { Creation of C4 and C2 activators_Homo } \\
\text { sapiens_R-HSA-166786 }\end{array}$ & Immune system & Mucus CRS & 0.00001 & $\begin{array}{l}\text { IGHG3;IGHG4;IGHG1;IGHG2;IG } \\
\text { KC;IGHV3-23;IGLC1 }\end{array}$ \\
\hline $\begin{array}{l}\text { Detoxification of Reactive Oxygen Species_ } \\
\text { Homo sapiens_R-HSA-3299685 }\end{array}$ & $\begin{array}{l}\text { Cellular responses } \\
\text { to external stimuli }\end{array}$ & Mucosa Healthy + CRS & 0.02341 & PRDX2;CAT;SOD1 \\
\hline $\begin{array}{l}\text { Diseases associated with visual transduction_ } \\
\text { Homo sapiens_R-HSA-2474795 }\end{array}$ & Disease & Mucosa Healthy + CRS & 0.02021 & RBP4;TTR \\
\hline $\begin{array}{l}\text { FCERI mediated Ca+2 mobilization_Homo } \\
\text { sapiens_R-HSA-2871809 }\end{array}$ & Immune system & Mucus CRS & 0.04948 & IGKC;IGHV3-23;IGLC1;CALM1 \\
\hline $\begin{array}{l}\text { Fcgamma receptor (FCGR) dependent phagocy- } \\
\text { tosis_Homo sapiens_R-HSA-2029480 }\end{array}$ & Immune system & Mucus CRS & 0.00003 & $\begin{array}{l}\text { IGHG3;IGHG4;IGHG1 } \\
\text {;IGHG2;IGKC;IGHV3- } \\
\text { 23;MYH9;IGLC1;ACTB }\end{array}$ \\
\hline FCGR activation_Homo sapiens_R-HSA-2029481 & Immune system & Mucus CRS & 0.00001 & $\begin{array}{l}\text { IGHG3;IGHG4;IGHG1;IGHG2;IG } \\
\text { KC;IGHV3-23;IGLC1 }\end{array}$ \\
\hline Gluconeogenesis_Homo sapiens_R-HSA-70263 & Metabolism & Mucus CRS & 0.00462 & TPI1;ENO1;ALDOA;GAPDH \\
\hline $\begin{array}{l}\text { Glucose metabolism_Homo sapiens_R- } \\
\text { HSA-70326 }\end{array}$ & Metabolism & Mucus CRS & 0.00017 & $\begin{array}{l}\text { TPI1;PKM;UBC;ENO1;ALDOA;C } \\
\text { ALM1;GAPDH }\end{array}$ \\
\hline Glycolysis_Homo sapiens_R-HSA-70171 & Metabolism & Mucus CRS & 0.00028 & $\begin{array}{l}\text { TPI1;PKM;ENO1;ALDOA; } \\
\text { GAPDH }\end{array}$ \\
\hline $\begin{array}{l}\text { Initial triggering of complement_Homo } \\
\text { sapiens_R-HSA-166663 }\end{array}$ & Immune system & Mucus CRS & 0.00003 & $\begin{array}{l}\text { IGHG3;IGHG4;IGHG1;|GHG2; } \\
\text { IGKC;IGHV3-23;IGLC1 }\end{array}$ \\
\hline $\begin{array}{l}\text { Metabolism of carbohydrates_Homo sapiens_R- } \\
\text { HSA-71387 }\end{array}$ & Metabolism & Mucus CRS & 0.04885 & $\begin{array}{l}\text { TPI1;PKM;AMY2B;UBC;ENO1; } \\
\text { CALM1;ALDOA;GAPDH }\end{array}$ \\
\hline $\begin{array}{l}\text { Metabolism of fat-soluble vitamins_Homo } \\
\text { sapiens_R-HSA- } 6806667\end{array}$ & Metabolism & Mucosa Healthy + CRS & 0.02495 & RBP4;TTR;APOA1 \\
\hline Metabolism_Homo sapiens_R-HSA-1430728 & Metabolism & Mucosa Healthy + CRS & 0.00556 & $\begin{array}{l}\text { ECHS1;APOA1;NME4;PDHB;C } \\
\text { A1;RBP4;TTR;NDUFS8;ALDH1 } \\
\text { A1;CAT;CMPK1;UQCRC1;PSM } \\
\text { E1;GAPDH }\end{array}$ \\
\hline $\begin{array}{l}\text { Platelet degranulation_Homo sapiens_R- } \\
\text { HSA-114608 }\end{array}$ & Homeostasis & Mucosa Healthy + CRS & 0.02114 & SERPINA1;FGG;APOA1;SOD1 \\
\hline $\begin{array}{l}\text { Platelet degranulation_Homo sapiens_R- } \\
\text { HSA-114608 }\end{array}$ & Homeostasis & Mucus CRS & 0.04631 & $\begin{array}{l}\text { SERPINA3;SERPINA1;AHSG;CA } \\
\text { LM1;ALDOA }\end{array}$ \\
\hline
\end{tabular}




\begin{tabular}{|c|c|c|c|c|}
\hline Pathway & Classification & Source & $\begin{array}{l}\text { Adjusted } \\
\text { P-value }\end{array}$ & Genes \\
\hline $\begin{array}{l}\text { Platelet degranulation_Homo sapiens_R- } \\
\text { HSA-114608 }\end{array}$ & Homeostasis & Mucus Healthy + CRS & 0.03116 & ORM1;TF;ALB \\
\hline $\begin{array}{l}\text { Programmed Cell Death_Homo sapiens_R- } \\
\text { HSA-5357801 }\end{array}$ & $\begin{array}{l}\text { Programmed cell } \\
\text { death }\end{array}$ & Mucus CRS & 0.00155 & $\begin{array}{l}\text { DSP;UBC;LMNA;PKP1;DSG1;SF } \\
\text { N;VIM;YWHAZ }\end{array}$ \\
\hline $\begin{array}{l}\text { Regulation of actin dynamics for phagocytic cup } \\
\text { formation_Homo sapiens_R-HSA-2029482 }\end{array}$ & Immune system & Mucus CRS & 0.00001 & $\begin{array}{l}\text { IGHG3;IGHG4;IGHG1 } \\
\text {;IGHG2;IGKC;IGHV3- } \\
\text { 23;MYH9;IGLC1;ACTB }\end{array}$ \\
\hline $\begin{array}{l}\text { Response to elevated platelet cytosolic Ca2+_ } \\
\text { Homo sapiens_R-HSA-76005 }\end{array}$ & Homeostasis & Mucosa Healthy + CRS & 0.01902 & SERPINA1;FGG;APOA1;SOD1 \\
\hline $\begin{array}{l}\text { Response to elevated platelet cytosolic Ca2+__ } \\
\text { Homo sapiens_R-HSA-76005 }\end{array}$ & Homeostasis & Mucus Healthy + CRS & 0.02685 & ORM1;TF;ALB \\
\hline $\begin{array}{l}\text { Retinoid cycle disease events_Homo sapiens_R- } \\
\text { HSA-2453864 }\end{array}$ & Disease & Mucosa Healthy + CRS & 0.02274 & RBP4;TTR \\
\hline $\begin{array}{l}\text { Retinoid metabolism and transport_Homo } \\
\text { sapiens_R-HSA-975634 }\end{array}$ & $\begin{array}{l}\text { Metabolism, Sig- } \\
\text { nal transduction }\end{array}$ & Mucosa Healthy + CRS & 0.01948 & RBP4;TTR;APOA1 \\
\hline $\begin{array}{l}\text { RHO GTPases activate PKNs_Homo sapiens_R- } \\
\text { HSA-5625740 }\end{array}$ & $\begin{array}{l}\text { Signal transduc- } \\
\text { tion }\end{array}$ & Mucus CRS & 0.04735 & HIST1H4A;MYH9;SFN;YWHAZ \\
\hline $\begin{array}{l}\text { Role of phospholipids in phagocytosis_Homo } \\
\text { sapiens_R-HSA-2029485 }\end{array}$ & Immune system & Mucus CRS & 0.00003 & $\begin{array}{l}\text { IGHG3;IGHG4;IGHG 1;IGHG2;IG } \\
\text { KC;IGHV3-23;IGLC1 }\end{array}$ \\
\hline $\begin{array}{l}\text { Scavenging of heme from plasma_Homo } \\
\text { sapiens_R-HSA-2168880 }\end{array}$ & $\begin{array}{l}\text { Vesicle-mediated } \\
\text { transport }\end{array}$ & Mucus CRS & 0.00001 & $\begin{array}{l}\text { IGKC;IGHV3- } \\
\text { 23;HP;IGLC1;HPR;HBA1;IGHA2 }\end{array}$ \\
\hline $\begin{array}{l}\text { Scavenging of heme from plasma_Homo } \\
\text { sapiens_R-HSA-2168880 }\end{array}$ & $\begin{array}{l}\text { Vesicle-mediated } \\
\text { transport }\end{array}$ & Mucus Healthy + CRS & 0.00005 & IGKV1-5;ALB;HBB;IGHA1 \\
\hline $\begin{array}{l}\text { Signal Transduction_Homo sapiens_R- } \\
\text { HSA-162582 }\end{array}$ & $\begin{array}{l}\text { Signal transduc- } \\
\text { tion }\end{array}$ & Mucosa Healthy + CRS & 0.01752 & $\begin{array}{l}\text { ANXA1;FGG;PEBP1;APOA } 1 ; P D \\
\text { HB;ACTB;ACTG1;ADCYAP1;RB } \\
\text { P4;TTR;ARHGDIA;ARHGDIB;AL } \\
\text { DH1A1;PSME1 }\end{array}$ \\
\hline $\begin{array}{l}\text { Translocation of GLUT4 to the plasma mem- } \\
\text { brane_Homo sapiens_R-HSA-1445148 }\end{array}$ & $\begin{array}{l}\text { Vesicle-mediated } \\
\text { transport }\end{array}$ & Mucus CRS & 0.04940 & MYH13;SFN;CALM1;YWHAZ \\
\hline $\begin{array}{l}\text { Vesicle-mediated transport_Homo sapiens_R- } \\
\text { HSA-5653656 }\end{array}$ & $\begin{array}{l}\text { Vesicle-mediated } \\
\text { transport }\end{array}$ & Mucus CRS & 0.00017 & $\begin{array}{l}\text { SERPINA1;IGHV3-23;HP;HPR;H } \\
\text { BA1;YWHAZ;COL1A1;COL1A2; } \\
\text { IGKC;UBC;MYH13;SFN;IGLC1;C } \\
\text { ALM1;IGHA2 }\end{array}$ \\
\hline
\end{tabular}

Cellular components.

\begin{tabular}{|c|c|c|c|c|}
\hline Cellular Component & Classification & Source & $\begin{array}{l}\text { Adjusted } \\
\text { P-value }\end{array}$ & Genes \\
\hline actin filament (GO:0005884) & Cytoskeleton & Mucosa Healthy + CRS & 0.01684 & ANXA1;TPM1;ACTG1 \\
\hline azurophil granule (GO:0042582) & Secretory granule & Mucus CRS & 0.00049 & $\begin{array}{l}\text { SERPINB3;SERPINA3;PIGR;ANXA2;FAB } \\
\text { P5;DEFA1;S100A7;HRNR }\end{array}$ \\
\hline azurophil granule lumen (GO:0035578) & Secretory granule & Mucus CRS & 0.00014 & $\begin{array}{l}\text { SERPINB3;SERPINA3;ANXA2;FABP5;D } \\
\text { EFA1;S100A7;HRNR }\end{array}$ \\
\hline cytoplasmic vesicle lumen (GO:0060205) & $\begin{array}{l}\text { Cytoplasmic } \\
\text { vesicle }\end{array}$ & Mucus CRS & 0.00000 & $\begin{array}{l}\text { EEF1A1;SERPINA3;CSTB;PKM;AHSG;G } \\
\text { STP1;HBA1;ALDOA;S100A8;S100A11 }\end{array}$ \\
\hline cytoplasmic vesicle lumen (GO:0060205) & $\begin{array}{l}\text { Cytoplasmic } \\
\text { vesicle }\end{array}$ & Mucus Healthy + CRS & 0.00735 & TF;HBB;S100A9 \\
\hline cytoplasmic vesicle lumen (GO:0060205) & $\begin{array}{l}\text { Cytoplasmic } \\
\text { vesicle }\end{array}$ & Mucosa Healthy + CRS & 0.01417 & CAT;APOA1;S100A8;HSP90B1 \\
\hline cytoskeleton (GO:0005856) & Cytoskeleton & Mucus CRS & 0.00232 & $\begin{array}{l}\text { KRT4;JUP;HSPB1;KRT77;ACTB;TUBA1 } \\
\text { A;KRT17;KRT16;MYH9;VIM;ALDOA;G } \\
\text { APDH;S100A8 }\end{array}$ \\
\hline cytoskeleton (GO:0005856) & Cytoskeleton & Mucosa Healthy + CRS & 0.00089 & $\begin{array}{l}\text { ARHGDIA;ARHGDIB;TPM1;KRT8;GAP } \\
\text { DH;ACTB;S100A8;ACTG1 }\end{array}$ \\
\hline endocytic vesicle lumen (GO:0071682) & $\begin{array}{l}\text { Cytoplasmic } \\
\text { vesicle }\end{array}$ & Mucus Healthy + CRS & 0.00593 & HBB;LTF \\
\hline
\end{tabular}




\begin{tabular}{|c|c|c|c|c|}
\hline Cellular Component & Classification & Source & $\begin{array}{l}\text { Adjusted } \\
\text { P-value }\end{array}$ & Genes \\
\hline endocytic vesicle lumen (GO:0071682) & $\begin{array}{l}\text { Cytoplasmic } \\
\text { vesicle }\end{array}$ & Mucosa Healthy + CRS & 0.03186 & APOA1;HSP90B1 \\
\hline ficolin-1-rich granule (GO:0101002) & Secretory granule & Mucus CRS & 0.00000 & $\begin{array}{l}\text { EEF1A1;DSP;CSTB;SERPINB12;SERPIN } \\
\text { A1;PKM;CALML5;JUP;GSTP1;PKP1;D } \\
\text { SG1;ALDOA }\end{array}$ \\
\hline ficolin-1-rich granule lumen (GO:1904813) & Secretory granule & Mucus CRS & 0.00012 & $\begin{array}{l}\text { EEF1A1;CSTB;SERPINA1;PKM;CALML } \\
\text { 5;JUP;GSTP1;ALDOA }\end{array}$ \\
\hline ficolin-1-rich granule membrane (GO:0101003) & Secretory granule & Mucus CRS & 0.02500 & DSP;SERPINB12;DSG1;PKP1 \\
\hline focal adhesion (GO:0005925) & Adheren junction & Mucus CRS & 0.02430 & $\begin{array}{l}\text { ANXA1;JUP;HSPB1;MYH9;VIM;B2M;Y } \\
\text { WHAZ;ACTB;S100A7 }\end{array}$ \\
\hline focal adhesion (GO:0005925) & Adheren junction & Mucosa CRS & 0.00632 & HSPA9;ACTR3;HSPA5;IQGAP1;P4HB \\
\hline focal adhesion (GO:0005925) & Adheren junction & Mucosa Healthy + CRS & 0.03570 & ANXA1;CAT;ACTB;HSP90B1;ACTG1 \\
\hline intermediate filament (GO:0005882) & Cytoskeleton & Mucus CRS & 0.00000 & $\begin{array}{l}\text { KRT82;FLG;DSP;KRT4;KRT3; } \\
\text { KRT2;KRT7;KRT5;KRT10;KRT76; } \\
\text { KRT20;KRT84;KRT16;KRT14;PKP1;VI } \\
\text { M;KRT6A }\end{array}$ \\
\hline $\begin{array}{l}\text { intermediate filament cytoskeleton } \\
\text { (GO:0045111) }\end{array}$ & Cytoskeleton & Mucus CRS & 0.00000 & $\begin{array}{l}\text { DSP;FLG;KRT4;KRT3;KRT2;KRT7;KRT5; } \\
\text { KRT76;KRT10;KRT20;KRT17;KRT16;KR } \\
\text { T14;PKP1;VIM;S100A8;KRT6A }\end{array}$ \\
\hline keratin filament (GO:0045095) & Cytoskeleton & Mucus CRS & 0.00000 & KRT82;KRT4;KRT3;KRT14;KRT5;KRT84 \\
\hline mitochondrial matrix (GO:0005759) & Mitochondria & Mucosa CRS & 0.00631 & HSPA9;ALDH4A1;RIDA;CPS1;HSPD1 \\
\hline mitochondrion (GO:0005739) & Mitochondria & Mucosa Healthy + CRS & 0.04277 & $\begin{array}{l}\text { ECHS1;NDUFS8;CAT;UQCRC1;NME4;P } \\
\text { DHB;PARK7;SOD1 }\end{array}$ \\
\hline platelet alpha granule lumen (GO:0031093) & Secretory granule & Mucus CRS & 0.03316 & SERPINA3;SERPINA1;AHSG;ALDOA \\
\hline polymeric cytoskeletal fiber (GO:0099513) & Cytoskeleton & Mucus CRS & 0.00000 & $\begin{array}{l}\text { FLG;DSP;ANXA1;KRT4;KRT3;KRT2;KRT } \\
\text { 7;KRT5;KRT76;KRT10;KRT20;TUBA1A; } \\
\text { KRT16;KRT14;PKP1;VIM;KRT6A }\end{array}$ \\
\hline polymeric cytoskeletal fiber (GO:0099513) & Cytoskeleton & Mucosa Healthy + CRS & 0.04082 & ANXA1;TPM1;KRT8;ACTG1 \\
\hline secretory granule lumen (GO:0034774) & Secretory granule & Mucus CRS & 0.00000 & $\begin{array}{l}\text { SERPINB3;SERPINA3;CSTB;SERPINA1; } \\
\text { JUP;ANXA2;AHSG;GSTP1;DEFA1;HRN } \\
\text { R;EEF1A1;PKM;FABP5;ALDOA;B2M;S1 } \\
00 A 8 ; S 100 A 11 ; S 100 A 7\end{array}$ \\
\hline secretory granule lumen (GO:0034774) & Secretory granule & Mucus Healthy + CRS & 0.00000 & ORM1;TF;SLPI;ALB;LYZ;S100A9;LTF \\
\hline secretory granule lumen (GO:0034774) & Secretory granule & Mucosa Healthy + CRS & 0.00073 & $\begin{array}{l}\text { SERPINA } 1 ; \text { TTR;FGG;CAT;APOA } 1 ; \text { S100 } \\
\text { A8;TXNDC5 }\end{array}$ \\
\hline specific granule (GO:0042581) & Secretory granule & Mucus Healthy + CRS & 0.00057 & ORM1;SLPI;LYZ;LTF \\
\hline specific granule lumen (GO:0035580) & Secretory granule & Mucus Healthy + CRS & 0.00002 & ORM1;SLPI;LYZ;LTF \\
\hline tertiary granule (GO:0070820) & Secretory granule & Mucus CRS & 0.00470 & $\begin{array}{l}\text { DSP;CSTB;SERPINB12;PKP1;DSG1;AL } \\
\text { DOA;B2M }\end{array}$ \\
\hline tertiary granule (GO:0070820) & Secretory granule & Mucus Healthy + CRS & 0.00051 & ORM1;HBB;LYZ;LTF \\
\hline tertiary granule lumen (GO:1904724) & Secretory granule & Mucus Healthy + CRS & 0.00002 & ORM1;HBB;LYZ;LTF \\
\hline vacuolar lumen (GO:0005775) & Vacuole & Mucus CRS & 0.00454 & $\begin{array}{l}\text { SERPINB3;SERPINA3;ANXA2;FABP5;D } \\
\text { EFA1;S100A7;HRNR }\end{array}$ \\
\hline
\end{tabular}

\section{Biological pathways}

\begin{tabular}{|c|c|c|c|c|}
\hline Biological Process & Classification & Source & $\begin{array}{l}\text { Adjusted } \\
\text { P-value }\end{array}$ & Genes \\
\hline $\begin{array}{l}\text { 4-hydroxyproline metabolic process } \\
\text { (GO:0019471) }\end{array}$ & $\begin{array}{l}\text { Cellular metabolic } \\
\text { process }\end{array}$ & Mucosa CRS & 0.02989 & ALDH4A1;P4HB \\
\hline antibacterial humoral response (GO:0019731) & $\begin{array}{l}\text { Immune system } \\
\text { process }\end{array}$ & Mucus CRS & 0.00004 & $\begin{array}{l}\text { IGHM;BPIFA1;SEMG2;SEMG1;DEFA1 } \\
\text {;IGHA2 }\end{array}$ \\
\hline antibacterial humoral response (GO:0019731) & $\begin{array}{l}\text { Immune system } \\
\text { process }\end{array}$ & Mucus Healthy + CRS & 0.00003 & SLPI;IGHA1;JCHAIN;LTF \\
\hline
\end{tabular}




\begin{tabular}{|c|c|c|c|c|}
\hline Biological Process & Classification & Source & $\begin{array}{l}\text { Adjusted } \\
\text { P-value }\end{array}$ & Genes \\
\hline $\begin{array}{l}\text { antigen receptor-mediated signaling pathway } \\
\text { (GO:0050851) }\end{array}$ & $\begin{array}{l}\text { Immune system } \\
\text { process }\end{array}$ & Mucus CRS & 0.00144 & $\begin{array}{l}\text { IGHG3;IGHG4;IGHM;IGHG1;IGHG2;IG } \\
\text { KC;IGHV3-23;UBC;IGLC1;IGHA2 }\end{array}$ \\
\hline $\begin{array}{l}\text { antimicrobial humoral immune response me- } \\
\text { diated by antimicrobial peptide (GO:0061844) }\end{array}$ & $\begin{array}{l}\text { Immune system } \\
\text { process }\end{array}$ & Mucus CRS & 0.00020 & $\begin{array}{l}\text { BPIFA1;SEMG1;DEFA1;GAPDH;KRT6 } \\
\text { A;S100A7 }\end{array}$ \\
\hline ATP generation from ADP (GO:0006757) & $\begin{array}{l}\text { Cellular metabolic } \\
\text { process }\end{array}$ & Mucus CRS & 0.00269 & LDHA;TPI1;PKM;ALDOA \\
\hline $\begin{array}{l}\text { B cell receptor signaling pathway } \\
\text { (GO:0050853) }\end{array}$ & $\begin{array}{l}\text { Immune system } \\
\text { process }\end{array}$ & Mucus CRS & 0.00001 & $\begin{array}{l}\text { IGHG3;IGHG4;IGHM;IGHG 1;IGHG2;IG } \\
\text { KC;IGHV3-23;IGLC1;IGHA2 }\end{array}$ \\
\hline canonical glycolysis (GO:0061621) & Metabolic process & Mucus CRS & 0.00016 & TPI1;PKM;ENO1;ALDOA;GAPDH \\
\hline carbohydrate catabolic process (GO:0016052) & Metabolic process & Mucus CRS & 0.01105 & LDHA;TPI1;PKM;ALDOA \\
\hline $\begin{array}{l}\text { cellular protein metabolic process } \\
\text { (GO:0044267) }\end{array}$ & $\begin{array}{l}\text { Cellular metabolic } \\
\text { process }\end{array}$ & Mucus Healthy + CRS & 0.01029 & TF;BPIFB2;ALB;LYZ;LTF \\
\hline $\begin{array}{l}\text { cellular response to oxidative stress } \\
\text { (GO:0034599) }\end{array}$ & $\begin{array}{l}\text { Cellular response } \\
\text { to chemical } \\
\text { stimulus }\end{array}$ & Mucosa Healthy + CRS & 0.00231 & PRDX2;TPM1;CAT;PARK7;SOD1 \\
\hline cellular response to superoxide (GO:0071451) & $\begin{array}{l}\text { Cellular reponse to } \\
\text { chemical stimulus }\end{array}$ & Mucosa Healthy + CRS & 0.04045 & PRDX2;SOD1 \\
\hline $\begin{array}{l}\text { complement activation, classical pathway } \\
\text { (GO:0006958) }\end{array}$ & $\begin{array}{l}\text { Immune system } \\
\text { process }\end{array}$ & Mucus CRS & 0.00004 & $\begin{array}{l}\text { IGHG3;IGHG4;IGHM;IGHG1;IGHG2;IG } \\
\text { KC;IGHV3-23;IGLC1;IGHA2 }\end{array}$ \\
\hline defense response to bacterium (GO:0042742) & $\begin{array}{l}\text { Reponse to } \\
\text { stimulus }\end{array}$ & Mucus CRS & 0.00000 & $\begin{array}{l}\text { IGHM;IGHV3-23;DEFA1;IGHG3;IGHG4; } \\
\text { IGHG1;BPIFA1;IGHG2;IGKC;SEMG2;PR } \\
\text { B3;SEMG1;IGLC1;IGHA2;LACRT;S100A } \\
\text { 8;KRT6A;S100A7 }\end{array}$ \\
\hline defense response to bacterium (GO:0042742) & $\begin{array}{l}\text { Reponse to } \\
\text { stimulus }\end{array}$ & Mucus Healthy + CRS & 0.00002 & SLPI;LYZ;IGHA1;S100A9;JCHAIN;LTF \\
\hline defense response to fungus (GO:0050832) & $\begin{array}{l}\text { Reponse to } \\
\text { stimulus }\end{array}$ & Mucus Healthy + CRS & 0.02586 & S100A9;LTF \\
\hline $\begin{array}{l}\text { detection of chemical stimulus involved } \\
\text { in sensory perception of bitter taste } \\
\text { (GO:0001580) }\end{array}$ & $\begin{array}{l}\text { Sensory percep- } \\
\text { tion }\end{array}$ & Mucus CRS & 0.01812 & PIGR;AZGP1;PIP;CST4 \\
\hline $\begin{array}{l}\text { detection of chemical stimulus involved in } \\
\text { sensory perception of taste (GO:0050912) }\end{array}$ & $\begin{array}{l}\text { Sensory percep- } \\
\text { tion }\end{array}$ & Mucus CRS & 0.02456 & PIGR;AZGP1;PIP;CST4 \\
\hline endocytosis (GO:0006897) & Localisation & Mucus CRS & 0.04151 & $\begin{array}{l}\text { ANXA1;IGKC;AHSG;IGHV3- } \\
\text { 23;IGLC1;HPR;HBA1;IGHA2 }\end{array}$ \\
\hline endocytosis (GO:0006897) & Localisation & Mucus Healthy + CRS & 0.00066 & IGKV1-5;ALB;HBB;IGHA1;JCHAIN \\
\hline epidermal cell differentiation (GO:0009913) & $\begin{array}{l}\text { Antomical struc- } \\
\text { ture development }\end{array}$ & Mucus CRS & 0.00000 & $\begin{array}{l}\text { DSP;FLG;CSTA;ANXA1;KRT16;KRT10 } \\
\text {;SPRR2B;SPRR1A;TGM3;IVL;SPRR1B; } \\
\text { S100A7 }\end{array}$ \\
\hline epidermis development (GO:0008544) & $\begin{array}{l}\text { Antomical struc- } \\
\text { ture development }\end{array}$ & Mucus CRS & 0.00000 & $\begin{array}{l}\text { DSP;FLG;CALML5;KRT2;KRT34;KRT5;K } \\
\text { RT32;KRT31;KRT85;KRT9;KRT83;HRN } \\
\text { R;CASP14;KRT17;KABP5;KRT16;KRT14 } \\
\text {;SPRR2B;SPRR1A;SPRR1B;S100A7 }\end{array}$ \\
\hline establishment of skin barrier (GO:0061436) & $\begin{array}{l}\text { Antomical struc- } \\
\text { ture development }\end{array}$ & Mucus CRS & 0.00059 & FLG;KRT16;SFN;HRNR \\
\hline $\begin{array}{l}\text { Fc receptor mediated stimulatory signaling } \\
\text { pathway (GO:0002431) }\end{array}$ & $\begin{array}{l}\text { Immune system } \\
\text { process }\end{array}$ & Mucus CRS & 0.00063 & $\begin{array}{l}\text { IGHG3;IGHG4;IGHG1;IGHG2;IGKC;IG } \\
\text { HV3-23;IGLC1;ACTB }\end{array}$ \\
\hline $\begin{array}{l}\text { Fc-gamma receptor signaling pathway } \\
\text { (GO:0038094) }\end{array}$ & $\begin{array}{l}\text { Immune system } \\
\text { process }\end{array}$ & Mucus CRS & 0.00062 & $\begin{array}{l}\text { IGHG3;IGHG4;IGHG1;IGHG2;IGKC;IG } \\
\text { HV3-23;IGLC1;ACTB }\end{array}$ \\
\hline $\begin{array}{l}\text { Fc-gamma receptor signaling pathway in- } \\
\text { volved in phagocytosis (GO:0038096) }\end{array}$ & $\begin{array}{l}\text { Immune system } \\
\text { process }\end{array}$ & Mucus CRS & 0.00060 & $\begin{array}{l}\text { IGHG3;IGHG4;IGHG1;IGHG2;IGKC;IG } \\
\text { HV3-23;IGLC1;ACTB }\end{array}$ \\
\hline glomerular filtration (GO:0003094) & System process & Mucus Healthy + CRS & 0.01092 & IGHA1;JCHAIN \\
\hline gluconeogenesis (GO:0006094) & Metabolic process & Mucus CRS & 0.01912 & TPI1;ENO1;ALDOA;GAPDH \\
\hline $\begin{array}{l}\text { glucose catabolic process to pyruvate } \\
\text { (GO:0061718) }\end{array}$ & Metabolic process & Mucus CRS & 0.00015 & TPI1;PKM;ENO1;ALDOA;GAPDH \\
\hline glycolytic process (GO:0006096) & $\begin{array}{l}\text { Cellular metabolic } \\
\text { process }\end{array}$ & Mucus CRS & 0.00199 & LDHA;TPI1;PKM;ALDOA \\
\hline
\end{tabular}




\begin{tabular}{|c|c|c|c|c|}
\hline Biological Process & Classification & Source & $\begin{array}{l}\text { Adjusted } \\
\text { P-value }\end{array}$ & Genes \\
\hline $\begin{array}{l}\text { glycolytic process through glucose-6-phosp- } \\
\text { hate (GO:0061620) }\end{array}$ & $\begin{array}{l}\text { Cellular metabolic } \\
\text { process }\end{array}$ & Mucus CRS & 0.00016 & TPI1;PKM;ENO1;ALDOA;GAPDH \\
\hline heart contraction (GO:0060047) & Circulatory system & Mucosa Healthy + CRS & 0.04000 & MYL4;TPM1;SOD1 \\
\hline hexose biosynthetic process (GO:0019319) & Metabolic process & Mucus CRS & 0.02409 & TPI1;ENO1;ALDOA;GAPDH \\
\hline homotypic cell-cell adhesion (GO:0034109) & Cell-cell adhesion & Mucosa Healthy + CRS & 0.03709 & FGG;ACTB;ACTG1 \\
\hline $\begin{array}{l}\text { humoral immune response mediated by circu- } \\
\text { lating immunoglobulin (GO:0002455) }\end{array}$ & $\begin{array}{l}\text { Immune system } \\
\text { process }\end{array}$ & Mucus CRS & 0.00004 & $\begin{array}{l}\text { IGHG3;IGHG4;IGHM;IGHG1;IGHG2;IG } \\
\text { KC;IGHV3-23;IGLC1;IGHA2 }\end{array}$ \\
\hline $\begin{array}{l}\text { hydrogen peroxide metabolic process } \\
\text { (GO:0042743) }\end{array}$ & $\begin{array}{l}\text { Cellular metabolic } \\
\text { process }\end{array}$ & Mucosa Healthy + CRS & 0.00024 & PRDX2;CAT;PARK7;SOD1 \\
\hline $\begin{array}{l}\text { intermediate filament organization } \\
\text { (GO:0045109) }\end{array}$ & $\begin{array}{l}\text { Cellular compo- } \\
\text { nent organisation }\end{array}$ & Mucus CRS & 0.00001 & DSP;KRT14;PKP1;KRT20;KRT9 \\
\hline keratinocyte differentiation (GO:0030216) & $\begin{array}{l}\text { System develop- } \\
\text { ment }\end{array}$ & Mucus CRS & 0.00000 & $\begin{array}{l}\text { DSP;FLG;CSTA;ANXA1;KRT16;KRT10 } \\
\text {;SPRR2B;SPRR1A;TGM3;IVL;SPRR1B; } \\
\text { S100A7 }\end{array}$ \\
\hline $\begin{array}{l}\text { negative regulation of apoptotic process } \\
\text { (GO:0043066) }\end{array}$ & Cell death & Mucosa CRS & 0.02026 & $\begin{array}{l}\text { HSPA9;FABP1;HSPA5;GSTP1;GLO1; } \\
\text { HSPD1 }\end{array}$ \\
\hline $\begin{array}{l}\text { negative regulation of apoptotic process } \\
\text { (GO:0043066) }\end{array}$ & Cell death & Mucosa Healthy + CRS & 0.00048 & $\begin{array}{l}\text { PRDX2;ANXA1;ARHGDIA;CAT;PARK7; } \\
\text { TPT1;HSP90B1;TXNDC5;SOD1 }\end{array}$ \\
\hline $\begin{array}{l}\text { negative regulation of cellular protein meta- } \\
\text { bolic process (GO:0032269) }\end{array}$ & $\begin{array}{l}\text { Cellular metabolic } \\
\text { process }\end{array}$ & Mucus CRS & 0.03466 & SERPINB3;CSTB;CSTA;GAPDH;CST4 \\
\hline $\begin{array}{l}\text { negative regulation of endopeptidase activity } \\
\text { (GO:0010951) }\end{array}$ & $\begin{array}{l}\text { Regulation of mo- } \\
\text { lecular function }\end{array}$ & Mucus CRS & 0.00003 & $\begin{array}{l}\text { SERPINB3;SERPINA3;SERPINB4;SERPI } \\
\text { NB12;SERPINA1;AHSG;GAPDH;CST4 }\end{array}$ \\
\hline $\begin{array}{l}\text { negative regulation of peptidase activity } \\
\text { (GO:0010466) }\end{array}$ & $\begin{array}{l}\text { Regulation of mo- } \\
\text { lecular function }\end{array}$ & Mucus CRS & 0.00000 & $\begin{array}{l}\text { SERPINB3;SERPINA3;SERPINB4;CST } \\
\text { B;SERPINB12;CSTA;SERPINA1;AHSG } \\
\text {;GAPDH }\end{array}$ \\
\hline $\begin{array}{l}\text { negative regulation of programmed cell death } \\
\text { (GO:0043069) }\end{array}$ & Cell death & Mucosa CRS & 0.01505 & $\begin{array}{l}\text { HSPA9;FABP1;HSPA5;GSTP1;GLO1; } \\
\text { HSPD1 }\end{array}$ \\
\hline $\begin{array}{l}\text { negative regulation of programmed cell death } \\
\text { (GO:0043069) }\end{array}$ & Cell death & Mucosa Healthy + CRS & 0.00112 & $\begin{array}{l}\text { PRDX2;ANXA1;ARHGDIA;CAT;PARK7; } \\
\text { TPT1;HSP90B1;TXNDC5 }\end{array}$ \\
\hline $\begin{array}{l}\text { negative regulation of proteolysis } \\
\text { (GO:0045861) }\end{array}$ & $\begin{array}{l}\text { Cellular metabolic } \\
\text { process }\end{array}$ & Mucus CRS & 0.00133 & SERPINB3;CSTB;SERPINB4;CSTA;CST4 \\
\hline $\begin{array}{l}\text { neutrophil activation involved in immune } \\
\text { response (GO:0002283) }\end{array}$ & $\begin{array}{l}\text { Immune system } \\
\text { process }\end{array}$ & Mucus CRS & 0.00000 & $\begin{array}{l}\text { DSP;SERPINB3;SERPINA3;PIGR;CSTB;S } \\
\text { ERPINB12;SERPINA1;CALML5;JUP;AN } \\
\text { XA2;AHSG;GSTP1;DEFA1;HRNR;EEF1 } \\
\text { A1;PKM;FABP5;PKP1;DSG1;ALDOA;B2 } \\
\text { M;S100A8;S100A11;S100A7 }\end{array}$ \\
\hline $\begin{array}{l}\text { neutrophil activation involved in immune } \\
\text { response (GO:0002283) }\end{array}$ & $\begin{array}{l}\text { Immune system } \\
\text { process }\end{array}$ & Mucus Healthy + CRS & 0.00069 & ORM1;SLPI;HBB;LYZ;S100A9;LTF \\
\hline neutrophil degranulation (GO:0043312) & $\begin{array}{l}\text { Immune system } \\
\text { process }\end{array}$ & Mucus CRS & 0.00000 & $\begin{array}{l}\text { DSP;SERPINB3;SERPINA3;PIGR;CSTB;S } \\
\text { ERPINB12;SERPINA1;CALML5;JUP;AN } \\
\text { XA2;AHSG;GSTP1;DEFA1;HRNR;EEF1 } \\
\text { A1;PKM;FABP5;PKP1;DSG1;ALDOA;B2 } \\
\text { M;S100A8;S100A11;S100A7 }\end{array}$ \\
\hline neutrophil degranulation (GO:0043312) & $\begin{array}{l}\text { Immune system } \\
\text { process }\end{array}$ & Mucus Healthy + CRS & 0.00079 & ORM1;SLPI;HBB;LYZ;S100A9;LTF \\
\hline neutrophil mediated immunity (GO:0002446) & $\begin{array}{l}\text { Immune system } \\
\text { process }\end{array}$ & Mucus CRS & 0.00000 & $\begin{array}{l}\text { DSP;SERPINB3;SERPINA3;PIGR;CSTB;S } \\
\text { ERPINB12;SERPINA1;CALML5;JUP;AN } \\
\text { XA2;AHSG;GSTP1;DEFA1;HRNR;EEF1 } \\
\text { A1;PKM;FABP5;PKP1;DSG1;ALDOA;B2 } \\
\text { M;S100A8;S100A11;S100A7 }\end{array}$ \\
\hline neutrophil mediated immunity (GO:0002446) & $\begin{array}{l}\text { Immune system } \\
\text { process }\end{array}$ & Mucus Healthy + CRS & 0.00062 & ORM1;SLPI;HBB;LYZ;S100A9;LTF \\
\hline $\begin{array}{l}\text { nicotinamide nucleotide metabolic process } \\
\text { (GO:0046496) }\end{array}$ & $\begin{array}{l}\text { Cellular metabolic } \\
\text { process }\end{array}$ & Mucus CRS & 0.02575 & LDHA;TPI1;PKM;ALDOA \\
\hline $\begin{array}{l}\text { pattern recognition receptor signaling pa- } \\
\text { thway (GO:0002221) }\end{array}$ & $\begin{array}{l}\text { Immune system } \\
\text { process }\end{array}$ & Mucosa Healthy + CRS & 0.03975 & FGG;S100A8;HSP90B1 \\
\hline
\end{tabular}




\begin{tabular}{|c|c|c|c|c|}
\hline Biological Process & Classification & Source & $\begin{array}{l}\text { Adjusted } \\
\text { P-value }\end{array}$ & Genes \\
\hline peptide cross-linking (GO:0018149) & $\begin{array}{l}\text { Cellular metabolic } \\
\text { process }\end{array}$ & Mucus CRS & 0.00000 & $\begin{array}{l}\text { FLG;DSP;CSTA;ANXA1;KRT2;KRT10;SP } \\
\text { RR2B;SPRR1A;TGM3;SPRR1B;IVL }\end{array}$ \\
\hline peptidyl-cysteine modification (GO:0018198) & $\begin{array}{l}\text { Cellular metabolic } \\
\text { process }\end{array}$ & Mucosa Healthy + CRS & 0.03937 & PARK7;S100A8 \\
\hline phagocytosis (GO:0006909) & Localisation & Mucus CRS & 0.00000 & $\begin{array}{l}\text { IGHG3;IGHG4;IGHM;IGHG1; } \\
\text { IGHG2;ANXA1;IGKC;IGHV3- } \\
\text { 23;MYH9;IGLC1;IGHA2 }\end{array}$ \\
\hline phagocytosis, engulfment (GO:0006911) & Localisation & Mucus CRS & 0.00000 & $\begin{array}{l}\text { IGHG3;IGHG4;IGHM;IGHG1;IGHG2;IG } \\
\text { KC;IGHV3-23;MYH9;IGLC1;IGHA2 }\end{array}$ \\
\hline plasma membrane invagination (GO:0099024) & $\begin{array}{l}\text { Cellular compo- } \\
\text { nent organisation }\end{array}$ & Mucus CRS & 0.00000 & $\begin{array}{l}\text { IGHG3;IGHG4;IGHM;IGHG1;IGHG2;IG } \\
\text { KC;IGHV3-23;MYH9;IGLC1;IGHA2 }\end{array}$ \\
\hline platelet aggregation (GO:0070527) & $\begin{array}{l}\text { Reponse to } \\
\text { stimulus }\end{array}$ & Mucosa Healthy + CRS & 0.02851 & FGG;ACTB;ACTG1 \\
\hline platelet degranulation (GO:0002576) & Export from cell & Mucus Healthy + CRS & 0.03274 & ORM1;TF;ALB \\
\hline platelet degranulation (GO:0002576) & Export from cell & Mucosa Healthy + CRS & 0.03797 & SERPINA1;FGG;APOA1;SOD1 \\
\hline $\begin{array}{l}\text { positive regulation of B cell activation } \\
\text { (GO:0050871) }\end{array}$ & Cell activation & Mucus CRS & 0.00003 & $\begin{array}{l}\text { IGHG3;IGHG4;IGHM;IGHG1;IGHG2;IG } \\
\text { KC;IGHV3-23;IGLC1;IGHA2 }\end{array}$ \\
\hline $\begin{array}{l}\text { positive regulation of lymphocyte activation } \\
\text { (GO:0051251) }\end{array}$ & Cell activation & Mucus CRS & 0.00001 & $\begin{array}{l}\text { IGHG3;IGHG4;IGHM;IGHG1;IGHG2;IG } \\
\text { KC;IGHV3-23;IGLC1;IGHA2 }\end{array}$ \\
\hline $\begin{array}{l}\text { positive regulation of metabolic process } \\
\text { (GO:0009893) }\end{array}$ & Metabolic process & Mucus Healthy + CRS & 0.02714 & IGHA1;JCHAIN \\
\hline $\begin{array}{l}\text { positive regulation of respiratory burst } \\
\text { (GO:0060267) }\end{array}$ & Metabolic process & Mucus Healthy + CRS & 0.00831 & IGHA1;JCHAIN \\
\hline protein heterooligomerization (GO:0051291) & $\begin{array}{l}\text { Cellular compo- } \\
\text { nent organisation } \\
\text { or biogenesis }\end{array}$ & Mucus CRS & 0.00266 & $\begin{array}{l}\text { HIST1H4A;ANXA2;SEMG2;SEMG1; } \\
\text { HBA1 }\end{array}$ \\
\hline pyruvate metabolic process (GO:0006090) & $\begin{array}{l}\text { Cellular metabolic } \\
\text { process }\end{array}$ & Mucus CRS & 0.02748 & LDHA;TPI1;PKM;ALDOA \\
\hline receptor-mediated endocytosis (G0:0006898) & Localisation & Mucus Healthy + CRS & 0.00025 & IGKV1-5;ALB;HBB;IGHA1;JCHAIN \\
\hline $\begin{array}{l}\text { regulation of acute inflammatory response } \\
\text { (GO:0002673) }\end{array}$ & $\begin{array}{l}\text { Reponse to } \\
\text { stimulus }\end{array}$ & Mucus CRS & 0.00033 & $\begin{array}{l}\text { IGHG3;IGHG4;IGHG 1;IGHG2;IGKC; } \\
\text { GSTP1; } \\
\text { IGHV3-23;IGLC1 }\end{array}$ \\
\hline regulation of apoptotic process (GO:0042981) & Cell death & Mucosa CRS & 0.01647 & $\begin{array}{l}\text { HSPA9;FABP1;HSPA5;GSTP1;GLO1;TA } \\
\text { OK1;HSPD1 }\end{array}$ \\
\hline regulation of apoptotic process (GO:0042981) & Cell death & Mucosa Healthy + CRS & 0.00197 & $\begin{array}{l}\text { PRDX2;ANXA1;ARHGDIA;CAT;NME4;P } \\
\text { ARK7;TPT1;HSP90B1;TXNDC5;SOD1 }\end{array}$ \\
\hline regulation of B cell activation (GO:0050864) & Cell activation & Mucus CRS & 0.00001 & $\begin{array}{l}\text { IGHG3;IGHM;IGHG4;IGHG 1;IGHG2;IG } \\
\text { KC;IGHV3-23;IGLC1;IGHA2 }\end{array}$ \\
\hline $\begin{array}{l}\text { regulation of complement activation } \\
\text { (GO:0030449) }\end{array}$ & $\begin{array}{l}\text { Immune system } \\
\text { process }\end{array}$ & Mucus CRS & 0.00137 & $\begin{array}{l}\text { IGHG3;IGHG4;IGHG1;IGHG2;IGKC;IG } \\
\text { HV3-23;IGLC1 }\end{array}$ \\
\hline $\begin{array}{l}\text { regulation of endopeptidase activity } \\
\text { (GO:0052548) }\end{array}$ & $\begin{array}{l}\text { Regulation of mo- } \\
\text { lecular function }\end{array}$ & Mucus CRS & 0.00009 & $\begin{array}{l}\text { SERPINB3;SERPINA3;SERPINB4;SERPI } \\
\text { NB12;SERPINA1;AHSG;GAPDH }\end{array}$ \\
\hline $\begin{array}{l}\text { regulation of humoral immune response } \\
\text { (GO:0002920) }\end{array}$ & $\begin{array}{l}\text { Immune system } \\
\text { process }\end{array}$ & Mucus CRS & 0.00165 & $\begin{array}{l}\text { IGHG3;IGHG4;IGHG1;IGHG2;IGKC;IG } \\
\text { HV3-23;IGLC1 }\end{array}$ \\
\hline $\begin{array}{l}\text { regulation of immune effector process } \\
\text { (GO:0002697) }\end{array}$ & $\begin{array}{l}\text { Immune system } \\
\text { process }\end{array}$ & Mucus CRS & 0.00170 & $\begin{array}{l}\text { IGHG3;IGHG4;IGHG1;IGHG2;IGKC;IG } \\
\text { HV3-23;IGLC1 }\end{array}$ \\
\hline regulation of peptidase activity (GO:0052547) & $\begin{array}{l}\text { Regulation of mo- } \\
\text { lecular function }\end{array}$ & Mucus CRS & 0.00085 & SERPINB3;SERPINB4;CSTB;CSTA \\
\hline $\begin{array}{l}\text { regulation of protein activation cascade } \\
\text { (GO:2000257) }\end{array}$ & Metabolic process & Mucus CRS & 0.00133 & $\begin{array}{l}\text { IGHG3;IGHG4;IGHG1;IGHG2;IGKC;IG } \\
\text { HV3-23;IGLC1 }\end{array}$ \\
\hline regulation of protein processing (GO:0070613) & Metabolic process & Mucus CRS & 0.00327 & $\begin{array}{l}\text { IGHG3;IGHG4;IGHG1;IGHG2;IGKC;IG } \\
\text { HV3-23;IGLC1 }\end{array}$ \\
\hline regulation of proteolysis (GO:0030162) & Metabolic process & Mucus CRS & 0.00828 & SERPINB3;SERPINB4;CSTB;CSTA;CST4 \\
\hline regulation of respiratory burst (GO:0060263) & Metabolic process & Mucus Healthy + CRS & 0.01001 & IGHA1;JCHAIN \\
\hline
\end{tabular}




\begin{tabular}{|c|c|c|c|c|}
\hline Biological Process & Classification & Source & $\begin{array}{l}\text { Adjusted } \\
\text { P-value }\end{array}$ & Genes \\
\hline regulation of water loss via skin (GO:0033561) & $\begin{array}{l}\text { Biological regu- } \\
\text { lation }\end{array}$ & Mucus CRS & 0.00058 & FLG;KRT16;SFN;HRNR \\
\hline removal of superoxide radicals (GO:0019430) & $\begin{array}{l}\text { Cellular reponse to } \\
\text { chemical stimulus }\end{array}$ & Mucosa Healthy + CRS & 0.04334 & PRDX2;SOD1 \\
\hline renal filtration (GO:0097205) & System process & Mucus Healthy + CRS & 0.01129 & IGHA1;JCHAIN \\
\hline response to hydrogen peroxide (GO:0042542) & $\begin{array}{l}\text { Reponse to } \\
\text { stimulus }\end{array}$ & Mucosa Healthy + CRS & 0.04154 & CAT;PARK7;SOD1 \\
\hline response to unfolded protein (GO:0006986) & $\begin{array}{l}\text { Reponse to } \\
\text { stimulus }\end{array}$ & Mucosa CRS & 0.02186 & HSPA9;HSPA5;HSPD1 \\
\hline retina homeostasis (GO:0001895) & $\begin{array}{l}\text { Tissue homeo- } \\
\text { stasis }\end{array}$ & Mucus CRS & 0.00000 & $\begin{array}{l}\text { IGHG3;PIGR;AZGP1;IGKC;PIP;HSPB1 } \\
\text { LCN1;B2M;IGHA2;ACTB;CST4 }\end{array}$ \\
\hline retina homeostasis (GO:0001895) & $\begin{array}{l}\text { Tissue homeo- } \\
\text { stasis }\end{array}$ & Mucus Healthy + CRS & 0.00000 & TF;ALB;LYZ;IGHA1;JCHAIN;LTF \\
\hline retina homeostasis (GO:0001895) & $\begin{array}{l}\text { Tissue homeo- } \\
\text { stasis }\end{array}$ & Mucosa Healthy + CRS & 0.03245 & ACTB;ACTG1;SOD1 \\
\hline $\begin{array}{l}\text { sensory perception of bitter taste } \\
\text { (GO:0050913) }\end{array}$ & $\begin{array}{l}\text { Sensory percep- } \\
\text { tion }\end{array}$ & Mucus CRS & 0.01951 & PIGR;AZGP1;PIP;CST4 \\
\hline skin development (GO:0043588) & $\begin{array}{l}\text { System develop- } \\
\text { ment }\end{array}$ & Mucus CRS & 0.00000 & $\begin{array}{l}\text { DSP;FLG;CSTA;ANXA1;KRT10;ASPRV } \\
\text { 1;KRT9;HRNR;COL1A1;COL1A2;KRT1 } \\
\text { 6;SFN;SPRR2B;SPRR1A;TGM3;IVL;SPR } \\
\text { R1B;S100A7 }\end{array}$ \\
\hline
\end{tabular}

\section{Molecular function}

\begin{tabular}{|c|c|c|c|c|}
\hline Molecular Function & Classification & Group & $\begin{array}{l}\text { Adjusted } \\
\text { P-value }\end{array}$ & Genes \\
\hline cadherin binding (GO:0045296) & Binding & Mucus CRS & 0.00294 & $\begin{array}{l}\text { LDHA;ANXA1;PKM;JUP;ANXA2;MYH9 } \\
\text {;SFN;ENO1;ALDOA;YWHAZ;S100A11 }\end{array}$ \\
\hline $\begin{array}{l}\text { cadherin binding involved in cell-cell adhe- } \\
\text { sion (GO:0098641) }\end{array}$ & Binding & Mucus CRS & 0.03519 & ANXA1;ANXA2;S100A11 \\
\hline $\begin{array}{l}\text { cysteine-type endopeptidase inhibitor activity } \\
\text { (GO:0004869) }\end{array}$ & $\begin{array}{l}\text { Molecular function } \\
\text { regulator }\end{array}$ & Mucus CRS & 0.00625 & SERPINB3;CSTB;CSTA;LCN1;CST4 \\
\hline endopeptidase activity (GO:0004175) & Catalytic activity & Mucus CRS & 0.02312 & $\begin{array}{l}\text { IGHG3;IGHG4;CASP14;IGHG1;IGHG2;I } \\
\text { GKC;PIP;IGHV3-23;IGLC1;ASPRV1 }\end{array}$ \\
\hline endopeptidase inhibitor activity (GO:0004866) & $\begin{array}{l}\text { Molecular function } \\
\text { regulator }\end{array}$ & Mucus CRS & 0.00000 & $\begin{array}{l}\text { SERPINB3;SERPINA3;SERPINB4;CSTB; } \\
\text { SERPINB12;CSTA;SERPINA1;AHSG;LC } \\
\text { N1;GAPDH;CST4 }\end{array}$ \\
\hline $\begin{array}{l}\text { immunoglobulin receptor binding } \\
\text { (GO:0034987) }\end{array}$ & Binding & Mucus CRS & 0.00000 & $\begin{array}{l}\text { IGHG3;IGHM;IGHG4;IGHG1;IGHG2;IG } \\
\text { KC;IGHV3-23;IGLC1;IGHA2 }\end{array}$ \\
\hline intermediate filament binding (GO:0019215) & Binding & Mucus CRS & 0.00802 & KRT14;PKP1;VIM \\
\hline protease binding (GO:0002020) & Binding & Mucus CRS & 0.00000 & $\begin{array}{l}\text { COL1A1;SERPINB3;SERPINB4;CSTB;C } \\
\text { STA;COL1A2;SERPINA1;ANXA2;SEMG } \\
\text { 2;UBC;CST4 }\end{array}$ \\
\hline $\begin{array}{l}\text { protein binding involved in cell-cell adhesion } \\
\text { (GO:0098632) }\end{array}$ & Binding & Mucus CRS & 0.04508 & ANXA1;ANXA2;S100A11 \\
\hline $\begin{array}{l}\text { serine-type endopeptidase activity } \\
\text { (GO:0004252) }\end{array}$ & Catalytic activity & Mucus CRS & 0.04657 & $\begin{array}{l}\text { IGHG3;IGHG4;IGHG1;IGHG2;IGKC;IG } \\
\text { HV3-23;IGLC1 }\end{array}$ \\
\hline $\begin{array}{l}\text { serine-type endopeptidase inhibitor activity } \\
\text { (GO:0004867) }\end{array}$ & $\begin{array}{l}\text { Molecular function } \\
\text { regulator }\end{array}$ & Mucus CRS & 0.00817 & $\begin{array}{l}\text { SERPINB3;SERPINA3;SERPINB4;SERPI } \\
\text { NB12;SERPINA1 }\end{array}$ \\
\hline $\begin{array}{l}\text { signal recognition particle binding } \\
\text { (GO:0005047) }\end{array}$ & Binding & Mucus Healthy + CRS & 0.01688 & TF;LTF \\
\hline
\end{tabular}

